\title{
Natural supersymmetry and unification in five dimensions
}

\author{
Ammar Abdalgabar, ${ }^{a, b}$ Alan S. Cornell, ${ }^{a}$ Aldo Deandrea ${ }^{c, d, e}$ and Moritz McGarrie ${ }^{f}$ \\ ${ }^{a}$ National Institute for Theoretical Physics and School of Physics and \\ Mandelstam Institute for Theoretical Physics, University of the Witwatersrand, \\ Private Bag 3, Wits, 2050 South Africa \\ ${ }^{b}$ Department of Physics, Sudan University of Science and Technology, \\ Khartoum, 407 Sudan \\ ${ }^{c}$ Université de Lyon, \\ 92, rue Pasteur, Lyon, F-69361 France \\ ${ }^{d} I P N L$, Université Lyon 1, CNRS/IN2P3, \\ 4 rue Fermi, Villeurbanne Cedex, F-69622 France \\ ${ }^{e}$ Institut Universitaire de France, \\ 103 boulevard Saint-Michel, Paris, 75005 France \\ ${ }^{f}$ Faculty of Physics, University of Warsaw, \\ Hoża 69, Warsaw, 00-681 Poland \\ E-mail: ammar.abdalgabar@wits.ac.za, alan.cornell@wits.ac.za, \\ deandrea@ipnl.in2p3.fr, moritz.mcgarrie@fuw.edu.pl
}

ABSTRACT: We explore unification and natural supersymmetry in a five dimensional extension of the standard model in which the extra dimension may be large, of the order of $1-10 \mathrm{TeV}$. Power law running generates a TeV scale $A_{t}$ term allowing for the observed $125 \mathrm{GeV}$ Higgs and allowing for stop masses below $2 \mathrm{TeV}$, compatible with a natural SUSY spectrum. We supply the full one-loop RGEs for various models and use metastability to give a prediction that the gluino mass should be lighter than $3.5 \mathrm{TeV}$ for $A_{t} \geq-2.5 \mathrm{TeV}$, for such a compactification scale, with brane localised 3rd generation matter. We also discuss models in which only the 1st and 2nd generation of matter fields are located in the bulk. We also look at electroweak symmetry breaking in these models.

Keywords: Beyond Standard Model, Higgs Physics, Supersymmetric Standard Model

ARXiv EPrint: 1504.07749 


\section{Contents}

1 Introduction 1

2 5D-SSM with additional states: unification 2

2.1 Gauge coupling unification 3

3 Exploring the models $\quad 5$

3.1 Typical scales of the models 6

$\begin{array}{lll}3.2 & \text { Implementation and results } & 7\end{array}$

3.3 Two ways to accommodate natural supersymmetry 9

$\begin{array}{ll}\text { 3.3.1 The third generation in the bulk } & 10\end{array}$

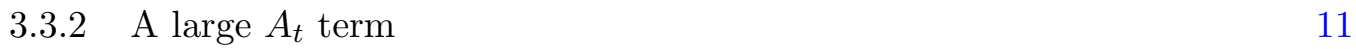

4 The Higgs mass 11

5 Conclusions 13

A Renormalisation group equations for $4 \mathrm{D}-\mathrm{SSM}+\boldsymbol{F}^{ \pm} \quad 14$

$\begin{array}{lll}\text { A.1 Anomalous dimensions } & 14\end{array}$

$\begin{array}{ll}\text { A.2 Gauge couplings } & 15\end{array}$

$\begin{array}{lll}\text { A.3 Gaugino mass parameters } & 15\end{array}$

$\begin{array}{lll}\text { A.4 Trilinear superpotential parameters } & 16\end{array}$

$\begin{array}{lll}\text { A.5 Bilinear superpotential parameters } & 17\end{array}$

$\begin{array}{ll}\text { A.6 Trilinear soft-breaking parameters } & 17\end{array}$

$\begin{array}{ll}\text { A.7 Vacuum expectation values } & 19\end{array}$

$\begin{array}{lll}\text { A.8 Bilinear soft-breaking parameters } & 19\end{array}$

$\begin{array}{lll}\text { A.9 } & \text { Soft-breaking scalar masses } & 20\end{array}$

B Renormalisation group equations for the 5D-SSM+F $F^{ \pm} \quad \mathbf{2 5}$

$\begin{array}{ll}\text { B.1 Gauge couplings } & 25\end{array}$

B.2 Yukawa couplings 25

B.2.1 Anomalous dimensions for model $1 \quad 26$

B.2.2 Anomalous dimensions for model $2 \quad 26$

$\begin{array}{ll}\text { B.2.3 Anomalous dimensions for model } 3 & 27\end{array}$

$\begin{array}{lll}\text { B.2.4 Yukawa coupling RGEs for model } 1 & 27\end{array}$

B.2.5 Yukawa coupling RGEs for model $2 \quad 27$

B.2.6 Yukawa coupling RGEs for model $3 \quad 28$

$\begin{array}{lll}\text { B.3 Trilinear soft breaking parameters } & 28\end{array}$

B.3.1 Trilinear soft breaking parameters for model $1 \quad 28$

B.3.2 Trilinear soft breaking parameters for model $2 \quad 29$

$\begin{array}{lll}\text { B.4 Soft mass parameters } & 29\end{array}$

$\begin{array}{ll}\text { B.4.1 Gaugino soft mass parameters } & 29\end{array}$ 
B.4.2 Scalar soft mass parameters for model 1

B.4.3 Scalar soft mass parameters for model 2

\section{Introduction}

In the context of supersymmetry and through the prism of the naturalness aesthetic, the discovery of a Standard Model-like scalar particle of mass $m_{h} \sim 125 \mathrm{GeV}[1,2]$, and no direct evidence so far of superparticles has motivated renewed interest in non-minimal extensions of the Supersymmetric Standard Model (SSM) that can help to compellingly explain such results. Within the Minimal-SSM (MSSM), for the lightest CP even charge neutral scalar to be the discovered scalar then requires either multi-TeV stops, which is disfavoured from naturalness, an enhancement to the tree-level Higgs mass such as for example [3-6], or a near maximal mixing scenario whereby $\left|A_{t}\left(M_{z}\right)\right| \gtrsim 1 \mathrm{TeV}$. There are few models that compellingly achieve a large enough $A_{t}$ if one first assumes $A_{t}$ to vanish at some initial supersymmetry breaking scale. Even if one obtains such a large $A_{t}$, one must still explain why stops are lighter than their first and second generation counterpart squarks, consistent with collider bounds [7-17]. One such framework that can address both problems is a five dimensional-SSM.

In five dimensional (5D) SSMs, power law running for a sufficiently low compactification radius $R$, generates at low energies a large enough $A_{t}$ to explain the observed Higgs mass [18]. Furthermore, through spatially localising different generations along the extra dimension(s), one can explain geometrically why the third generation can be consistently lighter than its first and second generation counterparts [18].

This framework is sufficiently compelling that it should understandably endure further scrutiny. In particular, five dimensional theories are effective field theories with a cutoff and are (often over-dramatically) defined as non-renormalisable, as many parameters such as gauge couplings can be sensitive to this UV scale. It is therefore important to confirm that results and conclusions made at one loop that are sensitive to this scale are still consistent and under control at two (and higher) loops. For instance one might be concerned that one loop linear sensitivity to the cutoff behaving as $\Lambda R$ do not result in terms of the form $(\Lambda R)^{2}$ at two-loop, which would then indicate a break-down of perturbation theory at renormalisation scales of the order of the compactification radius [19]. Whilst this might be of concern to non-supersymmetric theories, the five dimensional SSM is reinterpreted in the language of $\mathcal{N}=2$ four dimensional supersymmetry. This additional supersymmetry and the protection it affords, helps to reduce such terms [20,21], at least for gauge couplings. The effect remains but has opposite sign for both Yukawa couplings and their soft breaking trilinear counterparts, and so is still under complete control. For the case of bulk matter and in particular the top Yukawa in the bulk, a Landau pole appears and one must then seriously consider that either perturbation theory is problematic for these models (just 
as one would in any four dimensional theory with a Landau pole), or that a compelling explanation of how the top Yukawa may arise must be found such that this pathology may be avoided. Another issue which is a general one in these effective theories, is that in order to be sure that no unwanted operators are generated one should consider the possible UV completions. This point is discussed in the literature, and is typically a difficult model building effort, but goes beyond the scope of the present work which is limited to the investigation of the effective theory. Furthermore, possible issues related to proton decay shall be briefly mentioned in section 2.1 .

There are further criteria for our model to be truly compelling: we require that it is supersymmetric and that supersymmetry is softly broken, that the superpotential is renormalisable and that the theory's gauge couplings unify in the five dimensional description with a large enough extra dimensional scale as to make the extra dimensional features practically relevant to the phenomenology of the model. In other words we require a $1 / R \sim 1$ to $10^{3} \mathrm{TeV}$ scale extra dimension and not simply an (almost) GUT scale extra dimension. Such a criteria is useful to rule out certain models, for instance by this criteria one can straightforwardly rule out flat extra dimensional models in which the 1st and 2nd generation are in the bulk, with the 3rd generation either in the bulk or on a brane, as such a model can only unify with an extra dimensional scale of the order of the GUT scale, a topic we discuss in more detail later.

The outline of the paper is as follows: in section 2 we describe the models in detail and discuss unification. In section 3 we describe our boundary conditions and how the four dimensional (4D) and 5D renormalisation group equations (RGEs) are matched and solved. We discuss the various energy scales of the model and then look at the running of various parameters including the gaugino mass spectrum and trilinear soft breaking terms. In section 4 we explore how to obtain the correct $125 \mathrm{GeV}$ Higgs mass, with stops lighter than $2 \mathrm{TeV}$. In section 5 we give our conclusions. We also include two detailed appendices, appendix A including all the one-loop and two-loop RGEs of the four dimensional low energy model, of which we used the one-loop RGEs in the plots, and appendix B includes the one-loop RGEs for the five dimensional models 1 and 2 of the main paper. The conventions and notation of this paper follow closely that of [18], which are based on conventions found in [22-26].

\section{5D-SSM with additional states: unification}

A TeV scale SSM in which the gauge coupling is precisely unified is proposed in [27]. The key idea is to add two new hypermultiplets $F^{ \pm}$which are singlets under $\mathrm{SU}(3)_{c} \times \mathrm{SU}(2)_{L}$ and charged under $\mathrm{U}(1)_{Y}$ with $Y_{F^{ \pm}}= \pm 1$. The SSM chiral fermions are located on a boundary and in the 5D picture do not have Kaluza-Klein (KK) modes. The SSM Higgs chiral multiplets live in the bulk and we embed them as hypermultiplets in 5D. The gauge fields and the additional states also live in the bulk as listed in table 1: we call this model 1. We will also explore our own model in which the third generation of superfields lives in the bulk, as in table 2: we call this model 2 and this too may unify. We compute and collate all supersymmetric and soft-term RGEs. These new states modify the beta 


\begin{tabular}{|c|c|c|c|}
\hline Superfields & Brane & Bulk & $\mathrm{U}(1)_{Y} \times \mathrm{SU}(2)_{L} \times \mathrm{SU}(3)_{c}$ \\
\hline$\hat{q}^{f}$ & $\checkmark$ & - & $\left(\frac{1}{6}, \mathbf{2}, \mathbf{3}\right)$ \\
$\hat{d}^{f}$ & $\checkmark$ & - & $\left(\frac{1}{3}, \mathbf{1}, \overline{\mathbf{3}}\right)$ \\
$\hat{u}^{f}$ & $\checkmark$ & - & $\left(-\frac{2}{3}, \mathbf{1}, \overline{\mathbf{3}}\right)$ \\
$\hat{l}^{f}$ & $\checkmark$ & - & $\left(-\frac{1}{2}, \mathbf{2}, \mathbf{1}\right)$ \\
$\hat{e}^{f}$ & $\checkmark$ & - & $(1, \mathbf{1}, \mathbf{1})$ \\
\hline$\hat{H}_{d}$ & - & $\checkmark$ & $\left(-\frac{1}{2}, \mathbf{2}, \mathbf{1}\right)$ \\
$\hat{H}_{u}$ & - & $\checkmark$ & $\left(\frac{1}{2}, \mathbf{2}, \mathbf{1}\right)$ \\
\hline$\hat{F}_{-}$ & - & $\checkmark$ & $(-1, \mathbf{1}, \mathbf{1})$ \\
$\hat{F}_{+}$ & - & $\checkmark$ & $(1, \mathbf{1}, \mathbf{1})$ \\
\hline$\hat{B}_{V}$ & - & $\checkmark$ & $(0, \mathbf{1}, \mathbf{1})$ \\
$\hat{W}_{V}$ & - & $\checkmark$ & $(0, \mathbf{3}, \mathbf{1})$ \\
$\hat{G}_{V}$ & - & $\checkmark$ & $(0, \mathbf{1}, \mathbf{8})$ \\
\hline
\end{tabular}

Table 1. The matter content of model 1. All superfields of chiral fermions live on a brane and all Higgs-type superfields and gauge vector fields live in the bulk. The superscript $f=1,2,3$ denotes the generations. Neutrino superfields may be included straightforwardly. The gauge couplings of this model unify as in figure 1 (top left).

function coefficient $b_{1}$ and lead to precision unification at one-loop. The superpotential for both models is given by

$$
W=Y_{u} \hat{u} \epsilon_{i j} \hat{q}^{i} \hat{H}_{u}^{j}-Y_{d} \hat{d} \epsilon_{i j} \hat{q}^{i} \hat{H}_{d}^{j}-Y_{e} \hat{e} \epsilon_{i j} \hat{l}^{i} \hat{H}_{d}^{j}+\mu H_{u} H_{d}+\mu F^{-} F^{+} .
$$

\subsection{Gauge coupling unification}

A sufficient condition for unification in a five dimensional model is $[28,29]$ that

$$
R_{i j}=\frac{b_{i}^{(5 D)}-b_{j}^{(5 D)}}{b_{i}^{\mathrm{SSM}}-b_{j}^{\mathrm{SSM}}}
$$

does not depend on $(i, j)$, where $b_{i}^{5 D}$ are the five dimensional beta function coefficients, at one-loop. The $\beta$-function of an $\mathrm{SU}(N)$ gauge theory at one-loop is

$$
\beta_{g}=\mu \frac{d g}{d \mu}=-\frac{g^{3}}{16 \pi^{2}}\left(\frac{11}{3} T(A d j)-\frac{2}{3} T_{\text {fer }}(R)-\frac{1}{3} T_{s c}(R)\right)=\frac{b_{g}^{(1-\text { loop })} g^{3}}{(2 \pi)^{4}}
$$

for gauge fields, Weyl fermions and complex scalars respectively. $R$ is the representation and in particular $T(\mathrm{Ad})=N$ and $T(\square)=\frac{1}{2}$.

For a $\mathrm{U}(1)$ theory [30] the gauge field is uncharged, there is also an overall normalisation constant which can be fixed to embed the particular $\mathrm{U}(1)$ in a larger group. Such that focusing on the $\mathrm{U}(1)$ of the SSM one finds

$$
b_{1}=\frac{3}{5}\left(\frac{2}{3} \sum_{f} Y_{f}^{2}+\frac{1}{3} \sum_{s} Y_{s}^{2}\right) \quad \text { or } \quad b_{1}=\frac{3}{5}\left(\sum_{\Phi} Y_{\Phi}^{2}\right),
$$




\begin{tabular}{|c|c|c|c|}
\hline Superfields & Brane & Bulk & $\mathrm{U}(1)_{Y} \times \mathrm{SU}(2)_{L} \times \mathrm{SU}(3)_{c}$ \\
\hline$\hat{q}^{1,2}$ & $\checkmark$ & - & $\left(\frac{1}{6}, \mathbf{2}, \mathbf{3}\right)$ \\
$\hat{d}^{1,2}$ & $\checkmark$ & - & $\left(\frac{1}{3}, \mathbf{1}, \overline{\mathbf{3}}\right)$ \\
$\hat{u}^{1,2}$ & $\checkmark$ & - & $\left(-\frac{2}{3}, \mathbf{1}, \overline{\mathbf{3}}\right)$ \\
$\hat{l}^{1,2}$ & $\checkmark$ & - & $\left(-\frac{1}{2}, \mathbf{2}, \mathbf{1}\right)$ \\
$\hat{e}^{1,2}$ & $\checkmark$ & - & $(1, \mathbf{1}, \mathbf{1})$ \\
\hline$\hat{q}^{3}$ & - & $\checkmark$ & $\left(\frac{1}{6}, \mathbf{2}, \mathbf{3}\right)$ \\
$\hat{d}^{3}$ & - & $\checkmark$ & $\left(\frac{1}{3}, \mathbf{1}, \overline{\mathbf{3}}\right)$ \\
$\hat{u}^{3}$ & - & $\checkmark$ & $\left(-\frac{2}{3}, \mathbf{1}, \overline{\mathbf{3}}\right)$ \\
$\hat{l}^{3}$ & - & $\checkmark$ & $\left(-\frac{1}{2}, \mathbf{2}, \mathbf{1}\right)$ \\
$\hat{e}^{3}$ & - & $\checkmark$ & $(1, \mathbf{1}, \mathbf{1})$ \\
\hline$\hat{H}_{d}$ & - & $\checkmark$ & $\left(-\frac{1}{2}, \mathbf{2}, \mathbf{1}\right)$ \\
$\hat{H}_{u}$ & - & $\checkmark$ & $\left(\frac{1}{2}, \mathbf{2}, \mathbf{1}\right)$ \\
\hline$\hat{F}_{-}$ & - & $\checkmark$ & $(-1, \mathbf{1}, \mathbf{1})$ \\
$\hat{F}_{+}$ & - & $\checkmark$ & $(1, \mathbf{1}, \mathbf{1})$ \\
\hline$\hat{B}_{V}$ & - & $\checkmark$ & $(0, \mathbf{1}, \mathbf{1})$ \\
$\hat{W}_{V}$ & - & $\checkmark$ & $(0, \mathbf{3}, \mathbf{1})$ \\
$\hat{G}_{V}$ & - & $\checkmark$ & $(0, \mathbf{1}, \mathbf{8})$ \\
\hline
\end{tabular}

Table 2. The matter content of model 2. In this case the third generation also lives in the bulk. The gauge couplings of this model unify as in figure 1 (top right).

the latter is for chiral superfields, and the $Y$ 's are hypercharges, where the hypercharge is rescaled by $g_{1} \equiv \sqrt{5 / 3} g^{\prime}$ as usual in unified models [31]. The results for various models may be found in table 4 , where we note that unification scales of the order of $10 \mathrm{TeV}$ can still satisfy proton decay constraints, this conclusion being applicable to a wide range of gauge groups or extra-dimensional models [32]. In a number of these scenarios additional matter is required to obtain unification, or indeed the extra dimensional scale $1 / R>10^{10} \mathrm{GeV}$, which for phenomenological purposes is essentially four dimensional and so not of interest.

A useful comment is appropriate here that the additional matter of the $5 D$ MSSM-UED scenario means that all beta function coefficients are positive. This forces $1 / R \gtrsim 10^{10} \mathrm{GeV}$ for unification to still be possible [35]. Low scale (supersymmetric) extra dimensions therefore require that most of the MSSM matter does not live in the bulk. Our preferred scenarios are therefore ones in which the matter multiplets all live on a brane (model 1 ) or where the 1st and 2nd generation live on an opposite brane to the 3rd generation, or where only the third generation lives in the bulk (model 2), or where only the third generation lives in the brane (model 3, see table 3 for the matter content in this case). In either case the Higgses can live in the bulk or on a brane. Additional fields may be added to accomplish precision unification at low scales [39]. This leads to three options: the models 1, 2 and 3 that we consider in this paper (plotted in figure 1) and one might also be able to combine a 


\begin{tabular}{|c|c|c|c|}
\hline Superfields & Brane & Bulk & $\mathrm{U}(1)_{Y} \times \mathrm{SU}(2)_{L} \times \mathrm{SU}(3)_{c}$ \\
\hline$\hat{q}^{1,2}$ & - & $\checkmark$ & $\left(\frac{1}{6}, \mathbf{2}, \mathbf{3}\right)$ \\
$\hat{d}^{1,2}$ & - & $\checkmark$ & $\left(\frac{1}{3}, \mathbf{1}, \overline{\mathbf{3}}\right)$ \\
$\hat{u}^{1,2}$ & - & $\checkmark$ & $\left(-\frac{2}{3}, \mathbf{1}, \overline{\mathbf{3}}\right)$ \\
$\hat{l}^{1,2}$ & - & $\checkmark$ & $\left(-\frac{1}{2}, \mathbf{2}, \mathbf{1}\right)$ \\
$\hat{e}^{1,2}$ & - & $\checkmark$ & $(1, \mathbf{1}, \mathbf{1})$ \\
\hline$\hat{q}^{3}$ & $\checkmark$ & - & $\left(\frac{1}{6}, \mathbf{2}, \mathbf{3}\right)$ \\
$\hat{d}^{3}$ & $\checkmark$ & - & $\left(\frac{1}{3}, \mathbf{1}, \overline{\mathbf{3}}\right)$ \\
$\hat{u}^{3}$ & $\checkmark$ & - & $\left(-\frac{2}{3}, \mathbf{1}, \overline{\mathbf{3}}\right)$ \\
$\hat{l}^{3}$ & $\checkmark$ & - & $\left(-\frac{1}{2}, \mathbf{2}, \mathbf{1}\right)$ \\
$\hat{e}^{3}$ & $\checkmark$ & - & $(1, \mathbf{1}, \mathbf{1})$ \\
\hline$\hat{H}_{d}$ & - & $\checkmark$ & $\left(-\frac{1}{2}, \mathbf{2}, \mathbf{1}\right)$ \\
$\hat{H}_{u}$ & - & $\checkmark$ & $\left(\frac{1}{2}, \mathbf{2}, \mathbf{1}\right)$ \\
\hline$\hat{F}_{-}$ & - & $\checkmark$ & $(-1, \mathbf{1}, \mathbf{1})$ \\
$\hat{F}_{+}$ & - & $\checkmark$ & $(1, \mathbf{1}, \mathbf{1})$ \\
\hline$\hat{B}_{V}$ & - & $\checkmark$ & $(0, \mathbf{1}, \mathbf{1})$ \\
$\hat{W}_{V}$ & - & $\checkmark$ & $(0, \mathbf{3}, \mathbf{1})$ \\
$\hat{G}_{V}$ & - & $\checkmark$ & $(0, \mathbf{1}, \mathbf{8})$ \\
\hline
\end{tabular}

Table 3. The matter content of model 3. In this case the 1st and 2 nd generation live in the bulk. The gauge couplings of this scenario do not unify, as in figure 1 (bottom).

4D M-Dirac-SSM [38] with a maximal super Yang-Mills theory only in the bulk [26], rather remarkably, to achieve unification for any and all sizes of inverse radius. In this theory the gauge couplings only run in the four dimensional theory as the beta functions for the gauge couplings vanish exactly to all orders in perturbation theory in the maximal super Yang-Mills theory. As a result there are no power law contributions for gauge couplings (but there may be for the Yukawas and soft terms) and an inverse radius of a few $\mathrm{TeV}$ is possible with gauge coupling unification at $10^{17} \mathrm{GeV}$, which is very counter-intuitive. The effective cutoff of a five dimensional theory is essentially defined as the scale at which some parameter, such as the gauge couplings, hit a Landau pole: as no Landau pole arises this allows for the range of validity of this theory to extend further.

\section{Exploring the models}

In this section we explore the typical scales of the models, we describe how we solve the various RGEs and the boundary conditions that we use and then look at many of the running parameters of the model, such as trilinear soft breaking parameters and the gaugino mass spectrum. 


\begin{tabular}{|c|c|c|c|}
\hline Scenario & $\left(b_{1}, b_{2}, b_{3}\right)$ & Refs: & $1 / R$-GUT \\
\hline $4 D$ SM & $\left(\frac{41}{10},-19 / 6,-7\right)$ & & - \\
$4 D$ MSSM & $\left(\frac{33}{5}, 1,-3\right)$ & {$[33,34]$} & - \\
$5 D$ MSSM: Chiral Higgses in the bulk & $\left(\frac{3}{5},-3,-6\right)$ & {$[20,29]$} & $\star$ (does not exist) \\
$5 D$ MSSM: Hyper Higgses in the bulk & $\left(\frac{6}{5},-2,-6\right)$ & {$[18,27]$} & $\sim 10^{10} \mathrm{GeV}$ \\
$5 D$ MSSM-UED & $\left(\frac{66}{5}, 10,6\right)$ & {$[35,36]$} & $\geq 5 \times 10^{10} \mathrm{GeV}$ \\
$5 D$ 3rd Gen \& Hyper Higgses in the bulk & $\left(\frac{26}{5}, 2,-2\right)$ & {$[37]$} & $\sim 10^{10} \mathrm{GeV}$ \\
$5 D$ 1st,2nd Gen \& Hyper Higgses in the bulk & $\left(\frac{40}{5}, 4,2\right)$ & & $\boldsymbol{X}$ \\
$5 D$ Gauge only in the bulk & $(0,-4,-6)$ & & $\sim 10^{10} \mathrm{GeV}$ \\
\hline $4 D S S M F^{ \pm}$ & $\left(\frac{39}{5}, 1,-3\right)$ & & - \\
$5 D S S M F^{ \pm}:$Hyper Higgses in bulk & $\left(\frac{18}{5},-2,-6\right)$ & model 1 & $\geq 1 \mathrm{TeV}$ \\
$5 D S S M F^{ \pm}: 3$ rd Gen \& Hyper Higgses in bulk & $\left(\frac{38}{5}, 2,-2\right)$ & model 2 & $\geq 1 \mathrm{TeV}$ \\
$5 D S S M F^{ \pm}: 1$ st,2nd Gen \& Hyper Higgses in bulk & $\left(\frac{58}{5}, 6,2\right)$ & model 3 & $\geq 1 \mathrm{TeV}$ \\
\hline $4 D$ MSSM+Dirac & $\left(\frac{33}{5},-1,0\right)$ & & - \\
$4 D$ M-Dirac-SSM & $\left(\frac{48}{5}, 4,0\right)$ & {$[38]$} & - \\
$5 D$ MSYM only in the bulk & $(0,0,0)$ & {$[26]$} & any \\
$5 D$ MSYM Hyper-Higgs in the bulk & $\left(\frac{6}{5}, 2,0\right)$ & & $\boldsymbol{X}$ \\
\hline
\end{tabular}

Table 4. The one-loop beta function coefficients of the gauge couplings for various scenarios. Requiring gauge coupling unification puts a bound on the inverse radius of the extra dimension in five dimensional models, which is estimated in the right-most column.

\subsection{Typical scales of the models}

It is useful to set the mass and energy scales in which we wish to consider these models. We wish for a large extra dimension, which then leads us to fix the gauge coupling unification scale and the scale of the cut off, where the gauge couplings hit a Landau pole (see figure 1):

$$
\frac{1}{R} \sim 10 \mathrm{TeV}, \quad M_{\mathrm{GUT}} \sim 300 \mathrm{TeV}, \quad \Lambda \sim 1,000 \mathrm{TeV} .
$$

Although they differ in magnitude, this is natural in that fixing any one of these determines the other two. Next we wish for a gluino mass above collider exclusions and to determine the Higgs mass correctly to be $m_{h}=125 \mathrm{GeV}$ from a sizeable $A_{t}$. We find (see for instance figure 3)

$$
M_{3}=900 \mathrm{GeV} \text { leads to } A_{t} \sim-700 \mathrm{GeV}, \quad M_{3}=1700 \mathrm{GeV} \text { leads to } A_{t} \sim-1250 \mathrm{GeV} \text {. }
$$

Strong exclusion limits on the gluino arise from ATLAS and CMS null searches for jets plus missing energy, for example $m_{\tilde{g}}>1600 \mathrm{GeV}$ for $m_{\tilde{q}_{1,2}}>2000 \mathrm{GeV}$ [40, 41], although this can be lowered if one wishes to also include R-parity violation with our models, hence the $M_{3}=900 \mathrm{GeV}$ case. Conversely, allowing for an upper bound on the top trilinear coupling, from considering metastability of the electroweak vacuum,

$$
A_{t}=-2 \mathrm{TeV} \text { leads to } M_{3} \sim 2.77 \mathrm{TeV} \quad \text { and } \quad A_{t}=-2.5 \mathrm{TeV} \text { leads to } M_{3} \sim 3.5 \mathrm{TeV} \text {. }
$$




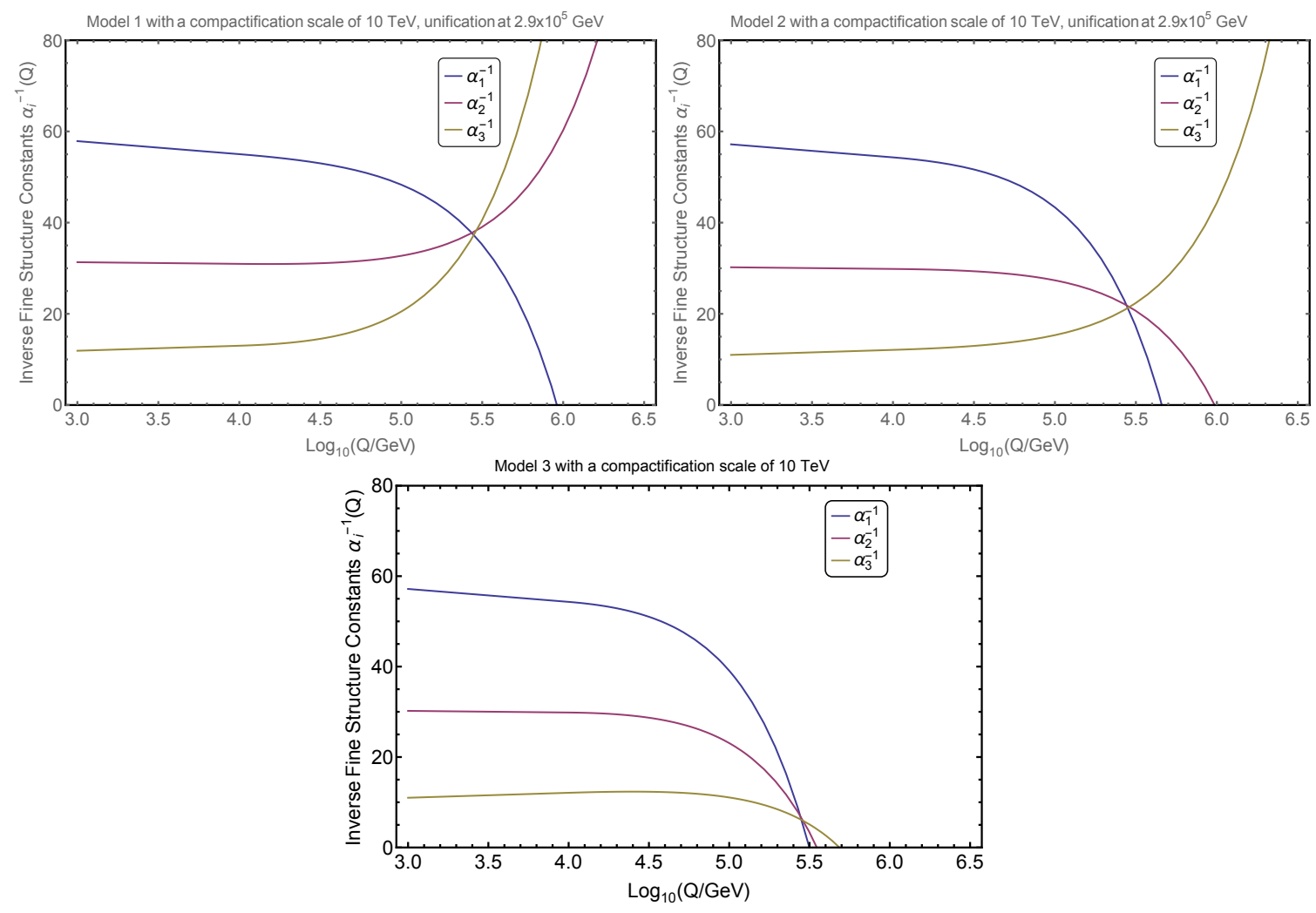

Figure 1. Running of the inverse fine structure constants $\alpha_{i}^{-1}(Q)$, for three different models with compactification scales $10 \mathrm{TeV}$ as a function of $\log _{10}(\mathrm{Q} / \mathrm{GeV})$.

To allow for the correct Higgs mass $m_{h}=125 \mathrm{GeV}$, the electroweak parameters should be in the range

$$
\tan \beta \subset(5,30), \quad \mu \leq 1 \mathrm{TeV},
$$

represented in figure 6 . We do not expect $\tan \beta$ to be much larger, due to $B_{s} \rightarrow X_{s} \gamma$ flavour constraints and $\mu$ is bounded by naturalness considerations of the renormalisation group effects on the Higgs tadpole equations (minimisation of the scalar potential).

\subsection{Implementation and results}

To obtain our results we computed by hand the various RGEs of the four dimensional (zero mode) description that both model 1 and 2 (tables 1 and 2) reduce to at low energies. We then confirmed these with the output of an implementation of the four dimensional regime in SARAH [42-45]. We then computed, by hand only, the one-loop RGEs for each of model 1, 2 and 3, including all the additional fields of the KK sector. Using MATHEMATICA we solve the combined set of RGEs and match the four and five dimensional RGEs at the matching compactification scale such that at low energies the theory is described by the four dimensional RGEs only.

Once we have a combined set of RGEs, we must specify a set of boundary conditions. In this case we must simply specify all boundary conditions at the same scale (rather than, for example, having a set of boundary conditions at both the GUT/SUSY-breaking scale and at the electroweak scale), which we took to be $t=3$, or $Q=10^{3} \mathrm{GeV}$ (where we define 

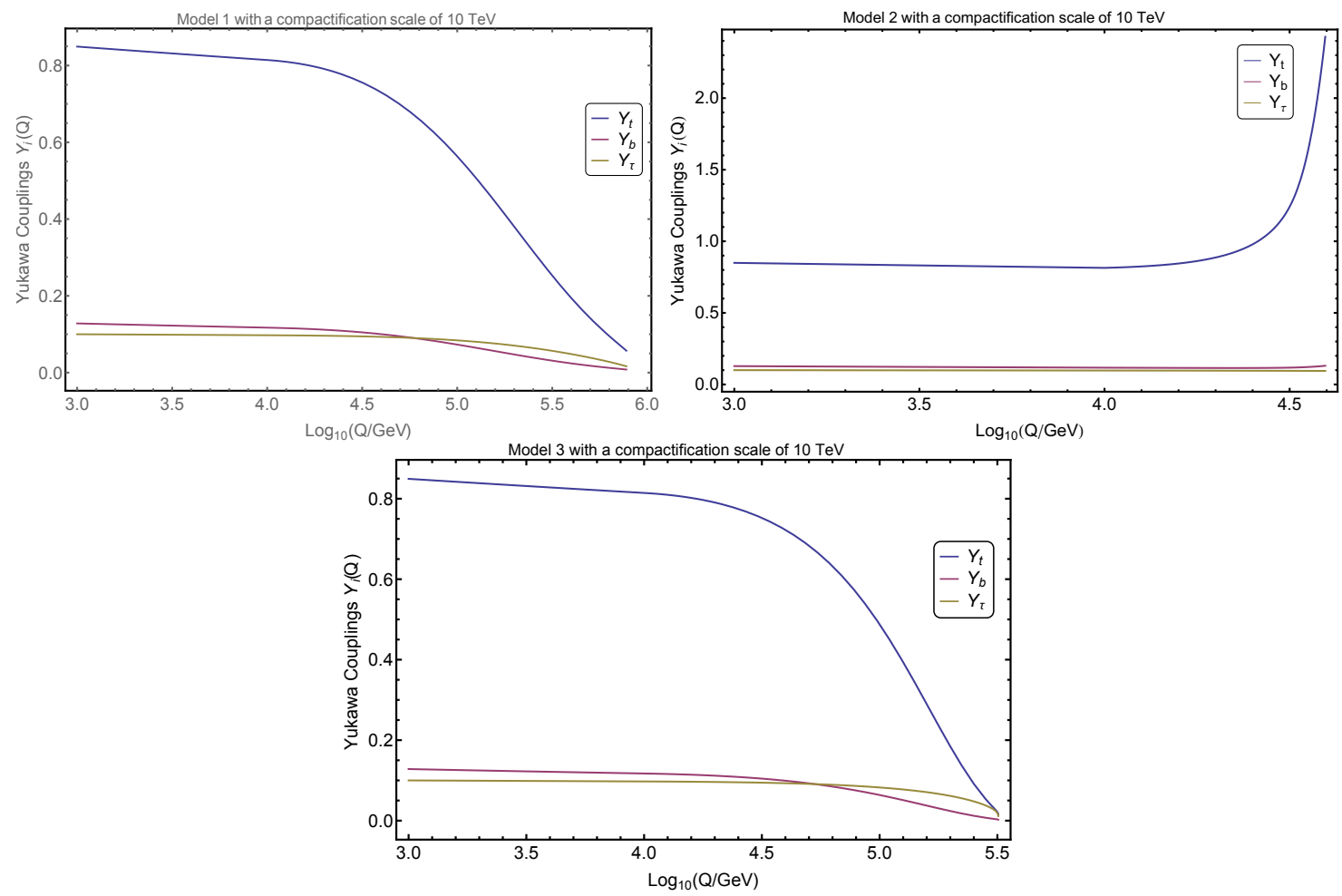

Figure 2. Running of the Yukawa couplings $Y_{i}(Q)$, for thee different models with compactification scales $10 \mathrm{TeV}$ as a function of $\log _{10}(\mathrm{Q} / \mathrm{GeV})$. The top Yukawa coupling typically hits a Landau pole before the GUT scale when the 3rd generation matter is located in the bulk (right).

\begin{tabular}{|c|c|c|}
\hline Parameter & Value & Name \\
\hline$Q_{0}$ & 1000 & (SUSY Scale) \\
$g_{1}\left(Q_{0}\right)$ & 0.360945804 & $\mathrm{~g} 1$ \\
$g_{2}\left(Q_{0}\right)$ & 0.633371083 & $\mathrm{~g} 2$ \\
$g_{3}\left(Q_{0}\right)$ & 1.02739852 & $\mathrm{~g} 3$ \\
\hline $\tan \beta$ & 10 & (Tan beta) \\
$Y_{t}\left(Q_{0}\right)$ & 0.849348847 & (Top Yukawa) \\
$Y_{b}\left(Q_{0}\right)$ & 0.128188819 & (Bottom Yukawa) \\
$Y_{\tau}\left(Q_{0}\right)$ & 0.0999653768 & (Tau Yukawa) \\
\hline
\end{tabular}

Table 5. A table of the boundary conditions used in our study.

$\left.t=\log _{10} Q\right)$. The gauge couplings and Yukawa couplings are easily obtained by running up from $m_{Z}$ and are listed in table 5 , for example in figure 2 for $\tan \beta \sim 10$. Regarding the soft breaking terms we made some specific choices which we enforce by choosing a low-scale boundary value such that it holds true once we run up to the high scale. We also make the assumption that the SUSY breaking scale is equal to the GUT scale, but of course other scenarios should be considered. For model 1 and 3: 
- We assume supersymmetry breaking occurs at the unification scale, which is found by finding the scale at which $g_{1}=g_{2}=g_{3}$, which is lowered compared to the $4 \mathrm{D}$ MSSM, by the effects of the compactification. This is pictured in figure 1 (top left).

- We specify the value of the gluino mass, $M_{3}(Q)$, at $Q=10^{3} \mathrm{GeV}$. We then find the bino and wino soft masses $M_{1}$ and $M_{2}$ such that all gaugino masses $M_{1}=M_{2}=M_{3}$ at the GUT scale. This is pictured in figure 3.

- We take the trilinear soft breaking terms, $A_{u / d / e}$, to vanish at the unification scale, also pictured in figure 3 .

- We take $\mu(t=3) \sim 500 \mathrm{GeV}$ and $B_{\mu}\left(M_{\mathrm{GUT}}\right)=0$, as pictured in figure 5 (left).

The results are rather different for model 2:

- We found the scale at which $g_{1}=g_{2}=g_{3}$, which is lowered compared to the $4 \mathrm{D}$ MSSM, pictured in figure 1 (top right).

- The top Yukawa coupling hits a Landau pole just after $t=4.595$, as pictured in figure 2 (right).

The result was that we could not set the supersymmetry breaking scale at $M_{\mathrm{GUT}}$ and instead chose the supersymmetry breaking scale to occur below the top Yukawa Landau pole, at $t=4.4$. We then chose for the plots in model 2:

- We choose the gaugino masses to unify $M_{3}(t=4.4)=M_{2}(t=4.4)=M_{1}(t=4.4)$ and let $M_{3}(t=3)=1700 \mathrm{GeV}$.

- $A_{u / d / e}(t=4.4)$ are set to vanish and this model does not develop a TeV scale $A_{t}(t=$ 3 ), as pictured in figure 3 (right).

- Whilst electroweak symmetry breaking is possible starting from the condition $m_{H_{d}}^{2}=$ $m_{H_{u}}^{2}$, it does not automatically arise from using $\left(m_{0}^{2}+\mu^{2}\right)^{1 / 2}$, where $m_{0}^{2}$ would set the scalar soft mass boundary condition. This is pictured in figure 4 (right), where a representative case is given that achieves the correct Higgs mass.

- We take $\mu(t=3) \sim 500 \mathrm{GeV}$ and $B_{\mu}\left(M_{\mathrm{GUT}}\right)=0$, as pictured in figure 5 (left).

\subsection{Two ways to accommodate natural supersymmetry}

The two models we explore in this paper can accommodate a natural spectrum of sparticles in two very different ways, whilst still obtaining the correctly observed Higgs mass:

In model 2 the third generation are located in the bulk and feel the effects of supersymmetry more indirectly than the first and second generation. This will allow for a spectrum of light stops with a heavier first and second generation, above present collider exclusions. One may use the NMSSM or D-terms to lift the Higgs mass to its correct value.

In model 1 the Higgs mass is obtained through a TeV scale $A_{t}$ term that is generated entirely through RGE evolution, allowing for the correct Higgs mass with stops much below 

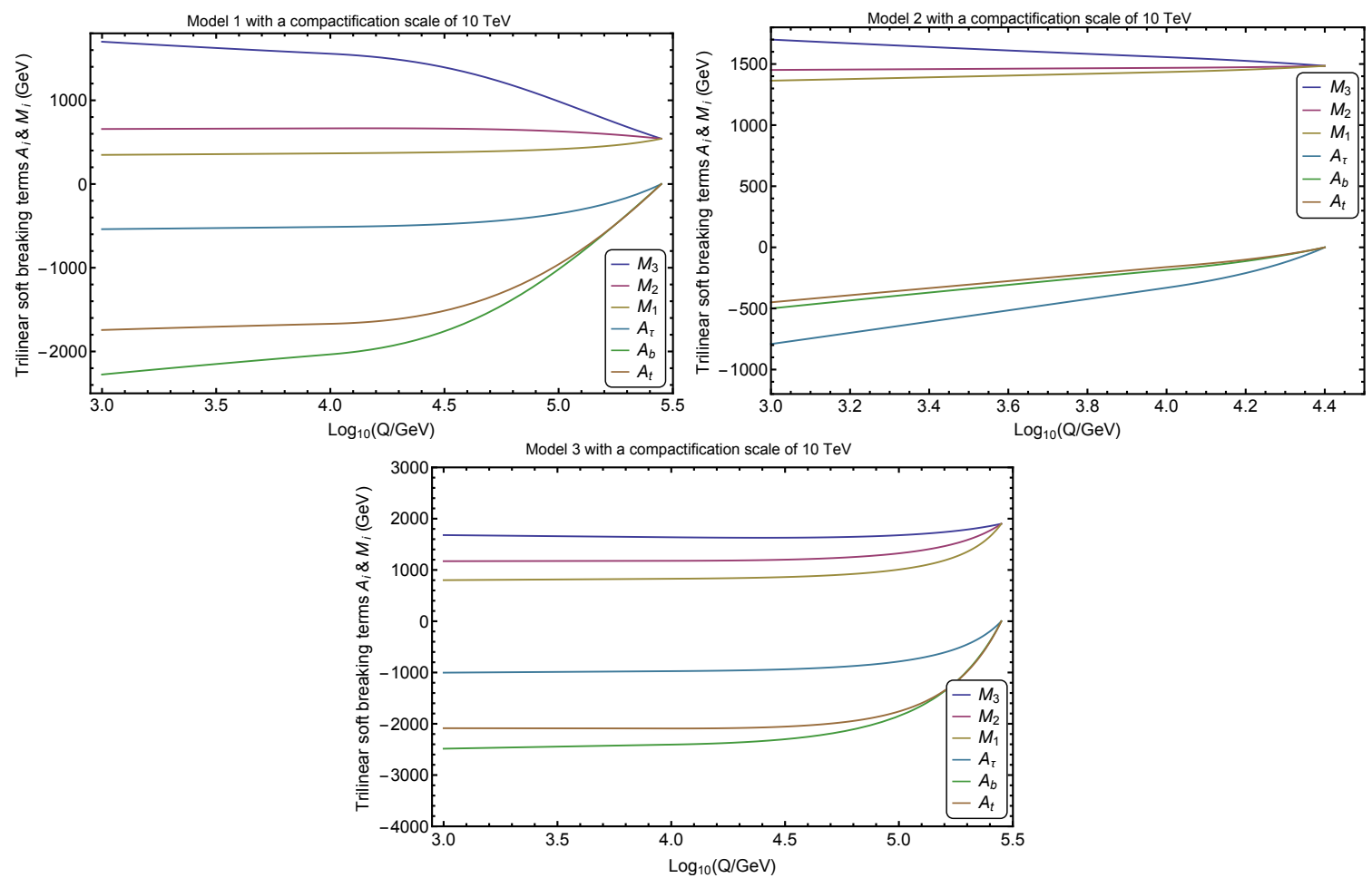

Figure 3. Running of the gaugino masses and trilinear couplings $M_{i}(Q)$ and $A_{i}(Q)$, for the two different models with compactification scales $10 \mathrm{TeV}$, as a function of $\log _{10}(\mathrm{Q} / \mathrm{GeV})$.

$2 \mathrm{TeV}$ even within an MSSM-like Higgs sector, but does not yet explain any hierarchy between the generation of squarks. In this subsection we explain these details of each model further.

\subsubsection{The third generation in the bulk}

Exclusions on first and second generation squarks are presently nearing $2 \mathrm{TeV}$ [46-53], while the aesthetic of naturalness for the Higgs sector (and much weaker bounds on 3rd generation squarks of around 300-400 GeV [7-17] from direct searches) favour a 3rd generation below a TeV. In order for this hierarchy to emerge at low scales it is likely to be imprinted in the soft SUSY breaking terms and not simply a renormalisation group effect. At the supersymmetry breaking scale this might imply that the soft terms, in the flavour basis, take the form,

$$
m_{\tilde{f}}^{2} \sim \Lambda^{2}\left(\begin{array}{lll}
1 & 0 & 0 \\
0 & 1 & 0 \\
0 & 0 & 0
\end{array}\right)+\ldots
$$

or indeed

$$
m_{\tilde{f}}^{2} \sim \Lambda^{2}\left(\begin{array}{ccc}
1 & 0 & 0 \\
0 & 1 & 0 \\
0 & 0 & -\varepsilon
\end{array}\right)+\ldots
$$



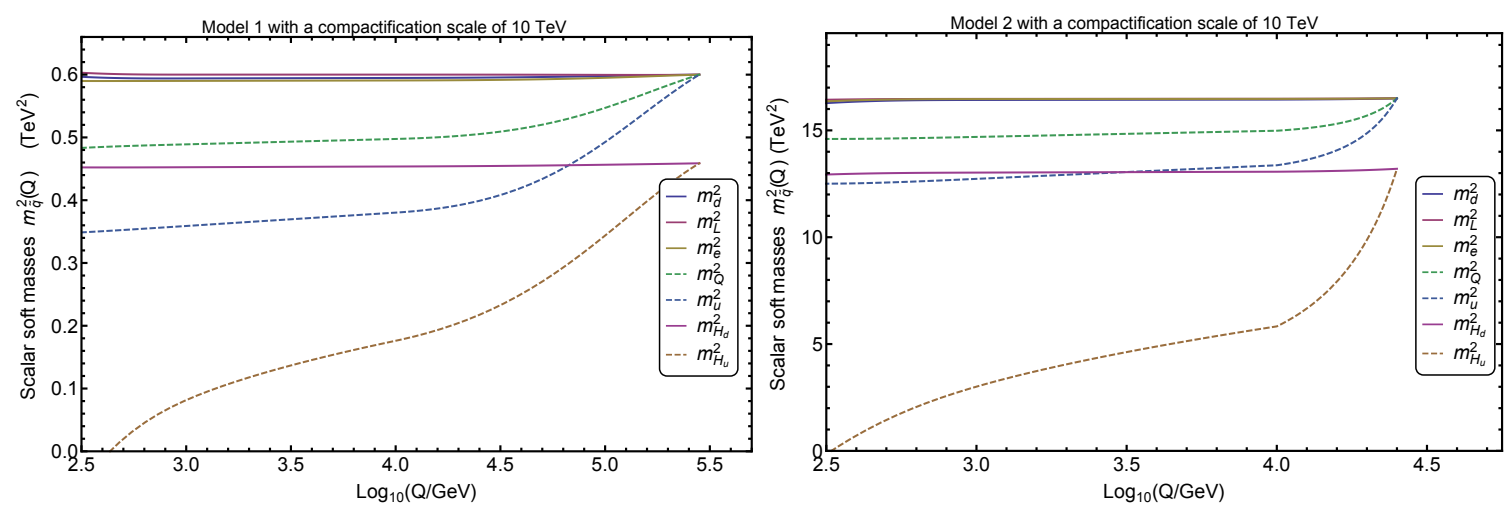

Figure 4. Running of the various soft masses for the two models.

the $\varepsilon$ denoting any subleading effects, as one does not require exactly zero entries. Some ideas have been put forward to explain such a heirarchy, see for instance $[5,18,54,55]$, and we wish to advance the argument that a five dimensional model with the 3rd generation in the bulk, i.e our model 2, explains such a hierarchy.

We put forward the idea that the first and second generation of squarks live on the same brane as the source of supersymmetry breaking. They will feel directly the effect of supersymmetry breaking and generate large soft breaking terms. The 3rd generation is, however, located in the bulk and will feel the supersymmetry breaking indirectly through either gravity or gauge mediation. This will lead to the boundary conditions in eq. (3.5). For a calculation of gauge mediated soft terms from a brane to a bulk field see [24,56], for brane to other brane see [23, 24, 56]. Such an effect is still felt directly by the gauginos (and the gravitino) and they will also have a large SUSY breaking soft mass, which have important RGE effects as discussed in this paper.

\subsubsection{A large $A_{t}$ term}

Our model 1 does not geometrically explain why the first and second generation might be much heavier than the $3 \mathrm{rd}$, but it does allow for a large $A_{t}$ term generated entirely through RGE evolution, and this can still allow for stops much below $2 \mathrm{TeV}$ and still obtain the correct Higgs mass from the usual MSSM Higgs sector. Therefore for model 1, we do not yet offer an explanation of the source of supersymmetry breaking. We discuss obtaining the correct Higgs mass in model 1 in the next section.

\section{The Higgs mass}

The Higgs mass is a sensitive parameter in supersymmetric theories and its experimental value at $125 \mathrm{GeV}$ restricts the available parameter space for these models. A realistic and precise calculation of the Higgs mass in supersymmetric models requires the inclusion of two-loop contributions. In the following we shall use the numerical values of the program FeynHiggs 2.11.3 $[57,58]$ for the Higgs mass at two-loops and interface it with our numerical code for the models 1 and 2 we discussed above. As the Higgs mass is a low energy 

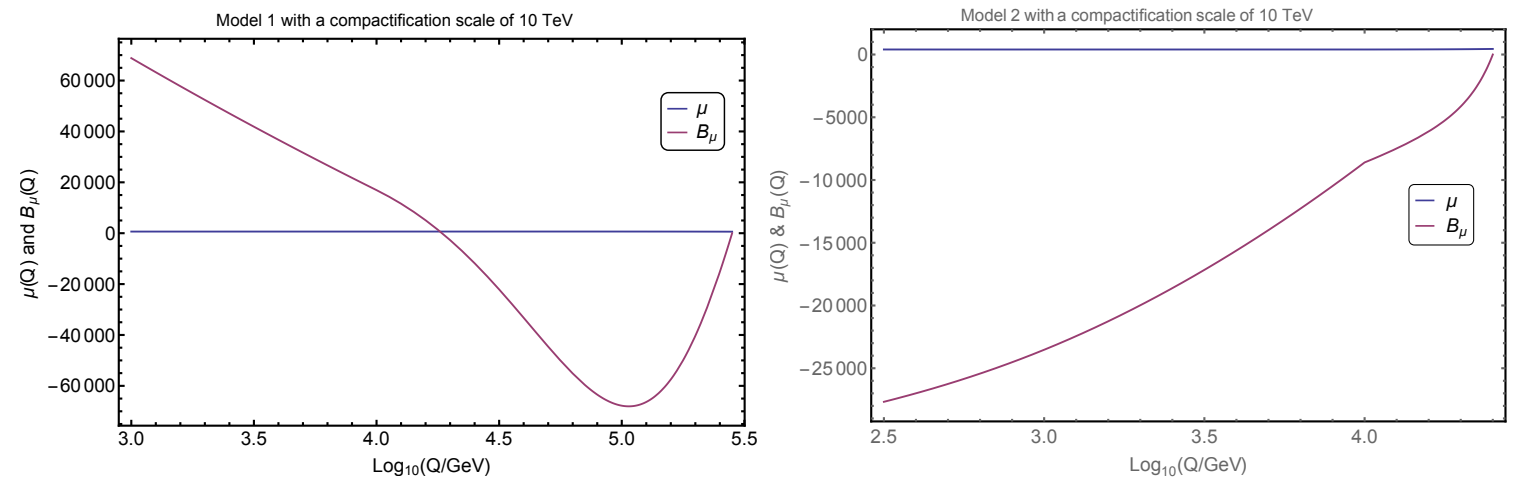

Figure 5. The running of $\mu$ and $B_{\mu}$ for the two models.

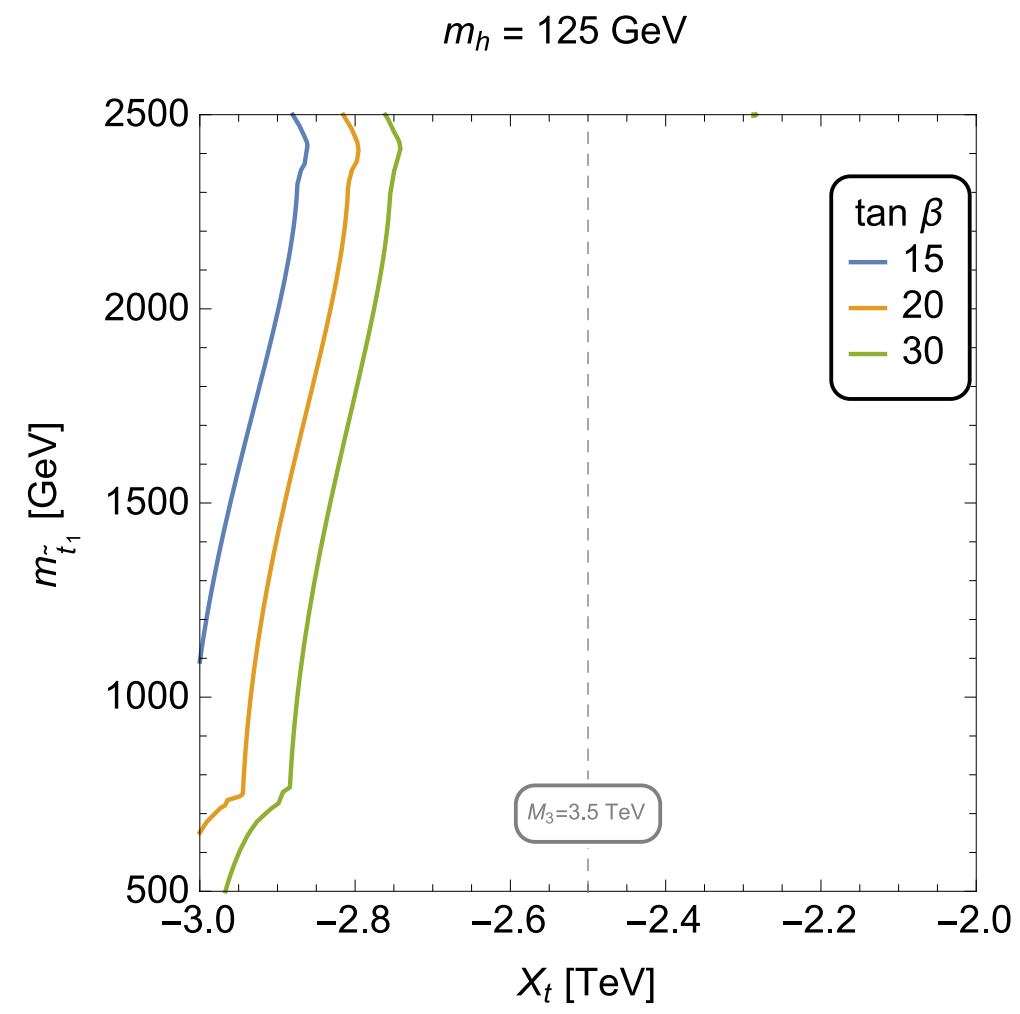

Figure 6. Contours of the lightest Higgs mass $m_{h}=125 \mathrm{GeV}$ in the plane $\left(m_{\tilde{t}_{1}}, X_{t}\right)$ for various values of $\tan \beta$. The dashed gray line represents a sample gluino mass for the corresponding value of $X_{t}$. Stop masses below $2 \mathrm{TeV}$ can be obtained in our model due to the TeV-scale $A_{t}$ term.

parameter with respect to the extra-dimensional contributions, the corrections due to the extra-dimensional structure are very small in the plot in figure 6 . In order to have a rough physical intuition concerning the effect of the large $A_{t}$ value from our setup, we provide here the leading one-loop self-energy contributions to the lightest CP even Higgs mass [59-63]

$$
m_{h, 1}^{2} \simeq m_{z}^{2} \cos ^{2} 2 \beta+\frac{3}{4 \pi^{2}} \frac{m_{t}^{4}}{v_{\mathrm{ew}}^{2}}\left[\ln \frac{M_{S}^{2}}{m_{t}^{2}}+\frac{X_{t}^{2}}{M_{S}^{2}}\left(1-\frac{X_{t}^{2}}{12 M_{S}^{2}}\right)\right]
$$


where $v_{\text {ew }}$ is the electroweak Higgs vev, $X_{t}=A_{t}-\mu \cot \beta$ and $M_{S}^{2}=m_{\tilde{t}_{1}} m_{\tilde{t}_{2}}$. If $\mu$ is a few $100 \mathrm{GeV}$ and $A_{t} \gg \mu$ then $X_{t} \sim A_{t}$. The result of the one-loop formula allows to understand why a model with large $A_{t}$ values can help increasing the value of the Higgs mass. Note however that the one-loop formula is not precise enough to for a realistic comparison with data and typically allows a larger parameter space that what really available including the two-loop formulas. We plot, in figure 6, the Higgs mass for representative values of $A_{t}$ in our setup using the FeynHiggs program at two-loops. This allows for a prediction of $\tan \beta$ and the stop squark masses which can be below $2 \mathrm{TeV}$. One could also lift the tree-level Higgs mass with the NMSSM $+F^{ \pm}$or else through non decoupling D-terms (see for example $[5,6]$ ) which would require introducing an additional $\mathrm{U}(1)$ or $\mathrm{SU}(2)$ or both. Such an additional feature would be necessary for model 2 as a large $A_{t}$ does not arise in this case.

\section{Conclusions}

In this paper we explored various five dimensional extensions of the SSM that unify, with an inverse radius of the extra dimension of roughly a $10 \mathrm{TeV}$ scale. Such models are compelling extensions of the MSSM in that they may achieve interesting phenomenological features such as additional $Z^{\prime}, W^{\prime}$ and $G^{\prime}$ bosons in the $1-10 \mathrm{TeV}$ range and achieve the correct $125 \mathrm{GeV}$ Higgs mass and a relatively natural sparticle spectrum for model 1, while for model 2 this spectrum is heavier, without sacrificing unification of gauge couplings. Such models achieve a natural spectrum by generating a TeV scale $A_{t}$ term from "power-law" running and unification of gauge couplings through the addition of two charged superfields $F^{ \pm}$in the bulk.

In particular we look at two models that can achieve unification, either all chiral matter superfields on the boundaries, or just the third generation in the bulk and the first two on a boundary. In either case the Higgs doublet superfields $H_{u}, H_{d}$ and $F^{ \pm}$are located in the bulk along with all three gauge groups $\mathrm{SU}(3)_{c} \times \mathrm{SU}(2)_{L} \times \mathrm{U}(1)_{Y}$. We also point out that five dimensional models in which the 1 st and 2 nd generation are located in the bulk cannot possibly achieve unification unless the inverse radius of the extra dimension is essentially at the GUT scale (and in any case not with this matter content), and so are entirely four dimensional from a phenomenological perspective.

This paper can be extended in a number of ways and we discuss just a few. In many models of supersymmetry breaking, electroweak symmetry breaking is not optimal both in terms of fine tuning and in obtaining electroweak breaking from a given parameterisation of soft breaking terms at the high scale. These remain an interesting open question, and may benefit from further discoveries or exclusions in the Higgs sector, at the LHC13/14. Our results are representative only, and clearly a more dedicated spectrum generator built using the RGEs and including threshold corrections will give more precise results, and we provide in this paper a concrete set of RGEs from which this spectrum generator can be constructed. Different supersymmetry breaking parameterisations and how flavour arises is also an interesting further direction to consider. 


\section{Acknowledgments}

This work was partially supported by the Foundation for Polish Science International PhD Projects Programme co-financed by the EU European Regional Development Fund. This work has been partially supported by National Science Centre under research grant DEC2012/04/A/ST2/00099. AD is partially supported by Institut Universitaire de France. We also acknowledge partial support from the Labex-LIO (Lyon Institute of Origins) under grant ANR-10-LABX-66 and FRAMA (FR3127, Fédération de Recherche "André Marie Ampère"). This work is supported by the National Research Foundation (South Africa).

\section{A Renormalisation group equations for $4 \mathrm{D}-\mathrm{SSM}+\boldsymbol{F}^{ \pm}$}

In this appendix we document the one- and two-loop RGEs for the four dimensional low energy model for which the 5D models 1, 2 and 3 are completions. Recall that the output of our implementation in the four dimensional regime was done using SARAH [42-45], as such we have used the same conventions and notations, where $T_{i}=A_{i} y_{i}(i=t, b, \tau$ etc. $)$ in these appendices.

\section{A.1 Anomalous dimensions}

$$
\begin{aligned}
\gamma_{\hat{q}}^{(1)}= & -\frac{1}{30}\left(45 g_{2}^{2}+80 g_{3}^{2}+g_{1}^{2}\right) \mathbf{1}+Y_{d}^{\dagger} Y_{d}+Y_{u}^{\dagger} Y_{u} \\
\gamma_{\hat{q}}^{(2)}= & +\frac{1}{180}\left(2 g_{1}^{2}\left(16 g_{3}^{2}+9 g_{2}^{2}\right)+47 g_{1}^{4}+5\left(135 g_{2}^{4}+288 g_{2}^{2} g_{3}^{2}-32 g_{3}^{4}\right)\right) \mathbf{1} \\
& +\frac{4}{5} g_{1}^{2} Y_{u}^{\dagger} Y_{u}-2 Y_{d}^{\dagger} Y_{d} Y_{d}^{\dagger} Y_{d}-2 Y_{u}^{\dagger} Y_{u} Y_{u}^{\dagger} Y_{u} \\
& +Y_{d}^{\dagger} Y_{d}\left(-3 \operatorname{Tr}\left(Y_{d} Y_{d}^{\dagger}\right)+\frac{2}{5} g_{1}^{2}-\operatorname{Tr}\left(Y_{e} Y_{e}^{\dagger}\right)\right)-3 Y_{u}^{\dagger} Y_{u} \operatorname{Tr}\left(Y_{u} Y_{u}^{\dagger}\right) \\
\gamma_{\hat{l}}^{(1)}= & -\frac{3}{10}\left(5 g_{2}^{2}+g_{1}^{2}\right) \mathbf{1}+Y_{e}^{\dagger} Y_{e} \\
\gamma_{\hat{l}}^{(2)}= & -2 Y_{e}^{\dagger} Y_{e} Y_{e}^{\dagger} Y_{e}+\frac{3}{100}\left(125 g_{2}^{4}+30 g_{1}^{2} g_{2}^{2}+81 g_{1}^{4}\right) \mathbf{1} \\
& +Y_{e}^{\dagger} Y_{e}\left(-3 \operatorname{Tr}\left(Y_{d} Y_{d}^{\dagger}\right)+\frac{6}{5} g_{1}^{2}-\operatorname{Tr}\left(Y_{e} Y_{e}^{\dagger}\right)\right) \\
\gamma_{\hat{H}_{d}}^{(1)}= & 3 \operatorname{Tr}\left(Y_{d} Y_{d}^{\dagger}\right)-\frac{3}{10}\left(5 g_{2}^{2}+g_{1}^{2}\right)+\operatorname{Tr}\left(Y_{e} Y_{e}^{\dagger}\right) \\
\gamma_{\hat{H}_{d}}^{(2)}= & +\frac{243}{100} g_{1}^{4}+\frac{9}{10} g_{1}^{2} g_{2}^{2}+\frac{15}{4} g_{2}^{4}-\frac{2}{5}\left(-40 g_{3}^{2}+g_{1}^{2}\right) \operatorname{Tr}\left(Y_{d} Y_{d}^{\dagger}\right)+\frac{6}{5} g_{1}^{2} \operatorname{Tr}\left(Y_{e} Y_{e}^{\dagger}\right) \\
& -9 \operatorname{Tr}\left(Y_{d} Y_{d}^{\dagger} Y_{d} Y_{d}^{\dagger}\right)-3 \operatorname{Tr}\left(Y_{d} Y_{u}^{\dagger} Y_{u} Y_{d}^{\dagger}\right)-3 \operatorname{Tr}\left(Y_{e} Y_{e}^{\dagger} Y_{e} Y_{e}^{\dagger}\right) \\
\gamma_{\hat{H} u}^{(1)}= & -\frac{3}{10}\left(-10 \operatorname{Tr}\left(Y_{u} Y_{u}^{\dagger}\right)+5 g_{2}^{2}+g_{1}^{2}\right)
\end{aligned}
$$




$$
\begin{aligned}
& \gamma_{\hat{H}_{u}}^{(2)}=-3 \operatorname{Tr}\left(Y_{d} Y_{u}^{\dagger} Y_{u} Y_{d}^{\dagger}\right)-9 \operatorname{Tr}\left(Y_{u} Y_{u}^{\dagger} Y_{u} Y_{u}^{\dagger}\right)+\frac{15}{4} g_{2}^{4}+\frac{243}{100} g_{1}^{4} \\
&+\frac{4}{5}\left(20 g_{3}^{2}+g_{1}^{2}\right) \operatorname{Tr}\left(Y_{u} Y_{u}^{\dagger}\right)+\frac{9}{10} g_{1}^{2} g_{2}^{2} \\
& \gamma_{\hat{d}}^{(1)}= 2 Y_{d}^{*} Y_{d}^{T}-\frac{2}{15}\left(20 g_{3}^{2}+g_{1}^{2}\right) \mathbf{1} \\
& \gamma_{\hat{d}}^{(2)}=+\frac{2}{225}\left(-100 g_{3}^{4}+119 g_{1}^{4}+80 g_{1}^{2} g_{3}^{2}\right) \mathbf{1}-2\left(Y_{d}^{*} Y_{d}^{T} Y_{d}^{*} Y_{d}^{T}+Y_{d}^{*} Y_{u}^{T} Y_{u}^{*} Y_{d}^{T}\right) \\
&+Y_{d}^{*} Y_{d}^{T}\left(-2 \operatorname{Tr}\left(Y_{e} Y_{e}^{\dagger}\right)+6 g_{2}^{2}-6 \operatorname{Tr}\left(Y_{d} Y_{d}^{\dagger}\right)+\frac{2}{5} g_{1}^{2}\right) \\
& \gamma_{\hat{u}}^{(1)}= 2 Y_{u}^{*} Y_{u}^{T}-\frac{8}{15}\left(5 g_{3}^{2}+g_{1}^{2}\right) \mathbf{1} \\
& \gamma_{\hat{u}}^{(2)}=+\frac{8}{45}\left(16 g_{1}^{2} g_{3}^{2}+25 g_{1}^{4}-5 g_{3}^{4}\right) \mathbf{1}-2\left(Y_{u}^{*} Y_{d}^{T} Y_{d}^{*} Y_{u}^{T}+Y_{u}^{*} Y_{u}^{T} Y_{u}^{*} Y_{u}^{T}\right) \\
&+Y_{u}^{*} Y_{u}^{T}\left(6 g_{2}^{2}-6 \operatorname{Tr}\left(Y_{u} Y_{u}^{\dagger}\right)-\frac{2}{5} g_{1}^{2}\right) \\
& \gamma_{\hat{e}}^{(1)}= 2 Y_{e}^{*} Y_{e}^{T}-\frac{6}{5} g_{1}^{2} \mathbf{1} \\
& \gamma_{\hat{e}}^{(2)}=-2 Y_{e}^{*} Y_{e}^{T} Y_{e}^{*} Y_{e}^{T}+\frac{54}{5} g_{1}^{4} \mathbf{1}+Y_{e}^{*} Y_{e}^{T}\left(-2 \operatorname{Tr}\left(Y_{e} Y_{e}^{\dagger}\right)+6 g_{2}^{2}-6 \operatorname{Tr}\left(Y_{d} Y_{d}^{\dagger}\right)-\frac{6}{5} g_{1}^{2}\right) \\
& \gamma_{\phi_{F}^{+}}^{(1)}=-\frac{6}{5} g_{1}^{2}, \\
& \gamma_{\phi_{F}^{+}}^{(2)}=\frac{54}{5} g_{1}^{4}, \gamma_{\phi_{F}^{-}}^{(1)}=-\frac{6}{5} g_{1}^{2}, \\
& \gamma_{\phi_{F}}^{(2)}=\frac{54}{5} g_{1}^{4} .
\end{aligned}
$$

\section{A.2 Gauge couplings}

$$
\begin{aligned}
& \beta_{g_{1}}^{(1)}=\frac{39}{5} g_{1}^{3}, \quad \beta_{g_{2}}^{(1)}=g_{2}^{3} \quad, \quad \beta_{g_{3}}^{(1)}=-3 g_{3}^{3} \\
& \beta_{g_{1}}^{(2)}=\frac{1}{25} g_{1}^{3}\left(-130 \operatorname{Tr}\left(Y_{u} Y_{u}^{\dagger}\right)+135 g_{2}^{2}+271 g_{1}^{2}+440 g_{3}^{2}-70 \operatorname{Tr}\left(Y_{d} Y_{d}^{\dagger}\right)-90 \operatorname{Tr}\left(Y_{e} Y_{e}^{\dagger}\right)\right) \\
& \beta_{g_{2}}^{(2)}=\frac{1}{5} g_{2}^{3}\left(-10 \operatorname{Tr}\left(Y_{e} Y_{e}^{\dagger}\right)+120 g_{3}^{2}+125 g_{2}^{2}-30 \operatorname{Tr}\left(Y_{d} Y_{d}^{\dagger}\right)-30 \operatorname{Tr}\left(Y_{u} Y_{u}^{\dagger}\right)+9 g_{1}^{2}\right) \\
& \beta_{g_{3}}^{(2)}=\frac{1}{5} g_{3}^{3}\left(11 g_{1}^{2}-20 \operatorname{Tr}\left(Y_{d} Y_{d}^{\dagger}\right)-20 \operatorname{Tr}\left(Y_{u} Y_{u}^{\dagger}\right)+45 g_{2}^{2}+70 g_{3}^{2}\right)
\end{aligned}
$$

\section{A.3 Gaugino mass parameters}

$$
\begin{aligned}
\beta_{M_{1}}^{(1)}= & \frac{78}{5} g_{1}^{2} M_{1}, \quad \beta_{M_{2}}^{(1)}=2 g_{2}^{2} M_{2}, \quad \beta_{M_{3}}^{(1)}=-6 g_{3}^{2} M_{3} \\
\beta_{M_{1}}^{(2)}= & \frac{2}{25} g_{1}^{2}\left(542 g_{1}^{2} M_{1}+135 g_{2}^{2} M_{1}+440 g_{3}^{2} M_{1}+440 g_{3}^{2} M_{3}+135 g_{2}^{2} M_{2}-70 M_{1} \operatorname{Tr}\left(Y_{d} Y_{d}^{\dagger}\right)\right. \\
& \left.-90 M_{1} \operatorname{Tr}\left(Y_{e} Y_{e}^{\dagger}\right)-130 M_{1} \operatorname{Tr}\left(Y_{u} Y_{u}^{\dagger}\right)+70 \operatorname{Tr}\left(Y_{d}^{\dagger} T_{d}\right)+90 \operatorname{Tr}\left(Y_{e}^{\dagger} T_{e}\right)+130 \operatorname{Tr}\left(Y_{u}^{\dagger} T_{u}\right)\right)
\end{aligned}
$$




$$
\begin{aligned}
\beta_{M_{2}}^{(2)}= & \frac{2}{5} g_{2}^{2}\left(9 g_{1}^{2} M_{1}+120 g_{3}^{2} M_{3}+9 g_{1}^{2} M_{2}+250 g_{2}^{2} M_{2}+120 g_{3}^{2} M_{2}-30 M_{2} \operatorname{Tr}\left(Y_{d} Y_{d}^{\dagger}\right)\right. \\
& \left.-10 M_{2} \operatorname{Tr}\left(Y_{e} Y_{e}^{\dagger}\right)-30 M_{2} \operatorname{Tr}\left(Y_{u} Y_{u}^{\dagger}\right)+30 \operatorname{Tr}\left(Y_{d}^{\dagger} T_{d}\right)+10 \operatorname{Tr}\left(Y_{e}^{\dagger} T_{e}\right)+30 \operatorname{Tr}\left(Y_{u}^{\dagger} T_{u}\right)\right)
\end{aligned}
$$

$$
\begin{aligned}
\beta_{M_{3}}^{(2)}= & \frac{2}{5} g_{3}^{2}\left(11 g_{1}^{2} M_{1}+11 g_{1}^{2} M_{3}+45 g_{2}^{2} M_{3}+140 g_{3}^{2} M_{3}+45 g_{2}^{2} M_{2}-20 M_{3} \operatorname{Tr}\left(Y_{d} Y_{d}^{\dagger}\right)\right. \\
& \left.-20 M_{3} \operatorname{Tr}\left(Y_{u} Y_{u}^{\dagger}\right)+20 \operatorname{Tr}\left(Y_{d}^{\dagger} T_{d}\right)+20 \operatorname{Tr}\left(Y_{u}^{\dagger} T_{u}\right)\right)
\end{aligned}
$$

\section{A.4 Trilinear superpotential parameters}

$$
\begin{aligned}
\beta_{Y_{d}}^{(1)}= & 3 Y_{d} Y_{d}^{\dagger} Y_{d}+Y_{d}\left(-3 g_{2}^{2}+3 \operatorname{Tr}\left(Y_{d} Y_{d}^{\dagger}\right)-\frac{16}{3} g_{3}^{2}-\frac{7}{15} g_{1}^{2}+\operatorname{Tr}\left(Y_{e} Y_{e}^{\dagger}\right)\right)+Y_{d} Y_{u}^{\dagger} Y_{u} \\
\beta_{Y_{d}}^{(2)}= & +\frac{4}{5} g_{1}^{2} Y_{d} Y_{u}^{\dagger} Y_{u}-4 Y_{d} Y_{d}^{\dagger} Y_{d} Y_{d}^{\dagger} Y_{d}-2 Y_{d} Y_{u}^{\dagger} Y_{u} Y_{d}^{\dagger} Y_{d}-2 Y_{d} Y_{u}^{\dagger} Y_{u} Y_{u}^{\dagger} Y_{u} \\
& +Y_{d} Y_{d}^{\dagger} Y_{d}\left(-3 \operatorname{Tr}\left(Y_{e} Y_{e}^{\dagger}\right)+6 g_{2}^{2}-9 \operatorname{Tr}\left(Y_{d} Y_{d}^{\dagger}\right)+\frac{4}{5} g_{1}^{2}\right)-3 Y_{d} Y_{u}^{\dagger} Y_{u} \operatorname{Tr}\left(Y_{u} Y_{u}^{\dagger}\right) \\
& +Y_{d}\left(\frac{1687}{450} g_{1}^{4}+g_{1}^{2} g_{2}^{2}+\frac{15}{2} g_{2}^{4}+\frac{8}{9} g_{1}^{2} g_{3}^{2}+8 g_{2}^{2} g_{3}^{2}-\frac{16}{9} g_{3}^{4}-\frac{2}{5}\left(-40 g_{3}^{2}+g_{1}^{2}\right) \operatorname{Tr}\left(Y_{d} Y_{d}^{\dagger}\right)\right. \\
& \left.+\frac{6}{5} g_{1}^{2} \operatorname{Tr}\left(Y_{e} Y_{e}^{\dagger}\right)-9 \operatorname{Tr}\left(Y_{d} Y_{d}^{\dagger} Y_{d} Y_{d}^{\dagger}\right)-3 \operatorname{Tr}\left(Y_{d} Y_{u}^{\dagger} Y_{u} Y_{d}^{\dagger}\right)-3 \operatorname{Tr}\left(Y_{e} Y_{e}^{\dagger} Y_{e} Y_{e}^{\dagger}\right)\right) \\
\beta_{Y_{e}}^{(1)}= & 3 Y_{e} Y_{e}^{\dagger} Y_{e}+Y_{e}\left(-3 g_{2}^{2}+3 \operatorname{Tr}\left(Y_{d} Y_{d}^{\dagger}\right)-\frac{9}{5} g_{1}^{2}+\operatorname{Tr}\left(Y_{e} Y_{e}^{\dagger}\right)\right) \\
\beta_{Y_{e}}^{(2)}= & -4 Y_{e} Y_{e}^{\dagger} Y_{e} Y_{e}^{\dagger} Y_{e}+Y_{e} Y_{e}^{\dagger} Y_{e}\left(-3 \operatorname{Tr}\left(Y_{e} Y_{e}^{\dagger}\right)+6 g_{2}^{2}-9 \operatorname{Tr}\left(Y_{d} Y_{d}^{\dagger}\right)\right) \\
& +Y_{e}\left(\frac{783}{50} g_{1}^{4}+\frac{9}{5} g_{1}^{2} g_{2}^{2}+\frac{15}{2} g_{2}^{4}-\frac{2}{5}\left(-40 g_{3}^{2}+g_{1}^{2}\right) \operatorname{Tr}\left(Y_{d} Y_{d}^{\dagger}\right)+\frac{6}{5} g_{1}^{2} \operatorname{Tr}\left(Y_{e} Y_{e}^{\dagger}\right)\right. \\
& \left.-9 \operatorname{Tr}\left(Y_{d} Y_{d}^{\dagger} Y_{d} Y_{d}^{\dagger}\right)-3 \operatorname{Tr}\left(Y_{d} Y_{u}^{\dagger} Y_{u} Y_{d}^{\dagger}\right)-3 \operatorname{Tr}\left(Y_{e} Y_{e}^{\dagger} Y_{e} Y_{e}^{\dagger}\right)\right) \\
\beta_{Y_{u}}^{(1)}= & 3 Y_{u} Y_{u}^{\dagger} Y_{u}-\frac{1}{15} Y_{u}\left(13 g_{1}^{2}+45 g_{2}^{2}-45 \operatorname{Tr}\left(Y_{u} Y_{u}^{\dagger}\right)+80 g_{3}^{2}\right)+Y_{u} Y_{d}^{\dagger} Y_{d} \\
\beta_{Y_{u}}^{(2)}= & \frac{2}{5} g_{1}^{2} Y_{u} Y_{u}^{\dagger} Y_{u}+6 g_{2}^{2} Y_{u} Y_{u}^{\dagger} Y_{u}-2 Y_{u} Y_{d}^{\dagger} Y_{d} Y_{d}^{\dagger} Y_{d}-2 Y_{u} Y_{d}^{\dagger} Y_{d} Y_{u}^{\dagger} Y_{u} \\
& -4 Y_{u} Y_{u}^{\dagger} Y_{u} Y_{u}^{\dagger} Y_{u}+Y_{u} Y_{d}^{\dagger} Y_{d}\left(-3 \operatorname{Tr}\left(Y_{d} Y_{d}^{\dagger}\right)+\frac{2}{5} g_{1}^{2}-\operatorname{Tr}\left(Y_{e} Y_{e}^{\dagger}\right)\right)-9 g_{1}^{2} g_{2}^{2}+\frac{15}{2} g_{2}^{4}+\frac{136}{45} g_{1}^{2} g_{3}^{2}+8 g_{2}^{2} g_{3}^{2}-\frac{16}{9} g_{3}^{4}+\frac{4}{5}\left(20 g_{3}^{2}+g_{1}^{2}\right) \operatorname{Tr}\left(Y_{u} Y_{u}^{\dagger} Y_{u}^{\dagger}\right) \\
& -\mathrm{A} .26)
\end{aligned}
$$




\section{A.5 Bilinear superpotential parameters}

$$
\begin{aligned}
\beta_{\mu}^{(1)}= & 3 \mu \operatorname{Tr}\left(Y_{d} Y_{d}^{\dagger}\right)-\frac{3}{5} \mu\left(5 g_{2}^{2}-5 \operatorname{Tr}\left(Y_{u} Y_{u}^{\dagger}\right)+g_{1}^{2}\right)+\mu \operatorname{Tr}\left(Y_{e} Y_{e}^{\dagger}\right) \\
\beta_{\mu}^{(2)}= & \frac{1}{50} \mu\left(243 g_{1}^{4}+90 g_{1}^{2} g_{2}^{2}+375 g_{2}^{4}-20\left(-40 g_{3}^{2}+g_{1}^{2}\right) \operatorname{Tr}\left(Y_{d} Y_{d}^{\dagger}\right)+60 g_{1}^{2} \operatorname{Tr}\left(Y_{e} Y_{e}^{\dagger}\right)\right. \\
& +40 g_{1}^{2} \operatorname{Tr}\left(Y_{u} Y_{u}^{\dagger}\right)+800 g_{3}^{2} \operatorname{Tr}\left(Y_{u} Y_{u}^{\dagger}\right)-450 \operatorname{Tr}\left(Y_{d} Y_{d}^{\dagger} Y_{d} Y_{d}^{\dagger}\right)-300 \operatorname{Tr}\left(Y_{d} Y_{u}^{\dagger} Y_{u} Y_{d}^{\dagger}\right) \\
& \left.-150 \operatorname{Tr}\left(Y_{e} Y_{e}^{\dagger} Y_{e} Y_{e}^{\dagger}\right)-450 \operatorname{Tr}\left(Y_{u} Y_{u}^{\dagger} Y_{u} Y_{u}^{\dagger}\right)\right) \\
\beta_{\nu}^{(1)}= & -\frac{12}{5} g_{1}^{2} \nu, \quad \beta_{\nu}^{(2)}=\frac{108}{5} g_{1}^{4} \nu
\end{aligned}
$$

\section{A.6 Trilinear soft-breaking parameters}

$$
\begin{aligned}
& \beta_{T_{d}}^{(1)}=+4 Y_{d} Y_{d}^{\dagger} T_{d}+2 Y_{d} Y_{u}^{\dagger} T_{u}+5 T_{d} Y_{d}^{\dagger} Y_{d}+T_{d} Y_{u}^{\dagger} Y_{u}-\frac{7}{15} g_{1}^{2} T_{d}-3 g_{2}^{2} T_{d}-\frac{16}{3} g_{3}^{2} T_{d}+3 T_{d} \operatorname{Tr}\left(Y_{d} Y_{d}^{\dagger}\right) \\
& +T_{d} \operatorname{Tr}\left(Y_{e} Y_{e}^{\dagger}\right)+Y_{d}\left(2 \operatorname{Tr}\left(Y_{e}^{\dagger} T_{e}\right)+6 g_{2}^{2} M_{2}+6 \operatorname{Tr}\left(Y_{d}^{\dagger} T_{d}\right)+\frac{14}{15} g_{1}^{2} M_{1}+\frac{32}{3} g_{3}^{2} M_{3}\right) \\
& \beta_{T_{d}}^{(2)}=+\frac{6}{5} g_{1}^{2} Y_{d} Y_{d}^{\dagger} T_{d}+6 g_{2}^{2} Y_{d} Y_{d}^{\dagger} T_{d}-\frac{8}{5} g_{1}^{2} M_{1} Y_{d} Y_{u}^{\dagger} Y_{u}+\frac{8}{5} g_{1}^{2} Y_{d} Y_{u}^{\dagger} T_{u} \\
& +\frac{6}{5} g_{1}^{2} T_{d} Y_{d}^{\dagger} Y_{d}+12 g_{2}^{2} T_{d} Y_{d}^{\dagger} Y_{d}+\frac{4}{5} g_{1}^{2} T_{d} Y_{u}^{\dagger} Y_{u}-6 Y_{d} Y_{d}^{\dagger} Y_{d} Y_{d}^{\dagger} T_{d} \\
& -8 Y_{d} Y_{d}^{\dagger} T_{d} Y_{d}^{\dagger} Y_{d}-2 Y_{d} Y_{u}^{\dagger} Y_{u} Y_{d}^{\dagger} T_{d}-4 Y_{d} Y_{u}^{\dagger} Y_{u} Y_{u}^{\dagger} T_{u}-4 Y_{d} Y_{u}^{\dagger} T_{u} Y_{d}^{\dagger} Y_{d} \\
& -4 Y_{d} Y_{u}^{\dagger} T_{u} Y_{u}^{\dagger} Y_{u}-6 T_{d} Y_{d}^{\dagger} Y_{d} Y_{d}^{\dagger} Y_{d}-4 T_{d} Y_{u}^{\dagger} Y_{u} Y_{d}^{\dagger} Y_{d}-2 T_{d} Y_{u}^{\dagger} Y_{u} Y_{u}^{\dagger} Y_{u} \\
& +\frac{1687}{450} g_{1}^{4} T_{d}+g_{1}^{2} g_{2}^{2} T_{d}+\frac{15}{2} g_{2}^{4} T_{d}+\frac{8}{9} g_{1}^{2} g_{3}^{2} T_{d}+8 g_{2}^{2} g_{3}^{2} T_{d}-\frac{16}{9} g_{3}^{4} T_{d} \\
& -12 Y_{d} Y_{d}^{\dagger} T_{d} \operatorname{Tr}\left(Y_{d} Y_{d}^{\dagger}\right)-15 T_{d} Y_{d}^{\dagger} Y_{d} \operatorname{Tr}\left(Y_{d} Y_{d}^{\dagger}\right)-\frac{2}{5} g_{1}^{2} T_{d} \operatorname{Tr}\left(Y_{d} Y_{d}^{\dagger}\right) \\
& +16 g_{3}^{2} T_{d} \operatorname{Tr}\left(Y_{d} Y_{d}^{\dagger}\right)-4 Y_{d} Y_{d}^{\dagger} T_{d} \operatorname{Tr}\left(Y_{e} Y_{e}^{\dagger}\right)-5 T_{d} Y_{d}^{\dagger} Y_{d} \operatorname{Tr}\left(Y_{e} Y_{e}^{\dagger}\right) \\
& +\frac{6}{5} g_{1}^{2} T_{d} \operatorname{Tr}\left(Y_{e} Y_{e}^{\dagger}\right)-6 Y_{d} Y_{u}^{\dagger} T_{u} \operatorname{Tr}\left(Y_{u} Y_{u}^{\dagger}\right)-3 T_{d} Y_{u}^{\dagger} Y_{u} \operatorname{Tr}\left(Y_{u} Y_{u}^{\dagger}\right) \\
& -\frac{2}{5} Y_{d} Y_{d}^{\dagger} Y_{d}\left(15 \operatorname{Tr}\left(Y_{e}^{\dagger} T_{e}\right)+30 g_{2}^{2} M_{2}+45 \operatorname{Tr}\left(Y_{d}^{\dagger} T_{d}\right)+4 g_{1}^{2} M_{1}\right)-6 Y_{d} Y_{u}^{\dagger} Y_{u} \operatorname{Tr}\left(Y_{u}^{\dagger} T_{u}\right) \\
& -9 T_{d} \operatorname{Tr}\left(Y_{d} Y_{d}^{\dagger} Y_{d} Y_{d}^{\dagger}\right)-3 T_{d} \operatorname{Tr}\left(Y_{d} Y_{u}^{\dagger} Y_{u} Y_{d}^{\dagger}\right)-3 T_{d} \operatorname{Tr}\left(Y_{e} Y_{e}^{\dagger} Y_{e} Y_{e}^{\dagger}\right) \\
& +Y_{d}\left(-\frac{3374}{225} g_{1}^{4} M_{1}-2 g_{1}^{2} g_{2}^{2} M_{1}-\frac{16}{9} g_{1}^{2} g_{3}^{2} M_{1}-\frac{16}{9} g_{1}^{2} g_{3}^{2} M_{3}-16 g_{2}^{2} g_{3}^{2} M_{3}+\frac{64}{9} g_{3}^{4} M_{3}\right. \\
& -2 g_{1}^{2} g_{2}^{2} M_{2}-30 g_{2}^{4} M_{2}-16 g_{2}^{2} g_{3}^{2} M_{2}+\frac{4}{5}\left(-40 g_{3}^{2} M_{3}+g_{1}^{2} M_{1}\right) \operatorname{Tr}\left(Y_{d} Y_{d}^{\dagger}\right) \\
& -\frac{12}{5} g_{1}^{2} M_{1} \operatorname{Tr}\left(Y_{e} Y_{e}^{\dagger}\right)-\frac{4}{5} g_{1}^{2} \operatorname{Tr}\left(Y_{d}^{\dagger} T_{d}\right)+32 g_{3}^{2} \operatorname{Tr}\left(Y_{d}^{\dagger} T_{d}\right)+\frac{12}{5} g_{1}^{2} \operatorname{Tr}\left(Y_{e}^{\dagger} T_{e}\right) \\
& \left.-36 \operatorname{Tr}\left(Y_{d} Y_{d}^{\dagger} T_{d} Y_{d}^{\dagger}\right)-6 \operatorname{Tr}\left(Y_{d} Y_{u}^{\dagger} T_{u} Y_{d}^{\dagger}\right)-12 \operatorname{Tr}\left(Y_{e} Y_{e}^{\dagger} T_{e} Y_{e}^{\dagger}\right)-6 \operatorname{Tr}\left(Y_{u} Y_{d}^{\dagger} T_{d} Y_{u}^{\dagger}\right)\right)
\end{aligned}
$$




$$
\begin{aligned}
\beta_{T_{e}}^{(1)}= & +4 Y_{e} Y_{e}^{\dagger} T_{e}+5 T_{e} Y_{e}^{\dagger} Y_{e}-\frac{9}{5} g_{1}^{2} T_{e}-3 g_{2}^{2} T_{e}+3 T_{e} \operatorname{Tr}\left(Y_{d} Y_{d}^{\dagger}\right)+T_{e} \operatorname{Tr}\left(Y_{e} Y_{e}^{\dagger}\right) \\
& +Y_{e}\left(2 \operatorname{Tr}\left(Y_{e}^{\dagger} T_{e}\right)+6 g_{2}^{2} M_{2}+6 \operatorname{Tr}\left(Y_{d}^{\dagger} T_{d}\right)+\frac{18}{5} g_{1}^{2} M_{1}\right) \\
\beta_{T_{e}}^{(2)}= & +\frac{6}{5} g_{1}^{2} Y_{e} Y_{e}^{\dagger} T_{e}+6 g_{2}^{2} Y_{e} Y_{e}^{\dagger} T_{e}-\frac{6}{5} g_{1}^{2} T_{e} Y_{e}^{\dagger} Y_{e}+12 g_{2}^{2} T_{e} Y_{e}^{\dagger} Y_{e} \\
& -6 Y_{e} Y_{e}^{\dagger} Y_{e} Y_{e}^{\dagger} T_{e}-8 Y_{e} Y_{e}^{\dagger} T_{e} Y_{e}^{\dagger} Y_{e}-6 T_{e} Y_{e}^{\dagger} Y_{e} Y_{e}^{\dagger} Y_{e}+\frac{783}{50} g_{1}^{4} T_{e}+\frac{9}{5} g_{1}^{2} g_{2}^{2} T_{e}+\frac{15}{2} g_{2}^{4} T_{e} \\
& -12 Y_{e} Y_{e}^{\dagger} T_{e} \operatorname{Tr}\left(Y_{d} Y_{d}^{\dagger}\right)-15 T_{e} Y_{e}^{\dagger} Y_{e} \operatorname{Tr}\left(Y_{d} Y_{d}^{\dagger}\right)-\frac{2}{5} g_{1}^{2} T_{e} \operatorname{Tr}\left(Y_{d} Y_{d}^{\dagger}\right) \\
& +16 g_{3}^{2} T_{e} \operatorname{Tr}\left(Y_{d} Y_{d}^{\dagger}\right)-4 Y_{e} Y_{e}^{\dagger} T_{e} \operatorname{Tr}\left(Y_{e} Y_{e}^{\dagger}\right)-5 T_{e} Y_{e}^{\dagger} Y_{e} \operatorname{Tr}\left(Y_{e} Y_{e}^{\dagger}\right) \\
& +\frac{6}{5} g_{1}^{2} T_{e} \operatorname{Tr}\left(Y_{e} Y_{e}^{\dagger}\right)-6 Y_{e} Y_{e}^{\dagger} Y_{e}\left(2 g_{2}^{2} M_{2}+3 \operatorname{Tr}\left(Y_{d}^{\dagger} T_{d}\right)+\operatorname{Tr}\left(Y_{e}^{\dagger} T_{e}\right)\right)-9 T_{e} \operatorname{Tr}\left(Y_{d} Y_{d}^{\dagger} Y_{d} Y_{d}^{\dagger}\right) \\
& -3 T_{e} \operatorname{Tr}\left(Y_{d} Y_{u}^{\dagger} Y_{u} Y_{d}^{\dagger}\right)-3 T_{e} \operatorname{Tr}\left(Y_{e} Y_{e}^{\dagger} Y_{e} Y_{e}^{\dagger}\right) \\
& -\frac{2}{25} Y_{e}\left(783 g_{1}^{4} M_{1}+45 g_{1}^{2} g_{2}^{2} M_{1}+45 g_{1}^{2} g_{2}^{2} M_{2}+375 g_{2}^{4} M_{2}-10\left(-40 g_{3}^{2} M_{3}+g_{1}^{2} M_{1}\right) \operatorname{Tr}\left(Y_{d} Y_{d}^{\dagger}\right)\right. \\
& +30 g_{1}^{2} M_{1} \operatorname{Tr}\left(Y_{e} Y_{e}^{\dagger}\right)+10 g_{1}^{2} \operatorname{Tr}\left(Y_{d}^{\dagger} T_{d}\right)-400 g_{3}^{2} \operatorname{Tr}\left(Y_{d}^{\dagger} T_{d}\right)-30 g_{1}^{2} \operatorname{Tr}\left(Y_{e}^{\dagger} T_{e}\right) \\
& \left.+450 \operatorname{Tr}\left(Y_{d} Y_{d}^{\dagger} T_{d} Y_{d}^{\dagger}\right)+75 \operatorname{Tr}\left(Y_{d} Y_{u}^{\dagger} T_{u} Y_{d}^{\dagger}\right)+150 \operatorname{Tr}\left(Y_{e} Y_{e}^{\dagger} T_{e} Y_{e}^{\dagger}\right)+75 \operatorname{Tr}\left(Y_{u} Y_{d}^{\dagger} T_{d} Y_{u}^{\dagger}\right)\right)
\end{aligned}
$$

$$
\begin{aligned}
\beta_{T_{u}}^{(1)}= & +2 Y_{u} Y_{d}^{\dagger} T_{d}+4 Y_{u} Y_{u}^{\dagger} T_{u}+T_{u} Y_{d}^{\dagger} Y_{d}+5 T_{u} Y_{u}^{\dagger} Y_{u}-\frac{13}{15} g_{1}^{2} T_{u}-3 g_{2}^{2} T_{u}-\frac{16}{3} g_{3}^{2} T_{u} \\
& +3 T_{u} \operatorname{Tr}\left(Y_{u} Y_{u}^{\dagger}\right)+Y_{u}\left(6 g_{2}^{2} M_{2}+6 \operatorname{Tr}\left(Y_{u}^{\dagger} T_{u}\right)+\frac{26}{15} g_{1}^{2} M_{1}+\frac{32}{3} g_{3}^{2} M_{3}\right) \\
\beta_{T_{u}}^{(2)}= & +\frac{4}{5} g_{1}^{2} Y_{u} Y_{d}^{\dagger} T_{d}-\frac{4}{5} g_{1}^{2} M_{1} Y_{u} Y_{u}^{\dagger} Y_{u}-12 g_{2}^{2} M_{2} Y_{u} Y_{u}^{\dagger} Y_{u}+\frac{6}{5} g_{1}^{2} Y_{u} Y_{u}^{\dagger} T_{u}
\end{aligned}
$$$$
+6 g_{2}^{2} Y_{u} Y_{u}^{\dagger} T_{u}+\frac{2}{5} g_{1}^{2} T_{u} Y_{d}^{\dagger} Y_{d}+12 g_{2}^{2} T_{u} Y_{u}^{\dagger} Y_{u}-4 Y_{u} Y_{d}^{\dagger} Y_{d} Y_{d}^{\dagger} T_{d}
$$$$
-2 Y_{u} Y_{d}^{\dagger} Y_{d} Y_{u}^{\dagger} T_{u}-4 Y_{u} Y_{d}^{\dagger} T_{d} Y_{d}^{\dagger} Y_{d}-4 Y_{u} Y_{d}^{\dagger} T_{d} Y_{u}^{\dagger} Y_{u}-6 Y_{u} Y_{u}^{\dagger} Y_{u} Y_{u}^{\dagger} T_{u}
$$$$
-8 Y_{u} Y_{u}^{\dagger} T_{u} Y_{u}^{\dagger} Y_{u}-2 T_{u} Y_{d}^{\dagger} Y_{d} Y_{d}^{\dagger} Y_{d}-4 T_{u} Y_{d}^{\dagger} Y_{d} Y_{u}^{\dagger} Y_{u}-6 T_{u} Y_{u}^{\dagger} Y_{u} Y_{u}^{\dagger} Y_{u}+\frac{3211}{450} g_{1}^{4} T_{u}
$$$$
+g_{1}^{2} g_{2}^{2} T_{u}+\frac{15}{2} g_{2}^{4} T_{u}+\frac{136}{45} g_{1}^{2} g_{3}^{2} T_{u}+8 g_{2}^{2} g_{3}^{2} T_{u}-\frac{16}{9} g_{3}^{4} T_{u}-6 Y_{u} Y_{d}^{\dagger} T_{d} \operatorname{Tr}\left(Y_{d} Y_{d}^{\dagger}\right)
$$$$
-3 T_{u} Y_{d}^{\dagger} Y_{d} \operatorname{Tr}\left(Y_{d} Y_{d}^{\dagger}\right)-2 Y_{u} Y_{d}^{\dagger} T_{d} \operatorname{Tr}\left(Y_{e} Y_{e}^{\dagger}\right)-T_{u} Y_{d}^{\dagger} Y_{d} \operatorname{Tr}\left(Y_{e} Y_{e}^{\dagger}\right)
$$$$
-12 Y_{u} Y_{u}^{\dagger} T_{u} \operatorname{Tr}\left(Y_{u} Y_{u}^{\dagger}\right)-15 T_{u} Y_{u}^{\dagger} Y_{u} \operatorname{Tr}\left(Y_{u} Y_{u}^{\dagger}\right)+\frac{4}{5} g_{1}^{2} T_{u} \operatorname{Tr}\left(Y_{u} Y_{u}^{\dagger}\right)
$$$$
+16 g_{3}^{2} T_{u} \operatorname{Tr}\left(Y_{u} Y_{u}^{\dagger}\right)-\frac{2}{5} Y_{u} Y_{d}^{\dagger} Y_{d}\left(15 \operatorname{Tr}\left(Y_{d}^{\dagger} T_{d}\right)+2 g_{1}^{2} M_{1}+5 \operatorname{Tr}\left(Y_{e}^{\dagger} T_{e}\right)\right)
$$$$
-18 Y_{u} Y_{u}^{\dagger} Y_{u} \operatorname{Tr}\left(Y_{u}^{\dagger} T_{u}\right)-3 T_{u} \operatorname{Tr}\left(Y_{d} Y_{u}^{\dagger} Y_{u} Y_{d}^{\dagger}\right)-9 T_{u} \operatorname{Tr}\left(Y_{u} Y_{u}^{\dagger} Y_{u} Y_{u}^{\dagger}\right)
$$$$
-\frac{2}{225} Y_{u}\left(3211 g_{1}^{4} M_{1}+225 g_{1}^{2} g_{2}^{2} M_{1}+680 g_{1}^{2} g_{3}^{2} M_{1}+680 g_{1}^{2} g_{3}^{2} M_{3}+1800 g_{2}^{2} g_{3}^{2} M_{3}-800 g_{3}^{4} M_{3}\right.
$$ 


$$
\begin{aligned}
& +225 g_{1}^{2} g_{2}^{2} M_{2}+3375 g_{2}^{4} M_{2}+1800 g_{2}^{2} g_{3}^{2} M_{2}+180\left(20 g_{3}^{2} M_{3}+g_{1}^{2} M_{1}\right) \operatorname{Tr}\left(Y_{u} Y_{u}^{\dagger}\right) \\
& -180\left(20 g_{3}^{2}+g_{1}^{2}\right) \operatorname{Tr}\left(Y_{u}^{\dagger} T_{u}\right)+675 \operatorname{Tr}\left(Y_{d} Y_{u}^{\dagger} T_{u} Y_{d}^{\dagger}\right)+675 \operatorname{Tr}\left(Y_{u} Y_{d}^{\dagger} T_{d} Y_{u}^{\dagger}\right) \\
& \left.+4050 \operatorname{Tr}\left(Y_{u} Y_{u}^{\dagger} T_{u} Y_{u}^{\dagger}\right)\right)
\end{aligned}
$$

\section{A.7 Vacuum expectation values}

$$
\begin{aligned}
\beta_{v_{d}}^{(1)}= & \frac{1}{20} v_{d}\left(-20 \operatorname{Tr}\left(Y_{e} Y_{e}^{\dagger}\right)+3\left(5 g_{2}^{2}+g_{1}^{2}\right)(1+\xi)-60 \operatorname{Tr}\left(Y_{d} Y_{d}^{\dagger}\right)\right) \\
\beta_{v_{d}}^{(2)}= & \frac{1}{400} v_{d}\left(-486 g_{1}^{4}-180 g_{1}^{2} g_{2}^{2}-1200 g_{2}^{4}-9 g_{1}^{4} \xi-90 g_{1}^{2} g_{2}^{2} \xi+875 g_{2}^{4} \xi+9 g_{1}^{4} \xi^{2}+90 g_{1}^{2} g_{2}^{2} \xi^{2}\right. \\
& -225 g_{2}^{4} \xi^{2}-40\left(5\left(32 g_{3}^{2}+9 g_{2}^{2} \xi\right)+g_{1}^{2}(9 \xi-4)\right) \operatorname{Tr}\left(Y_{d} Y_{d}^{\dagger}\right) \\
& -120\left(5 g_{2}^{2} \xi+g_{1}^{2}(4+\xi)\right) \operatorname{Tr}\left(Y_{e} Y_{e}^{\dagger}\right) \\
& \left.+3600 \operatorname{Tr}\left(Y_{d} Y_{d}^{\dagger} Y_{d} Y_{d}^{\dagger}\right)+1200 \operatorname{Tr}\left(Y_{d} Y_{u}^{\dagger} Y_{u} Y_{d}^{\dagger}\right)+1200 \operatorname{Tr}\left(Y_{e} Y_{e}^{\dagger} Y_{e} Y_{e}^{\dagger}\right)\right) \\
\beta_{v_{u}}^{(1)}= & \frac{3}{20} v_{u}\left(-20 \operatorname{Tr}\left(Y_{u} Y_{u}^{\dagger}\right)+\left(5 g_{2}^{2}+g_{1}^{2}\right)(1+\xi)\right) \\
\beta_{v_{u}}^{(2)}= & \frac{1}{400} v_{u}\left(-486 g_{1}^{4}-180 g_{1}^{2} g_{2}^{2}-1200 g_{2}^{4}-9 g_{1}^{4} \xi-90 g_{1}^{2} g_{2}^{2} \xi+875 g_{2}^{4} \xi+9 g_{1}^{4} \xi^{2}+90 g_{1}^{2} g_{2}^{2} \xi^{2}\right. \\
& -225 g_{2}^{4} \xi^{2}-40\left(5\left(32 g_{3}^{2}+9 g_{2}^{2} \xi\right)+g_{1}^{2}(9 \xi+8)\right) \operatorname{Tr}\left(Y_{u} Y_{u}^{\dagger}\right) \\
& \left.+1200 \operatorname{Tr}\left(Y_{d} Y_{u}^{\dagger} Y_{u} Y_{d}^{\dagger}\right)+3600 \operatorname{Tr}\left(Y_{u} Y_{u}^{\dagger} Y_{u} Y_{u}^{\dagger}\right)\right)
\end{aligned}
$$

Note that $\xi$ is the gauge-fixing parameter, where we are using the $R_{\xi}$ gauge.

\section{A.8 Bilinear soft-breaking parameters}

$$
\begin{aligned}
\beta_{B_{\mu}}^{(1)}= & +\frac{6}{5} g_{1}^{2} M_{1} \mu+6 g_{2}^{2} M_{2} \mu+B_{\mu}\left(-3 g_{2}^{2}+3 \operatorname{Tr}\left(Y_{d} Y_{d}^{\dagger}\right)+3 \operatorname{Tr}\left(Y_{u} Y_{u}^{\dagger}\right)-\frac{3}{5} g_{1}^{2}+\operatorname{Tr}\left(Y_{e} Y_{e}^{\dagger}\right)\right) \\
& +6 \mu \operatorname{Tr}\left(Y_{d}^{\dagger} T_{d}\right)+2 \mu \operatorname{Tr}\left(Y_{e}^{\dagger} T_{e}\right)+6 \mu \operatorname{Tr}\left(Y_{u}^{\dagger} T_{u}\right) \\
\beta_{B_{\mu}}^{(2)}= & B_{\mu}\left(\frac{243}{50} g_{1}^{4}+\frac{9}{5} g_{1}^{2} g_{2}^{2}+\frac{15}{2} g_{2}^{4}-\frac{2}{5}\left(-40 g_{3}^{2}+g_{1}^{2}\right) \operatorname{Tr}\left(Y_{d} Y_{d}^{\dagger}\right)+\frac{6}{5} g_{1}^{2} \operatorname{Tr}\left(Y_{e} Y_{e}^{\dagger}\right)+\frac{4}{5} g_{1}^{2} \operatorname{Tr}\left(Y_{u} Y_{u}^{\dagger}\right)\right. \\
& \left.+16 g_{3}^{2} \operatorname{Tr}\left(Y_{u} Y_{u}^{\dagger}\right)-9 \operatorname{Tr}\left(Y_{d} Y_{d}^{\dagger} Y_{d} Y_{d}^{\dagger}\right)-6 \operatorname{Tr}\left(Y_{d} Y_{u}^{\dagger} Y_{u} Y_{d}^{\dagger}\right)-3 \operatorname{Tr}\left(Y_{e} Y_{e}^{\dagger} Y_{e} Y_{e}^{\dagger}\right)-9 \operatorname{Tr}\left(Y_{u} Y_{u}^{\dagger} Y_{u} Y_{u}^{\dagger}\right)\right) \\
& -\frac{2}{25} \mu\left(243 g_{1}^{4} M_{1}+45 g_{1}^{2} g_{2}^{2} M_{1}+45 g_{1}^{2} g_{2}^{2} M_{2}+375 g_{2}^{4} M_{2}-10\left(-40 g_{3}^{2} M_{3}+g_{1}^{2} M_{1}\right) \operatorname{Tr}\left(Y_{d} Y_{d}^{\dagger}\right)\right. \\
& +30 g_{1}^{2} M_{1} \operatorname{Tr}\left(Y_{e} Y_{e}^{\dagger}\right)+20 g_{1}^{2} M_{1} \operatorname{Tr}\left(Y_{u} Y_{u}^{\dagger}\right)+400 g_{3}^{2} M_{3} \operatorname{Tr}\left(Y_{u} Y_{u}^{\dagger}\right)+10 g_{1}^{2} \operatorname{Tr}\left(Y_{d}^{\dagger} T_{d}\right) \\
& -400 g_{3}^{2} \operatorname{Tr}\left(Y_{d}^{\dagger} T_{d}\right)-30 g_{1}^{2} \operatorname{Tr}\left(Y_{e}^{\dagger} T_{e}\right)-20 g_{1}^{2} \operatorname{Tr}\left(Y_{u}^{\dagger} T_{u}\right)-400 g_{3}^{2} \operatorname{Tr}\left(Y_{u}^{\dagger} T_{u}\right)
\end{aligned}
$$




$$
\begin{aligned}
& +450 \operatorname{Tr}\left(Y_{d} Y_{d}^{\dagger} T_{d} Y_{d}^{\dagger}\right)+150 \operatorname{Tr}\left(Y_{d} Y_{u}^{\dagger} T_{u} Y_{d}^{\dagger}\right)+150 \operatorname{Tr}\left(Y_{e} Y_{e}^{\dagger} T_{e} Y_{e}^{\dagger}\right)+150 \operatorname{Tr}\left(Y_{u} Y_{d}^{\dagger} T_{d} Y_{u}^{\dagger}\right) \\
& \left.+450 \operatorname{Tr}\left(Y_{u} Y_{u}^{\dagger} T_{u} Y_{u}^{\dagger}\right)\right) \\
\beta_{B_{\nu}}^{(1)}= & \frac{12}{5} g_{1}^{2}\left(2 M_{1} \nu-B_{\nu}\right), \quad \beta_{B_{\nu}}^{(2)}=-\frac{108}{5} g_{1}^{4}\left(4 M_{1} \nu-B_{\nu}\right) .
\end{aligned}
$$

\section{A.9 Soft-breaking scalar masses}

$$
\begin{aligned}
& \sigma_{1,1}=\sqrt{\frac{3}{5}} g_{1}\left(-2 \operatorname{Tr}\left(m_{u}^{2}\right)-\operatorname{Tr}\left(m_{l}^{2}\right)-m_{H_{d}}^{2}-m_{F_{-}}^{2}+m_{H_{u}}^{2}+m_{F_{+}}^{2}+\operatorname{Tr}\left(m_{d}^{2}\right)\right. \\
& \left.+\operatorname{Tr}\left(m_{e}^{2}\right)+\operatorname{Tr}\left(m_{q}^{2}\right)\right) \\
& \sigma_{2,11}=\frac{1}{10} g_{1}^{2}\left(2 \operatorname{Tr}\left(m_{d}^{2}\right)+3 \operatorname{Tr}\left(m_{l}^{2}\right)+3 m_{H_{d}}^{2}+3 m_{H_{u}}^{2}+6 \operatorname{Tr}\left(m_{e}^{2}\right)+6 m_{F^{-}}^{2}+6 m_{F^{+}}^{2}\right. \\
& \left.+8 \operatorname{Tr}\left(m_{u}^{2}\right)+\operatorname{Tr}\left(m_{q}^{2}\right)\right) \\
& \sigma_{3,1}=\frac{1}{20} \frac{1}{\sqrt{15}} g_{1}\left(-9 g_{1}^{2} m_{H_{d}}^{2}-45 g_{2}^{2} m_{H_{d}}^{2}+9 g_{1}^{2} m_{H_{u}}^{2}+45 g_{2}^{2} m_{H_{u}}^{2}-36 g_{1}^{2} m_{F^{-}}^{2}+36 g_{1}^{2} m_{F^{+}}^{2}\right. \\
& +4\left(20 g_{3}^{2}+g_{1}^{2}\right) \operatorname{Tr}\left(m_{d}^{2}\right)+36 g_{1}^{2} \operatorname{Tr}\left(m_{e}^{2}\right)-9 g_{1}^{2} \operatorname{Tr}\left(m_{l}^{2}\right)-45 g_{2}^{2} \operatorname{Tr}\left(m_{l}^{2}\right)+g_{1}^{2} \operatorname{Tr}\left(m_{q}^{2}\right) \\
& +45 g_{2}^{2} \operatorname{Tr}\left(m_{q}^{2}\right)+80 g_{3}^{2} \operatorname{Tr}\left(m_{q}^{2}\right)-32 g_{1}^{2} \operatorname{Tr}\left(m_{u}^{2}\right)-160 g_{3}^{2} \operatorname{Tr}\left(m_{u}^{2}\right)+90 m_{H_{d}}^{2} \operatorname{Tr}\left(Y_{d} Y_{d}^{\dagger}\right) \\
& +30 m_{H_{d}}^{2} \operatorname{Tr}\left(Y_{e} Y_{e}^{\dagger}\right)-90 m_{H_{u}}^{2} \operatorname{Tr}\left(Y_{u} Y_{u}^{\dagger}\right)-60 \operatorname{Tr}\left(Y_{d} Y_{d}^{\dagger} m_{d}^{2 *}\right)-30 \operatorname{Tr}\left(Y_{d} m_{q}^{2 *} Y_{d}^{\dagger}\right) \\
& \left.-60 \operatorname{Tr}\left(Y_{e} Y_{e}^{\dagger} m_{e}^{2 *}\right)+30 \operatorname{Tr}\left(Y_{e} m_{l}^{2 *} Y_{e}^{\dagger}\right)+120 \operatorname{Tr}\left(Y_{u} Y_{u}^{\dagger} m_{u}^{2 *}\right)-30 \operatorname{Tr}\left(Y_{u} m_{q}^{2 *} Y_{u}^{\dagger}\right)\right) \\
& \sigma_{2,2}=\frac{1}{2}\left(3 \operatorname{Tr}\left(m_{q}^{2}\right)+m_{H_{d}}^{2}+m_{H_{u}}^{2}+\operatorname{Tr}\left(m_{l}^{2}\right)\right) \\
& \sigma_{2,3}=\frac{1}{2}\left(2 \operatorname{Tr}\left(m_{q}^{2}\right)+\operatorname{Tr}\left(m_{d}^{2}\right)+\operatorname{Tr}\left(m_{u}^{2}\right)\right) \\
& \beta_{m_{q}^{2}}^{(1)}=-\frac{2}{15} g_{1}^{2} \mathbf{1}\left|M_{1}\right|^{2}-\frac{32}{3} g_{3}^{2} \mathbf{1}\left|M_{3}\right|^{2}-6 g_{2}^{2} \mathbf{1}\left|M_{2}\right|^{2}+2 m_{H_{d}}^{2} Y_{d}^{\dagger} Y_{d}+2 m_{H_{u}}^{2} Y_{u}^{\dagger} Y_{u}+2 T_{d}^{\dagger} T_{d} \\
& +2 T_{u}^{\dagger} T_{u}+m_{q}^{2} Y_{d}^{\dagger} Y_{d}+m_{q}^{2} Y_{u}^{\dagger} Y_{u}+2 Y_{d}^{\dagger} m_{d}^{2} Y_{d}+Y_{d}^{\dagger} Y_{d} m_{q}^{2}+2 Y_{u}^{\dagger} m_{u}^{2} Y_{u} \\
& +Y_{u}^{\dagger} Y_{u} m_{q}^{2}+\frac{1}{\sqrt{15}} g_{1} 1 \sigma_{1,1} \\
& \beta_{m_{q}^{2}}^{(2)}=+\frac{2}{5} g_{1}^{2} g_{2}^{2} \mathbf{1}\left|M_{2}\right|^{2}+33 g_{2}^{4} \mathbf{1}\left|M_{2}\right|^{2}+32 g_{2}^{2} g_{3}^{2} \mathbf{1}\left|M_{2}\right|^{2} \\
& +\frac{16}{45} g_{3}^{2}\left(15\left(3 g_{2}^{2}\left(2 M_{3}+M_{2}\right)-8 g_{3}^{2} M_{3}\right)+g_{1}^{2}\left(2 M_{3}+M_{1}\right)\right) 1 M_{3}^{*} \\
& +\frac{1}{5} g_{1}^{2} g_{2}^{2} M_{1} \mathbf{1} M_{2}^{*}+16 g_{2}^{2} g_{3}^{2} M_{3} \mathbf{1} M_{2}^{*}+\frac{4}{5} g_{1}^{2} m_{H_{d}}^{2} Y_{d}^{\dagger} Y_{d}+\frac{8}{5} g_{1}^{2} m_{H_{u}}^{2} Y_{u}^{\dagger} Y_{u} \\
& +\frac{1}{45} g_{1}^{2} M_{1}^{*}\left(\left(141 g_{1}^{2} M_{1}+16 g_{3}^{2}\left(2 M_{1}+M_{3}\right)+9 g_{2}^{2}\left(2 M_{1}+M_{2}\right)\right) \mathbf{1}\right. \\
& \left.+36\left(2 M_{1} Y_{d}^{\dagger} Y_{d}-2 Y_{u}^{\dagger} T_{u}+4 M_{1} Y_{u}^{\dagger} Y_{u}-Y_{d}^{\dagger} T_{d}\right)\right) \\
& -\frac{4}{5} g_{1}^{2} M_{1} T_{d}^{\dagger} Y_{d}+\frac{4}{5} g_{1}^{2} T_{d}^{\dagger} T_{d}-\frac{8}{5} g_{1}^{2} M_{1} T_{u}^{\dagger} Y_{u}+\frac{8}{5} g_{1}^{2} T_{u}^{\dagger} T_{u} \\
& +\frac{2}{5} g_{1}^{2} m_{q}^{2} Y_{d}^{\dagger} Y_{d}+\frac{4}{5} g_{1}^{2} m_{q}^{2} Y_{u}^{\dagger} Y_{u}+\frac{4}{5} g_{1}^{2} Y_{d}^{\dagger} m_{d}^{2} Y_{d}+\frac{2}{5} g_{1}^{2} Y_{d}^{\dagger} Y_{d} m_{q}^{2} \\
& +\frac{8}{5} g_{1}^{2} Y_{u}^{\dagger} m_{u}^{2} Y_{u}+\frac{4}{5} g_{1}^{2} Y_{u}^{\dagger} Y_{u} m_{q}^{2}-8 m_{H_{d}}^{2} Y_{d}^{\dagger} Y_{d} Y_{d}^{\dagger} Y_{d}-4 Y_{d}^{\dagger} Y_{d} T_{d}^{\dagger} T_{d}
\end{aligned}
$$




$$
\begin{aligned}
& -4 Y_{d}^{\dagger} T_{d} T_{d}^{\dagger} Y_{d}-8 m_{H_{u}}^{2} Y_{u}^{\dagger} Y_{u} Y_{u}^{\dagger} Y_{u}-4 Y_{u}^{\dagger} Y_{u} T_{u}^{\dagger} T_{u}-4 Y_{u}^{\dagger} T_{u} T_{u}^{\dagger} Y_{u} \\
& -4 T_{d}^{\dagger} Y_{d} Y_{d}^{\dagger} T_{d}-4 T_{d}^{\dagger} T_{d} Y_{d}^{\dagger} Y_{d}-4 T_{u}^{\dagger} Y_{u} Y_{u}^{\dagger} T_{u}-4 T_{u}^{\dagger} T_{u} Y_{u}^{\dagger} Y_{u} \\
& -2 m_{q}^{2} Y_{d}^{\dagger} Y_{d} Y_{d}^{\dagger} Y_{d}-2 m_{q}^{2} Y_{u}^{\dagger} Y_{u} Y_{u}^{\dagger} Y_{u}-4 Y_{d}^{\dagger} m_{d}^{2} Y_{d} Y_{d}^{\dagger} Y_{d}-4 Y_{d}^{\dagger} Y_{d} m_{q}^{2} Y_{d}^{\dagger} Y_{d} \\
& -4 Y_{d}^{\dagger} Y_{d} Y_{d}^{\dagger} m_{d}^{2} Y_{d}-2 Y_{d}^{\dagger} Y_{d} Y_{d}^{\dagger} Y_{d} m_{q}^{2}-4 Y_{u}^{\dagger} m_{u}^{2} Y_{u} Y_{u}^{\dagger} Y_{u}-4 Y_{u}^{\dagger} Y_{u} m_{q}^{2} Y_{u}^{\dagger} Y_{u} \\
& -4 Y_{u}^{\dagger} Y_{u} Y_{u}^{\dagger} m_{u}^{2} Y_{u}-2 Y_{u}^{\dagger} Y_{u} Y_{u}^{\dagger} Y_{u} m_{q}^{2}+6 g_{2}^{4} \mathbf{1} \sigma_{2,2}+\frac{32}{3} g_{3}^{4} \mathbf{1} \sigma_{2,3}+\frac{2}{15} g_{1}^{2} \mathbf{1} \sigma_{2,11}+4 \frac{1}{\sqrt{15}} g_{1} \mathbf{1} \sigma_{3,1} \\
& -12 m_{H_{d}}^{2} Y_{d}^{\dagger} Y_{d} \operatorname{Tr}\left(Y_{d} Y_{d}^{\dagger}\right)-6 T_{d}^{\dagger} T_{d} \operatorname{Tr}\left(Y_{d} Y_{d}^{\dagger}\right)-3 m_{q}^{2} Y_{d}^{\dagger} Y_{d} \operatorname{Tr}\left(Y_{d} Y_{d}^{\dagger}\right) \\
& -6 Y_{d}^{\dagger} m_{d}^{2} Y_{d} \operatorname{Tr}\left(Y_{d} Y_{d}^{\dagger}\right)-3 Y_{d}^{\dagger} Y_{d} m_{q}^{2} \operatorname{Tr}\left(Y_{d} Y_{d}^{\dagger}\right)-4 m_{H_{d}}^{2} Y_{d}^{\dagger} Y_{d} \operatorname{Tr}\left(Y_{e} Y_{e}^{\dagger}\right) \\
& -2 T_{d}^{\dagger} T_{d} \operatorname{Tr}\left(Y_{e} Y_{e}^{\dagger}\right)-m_{q}^{2} Y_{d}^{\dagger} Y_{d} \operatorname{Tr}\left(Y_{e} Y_{e}^{\dagger}\right)-2 Y_{d}^{\dagger} m_{d}^{2} Y_{d} \operatorname{Tr}\left(Y_{e} Y_{e}^{\dagger}\right) \\
& -Y_{d}^{\dagger} Y_{d} m_{q}^{2} \operatorname{Tr}\left(Y_{e} Y_{e}^{\dagger}\right)-12 m_{H_{u}}^{2} Y_{u}^{\dagger} Y_{u} \operatorname{Tr}\left(Y_{u} Y_{u}^{\dagger}\right)-6 T_{u}^{\dagger} T_{u} \operatorname{Tr}\left(Y_{u} Y_{u}^{\dagger}\right) \\
& -3 m_{q}^{2} Y_{u}^{\dagger} Y_{u} \operatorname{Tr}\left(Y_{u} Y_{u}^{\dagger}\right)-6 Y_{u}^{\dagger} m_{u}^{2} Y_{u} \operatorname{Tr}\left(Y_{u} Y_{u}^{\dagger}\right)-3 Y_{u}^{\dagger} Y_{u} m_{q}^{2} \operatorname{Tr}\left(Y_{u} Y_{u}^{\dagger}\right) \\
& -6 T_{d}^{\dagger} Y_{d} \operatorname{Tr}\left(Y_{d}^{\dagger} T_{d}\right)-2 T_{d}^{\dagger} Y_{d} \operatorname{Tr}\left(Y_{e}^{\dagger} T_{e}\right)-6 T_{u}^{\dagger} Y_{u} \operatorname{Tr}\left(Y_{u}^{\dagger} T_{u}\right) \\
& -6 Y_{d}^{\dagger} T_{d} \operatorname{Tr}\left(T_{d}^{*} Y_{d}^{T}\right)-6 Y_{d}^{\dagger} Y_{d} \operatorname{Tr}\left(T_{d}^{*} T_{d}^{T}\right)-2 Y_{d}^{\dagger} T_{d} \operatorname{Tr}\left(T_{e}^{*} Y_{e}^{T}\right) \\
& -2 Y_{d}^{\dagger} Y_{d} \operatorname{Tr}\left(T_{e}^{*} T_{e}^{T}\right)-6 Y_{u}^{\dagger} T_{u} \operatorname{Tr}\left(T_{u}^{*} Y_{u}^{T}\right)-6 Y_{u}^{\dagger} Y_{u} \operatorname{Tr}\left(T_{u}^{*} T_{u}^{T}\right) \\
& -6 Y_{d}^{\dagger} Y_{d} \operatorname{Tr}\left(m_{d}^{2} Y_{d} Y_{d}^{\dagger}\right)-2 Y_{d}^{\dagger} Y_{d} \operatorname{Tr}\left(m_{e}^{2} Y_{e} Y_{e}^{\dagger}\right)-2 Y_{d}^{\dagger} Y_{d} \operatorname{Tr}\left(m_{l}^{2} Y_{e}^{\dagger} Y_{e}\right) \\
& -6 Y_{d}^{\dagger} Y_{d} \operatorname{Tr}\left(m_{q}^{2} Y_{d}^{\dagger} Y_{d}\right)-6 Y_{u}^{\dagger} Y_{u} \operatorname{Tr}\left(m_{q}^{2} Y_{u}^{\dagger} Y_{u}\right)-6 Y_{u}^{\dagger} Y_{u} \operatorname{Tr}\left(m_{u}^{2} Y_{u} Y_{u}^{\dagger}\right) \\
& \beta_{m_{l}^{2}}^{(1)}=-\frac{6}{5} g_{1}^{2} \mathbf{1}\left|M_{1}\right|^{2}-6 g_{2}^{2} \mathbf{1}\left|M_{2}\right|^{2}+2 m_{H_{d}}^{2} Y_{e}^{\dagger} Y_{e}+2 T_{e}^{\dagger} T_{e}+m_{l}^{2} Y_{e}^{\dagger} Y_{e}+2 Y_{e}^{\dagger} m_{e}^{2} Y_{e} \\
& +Y_{e}^{\dagger} Y_{e} m_{l}^{2}-\sqrt{\frac{3}{5}} g_{1} \mathbf{1} \sigma_{1,1} \\
& \beta_{m_{l}^{2}}^{(2)}=+\frac{3}{5} g_{2}^{2}\left(3 g_{1}^{2}\left(2 M_{2}+M_{1}\right)+55 g_{2}^{2} M_{2}\right) \mathbf{1} M_{2}^{*}+\frac{12}{5} g_{1}^{2} m_{H_{d}}^{2} Y_{e}^{\dagger} Y_{e} \\
& +\frac{3}{25} g_{1}^{2} M_{1}^{*}\left(-20 Y_{e}^{\dagger} T_{e}+3\left(5 g_{2}^{2}\left(2 M_{1}+M_{2}\right)+81 g_{1}^{2} M_{1}\right) \mathbf{1}+40 M_{1} Y_{e}^{\dagger} Y_{e}\right)-\frac{12}{5} g_{1}^{2} M_{1} T_{e}^{\dagger} Y_{e} \\
& +\frac{12}{5} g_{1}^{2} T_{e}^{\dagger} T_{e}+\frac{6}{5} g_{1}^{2} m_{l}^{2} Y_{e}^{\dagger} Y_{e}+\frac{12}{5} g_{1}^{2} Y_{e}^{\dagger} m_{e}^{2} Y_{e}+\frac{6}{5} g_{1}^{2} Y_{e}^{\dagger} Y_{e} m_{l}^{2} \\
& -8 m_{H_{d}}^{2} Y_{e}^{\dagger} Y_{e} Y_{e}^{\dagger} Y_{e}-4 Y_{e}^{\dagger} Y_{e} T_{e}^{\dagger} T_{e}-4 Y_{e}^{\dagger} T_{e} T_{e}^{\dagger} Y_{e}-4 T_{e}^{\dagger} Y_{e} Y_{e}^{\dagger} T_{e} \\
& -4 T_{e}^{\dagger} T_{e} Y_{e}^{\dagger} Y_{e}-2 m_{l}^{2} Y_{e}^{\dagger} Y_{e} Y_{e}^{\dagger} Y_{e}-4 Y_{e}^{\dagger} m_{e}^{2} Y_{e} Y_{e}^{\dagger} Y_{e}-4 Y_{e}^{\dagger} Y_{e} m_{l}^{2} Y_{e}^{\dagger} Y_{e} \\
& -4 Y_{e}^{\dagger} Y_{e} Y_{e}^{\dagger} m_{e}^{2} Y_{e}-2 Y_{e}^{\dagger} Y_{e} Y_{e}^{\dagger} Y_{e} m_{l}^{2}+6 g_{2}^{4} \mathbf{1} \sigma_{2,2}+\frac{6}{5} g_{1}^{2} \mathbf{1} \sigma_{2,11}-4 \sqrt{\frac{3}{5}} g_{1} \mathbf{1} \sigma_{3,1} \\
& -12 m_{H_{d}}^{2} Y_{e}^{\dagger} Y_{e} \operatorname{Tr}\left(Y_{d} Y_{d}^{\dagger}\right)-6 T_{e}^{\dagger} T_{e} \operatorname{Tr}\left(Y_{d} Y_{d}^{\dagger}\right)-3 m_{l}^{2} Y_{e}^{\dagger} Y_{e} \operatorname{Tr}\left(Y_{d} Y_{d}^{\dagger}\right) \\
& -6 Y_{e}^{\dagger} m_{e}^{2} Y_{e} \operatorname{Tr}\left(Y_{d} Y_{d}^{\dagger}\right)-3 Y_{e}^{\dagger} Y_{e} m_{l}^{2} \operatorname{Tr}\left(Y_{d} Y_{d}^{\dagger}\right)-4 m_{H_{d}}^{2} Y_{e}^{\dagger} Y_{e} \operatorname{Tr}\left(Y_{e} Y_{e}^{\dagger}\right) \\
& -2 T_{e}^{\dagger} T_{e} \operatorname{Tr}\left(Y_{e} Y_{e}^{\dagger}\right)-m_{l}^{2} Y_{e}^{\dagger} Y_{e} \operatorname{Tr}\left(Y_{e} Y_{e}^{\dagger}\right)-2 Y_{e}^{\dagger} m_{e}^{2} Y_{e} \operatorname{Tr}\left(Y_{e} Y_{e}^{\dagger}\right) \\
& -Y_{e}^{\dagger} Y_{e} m_{l}^{2} \operatorname{Tr}\left(Y_{e} Y_{e}^{\dagger}\right)-6 T_{e}^{\dagger} Y_{e} \operatorname{Tr}\left(Y_{d}^{\dagger} T_{d}\right)-2 T_{e}^{\dagger} Y_{e} \operatorname{Tr}\left(Y_{e}^{\dagger} T_{e}\right)
\end{aligned}
$$




$$
\begin{aligned}
& -6 Y_{e}^{\dagger} T_{e} \operatorname{Tr}\left(T_{d}^{*} Y_{d}^{T}\right)-6 Y_{e}^{\dagger} Y_{e} \operatorname{Tr}\left(T_{d}^{*} T_{d}^{T}\right)-2 Y_{e}^{\dagger} T_{e} \operatorname{Tr}\left(T_{e}^{*} Y_{e}^{T}\right) \\
& -2 Y_{e}^{\dagger} Y_{e} \operatorname{Tr}\left(T_{e}^{*} T_{e}^{T}\right)-6 Y_{e}^{\dagger} Y_{e} \operatorname{Tr}\left(m_{d}^{2} Y_{d} Y_{d}^{\dagger}\right)-2 Y_{e}^{\dagger} Y_{e} \operatorname{Tr}\left(m_{e}^{2} Y_{e} Y_{e}^{\dagger}\right) \\
& -2 Y_{e}^{\dagger} Y_{e} \operatorname{Tr}\left(m_{l}^{2} Y_{e}^{\dagger} Y_{e}\right)-6 Y_{e}^{\dagger} Y_{e} \operatorname{Tr}\left(m_{q}^{2} Y_{d}^{\dagger} Y_{d}\right) \\
& \beta_{m_{H_{d}}^{2}}^{(1)}=-\frac{6}{5} g_{1}^{2}\left|M_{1}\right|^{2}-6 g_{2}^{2}\left|M_{2}\right|^{2}-\sqrt{\frac{3}{5}} g_{1} \sigma_{1,1}+6 m_{H_{d}}^{2} \operatorname{Tr}\left(Y_{d} Y_{d}^{\dagger}\right)+2 m_{H_{d}}^{2} \operatorname{Tr}\left(Y_{e} Y_{e}^{\dagger}\right)+6 \operatorname{Tr}\left(T_{d}^{*} T_{d}^{T}\right) \\
& +2 \operatorname{Tr}\left(T_{e}^{*} T_{e}^{T}\right)+6 \operatorname{Tr}\left(m_{d}^{2} Y_{d} Y_{d}^{\dagger}\right)+2 \operatorname{Tr}\left(m_{e}^{2} Y_{e} Y_{e}^{\dagger}\right)+2 \operatorname{Tr}\left(m_{l}^{2} Y_{e}^{\dagger} Y_{e}\right)+6 \operatorname{Tr}\left(m_{q}^{2} Y_{d}^{\dagger} Y_{d}\right) \\
& \beta_{m_{H_{d}}^{2}}^{(2)}=\frac{1}{25}\left(15 g_{2}^{2}\left(3 g_{1}^{2}\left(2 M_{2}+M_{1}\right)+55 g_{2}^{2} M_{2}\right) M_{2}^{*}\right. \\
& +g_{1}^{2} M_{1}^{*}\left(729 g_{1}^{2} M_{1}+90 g_{2}^{2} M_{1}+45 g_{2}^{2} M_{2}-40 M_{1} \operatorname{Tr}\left(Y_{d} Y_{d}^{\dagger}\right)+120 M_{1} \operatorname{Tr}\left(Y_{e} Y_{e}^{\dagger}\right)+20 \operatorname{Tr}\left(Y_{d}^{\dagger} T_{d}\right)\right. \\
& \left.-60 \operatorname{Tr}\left(Y_{e}^{\dagger} T_{e}\right)\right) \\
& +10\left(15 g_{2}^{4} \sigma_{2,2}+3 g_{1}^{2} \sigma_{2,11}-2 \sqrt{15} g_{1} \sigma_{3,1}+\left(160 g_{3}^{2}\left|M_{3}\right|^{2}-2 g_{1}^{2} m_{H_{d}}^{2}+80 g_{3}^{2} m_{H_{d}}^{2}\right) \operatorname{Tr}\left(Y_{d} Y_{d}^{\dagger}\right)\right. \\
& +6 g_{1}^{2} m_{H_{d}}^{2} \operatorname{Tr}\left(Y_{e} Y_{e}^{\dagger}\right)-80 g_{3}^{2} M_{3}^{*} \operatorname{Tr}\left(Y_{d}^{\dagger} T_{d}\right)+2 g_{1}^{2} M_{1} \operatorname{Tr}\left(T_{d}^{*} Y_{d}^{T}\right)-80 g_{3}^{2} M_{3} \operatorname{Tr}\left(T_{d}^{*} Y_{d}^{T}\right) \\
& -2 g_{1}^{2} \operatorname{Tr}\left(T_{d}^{*} T_{d}^{T}\right)+80 g_{3}^{2} \operatorname{Tr}\left(T_{d}^{*} T_{d}^{T}\right)-6 g_{1}^{2} M_{1} \operatorname{Tr}\left(T_{e}^{*} Y_{e}^{T}\right)+6 g_{1}^{2} \operatorname{Tr}\left(T_{e}^{*} T_{e}^{T}\right) \\
& -2 g_{1}^{2} \operatorname{Tr}\left(m_{d}^{2} Y_{d} Y_{d}^{\dagger}\right)+80 g_{3}^{2} \operatorname{Tr}\left(m_{d}^{2} Y_{d} Y_{d}^{\dagger}\right)+6 g_{1}^{2} \operatorname{Tr}\left(m_{e}^{2} Y_{e} Y_{e}^{\dagger}\right)+6 g_{1}^{2} \operatorname{Tr}\left(m_{l}^{2} Y_{e}^{\dagger} Y_{e}\right) \\
& -2 g_{1}^{2} \operatorname{Tr}\left(m_{q}^{2} Y_{d}^{\dagger} Y_{d}\right)+80 g_{3}^{2} \operatorname{Tr}\left(m_{q}^{2} Y_{d}^{\dagger} Y_{d}\right)-90 m_{H_{d}}^{2} \operatorname{Tr}\left(Y_{d} Y_{d}^{\dagger} Y_{d} Y_{d}^{\dagger}\right)-90 \operatorname{Tr}\left(Y_{d} Y_{d}^{\dagger} T_{d} T_{d}^{\dagger}\right) \\
& -15 m_{H_{d}}^{2} \operatorname{Tr}\left(Y_{d} Y_{u}^{\dagger} Y_{u} Y_{d}^{\dagger}\right)-15 m_{H_{u}}^{2} \operatorname{Tr}\left(Y_{d} Y_{u}^{\dagger} Y_{u} Y_{d}^{\dagger}\right)-15 \operatorname{Tr}\left(Y_{d} Y_{u}^{\dagger} T_{u} T_{d}^{\dagger}\right) \\
& -90 \operatorname{Tr}\left(Y_{d} T_{d}^{\dagger} T_{d} Y_{d}^{\dagger}\right)-15 \operatorname{Tr}\left(Y_{d} T_{u}^{\dagger} T_{u} Y_{d}^{\dagger}\right)-30 m_{H_{d}}^{2} \operatorname{Tr}\left(Y_{e} Y_{e}^{\dagger} Y_{e} Y_{e}^{\dagger}\right)-30 \operatorname{Tr}\left(Y_{e} Y_{e}^{\dagger} T_{e} T_{e}^{\dagger}\right) \\
& -30 \operatorname{Tr}\left(Y_{e} T_{e}^{\dagger} T_{e} Y_{e}^{\dagger}\right)-15 \operatorname{Tr}\left(Y_{u} Y_{d}^{\dagger} T_{d} T_{u}^{\dagger}\right)-15 \operatorname{Tr}\left(Y_{u} T_{d}^{\dagger} T_{d} Y_{u}^{\dagger}\right)-90 \operatorname{Tr}\left(m_{d}^{2} Y_{d} Y_{d}^{\dagger} Y_{d} Y_{d}^{\dagger}\right) \\
& -15 \operatorname{Tr}\left(m_{d}^{2} Y_{d} Y_{u}^{\dagger} Y_{u} Y_{d}^{\dagger}\right)-30 \operatorname{Tr}\left(m_{e}^{2} Y_{e} Y_{e}^{\dagger} Y_{e} Y_{e}^{\dagger}\right)-30 \operatorname{Tr}\left(m_{l}^{2} Y_{e}^{\dagger} Y_{e} Y_{e}^{\dagger} Y_{e}\right)-90 \operatorname{Tr}\left(m_{q}^{2} Y_{d}^{\dagger} Y_{d} Y_{d}^{\dagger} Y_{d}\right) \\
& \left.\left.-15 \operatorname{Tr}\left(m_{q}^{2} Y_{d}^{\dagger} Y_{d} Y_{u}^{\dagger} Y_{u}\right)-15 \operatorname{Tr}\left(m_{q}^{2} Y_{u}^{\dagger} Y_{u} Y_{d}^{\dagger} Y_{d}\right)-15 \operatorname{Tr}\left(m_{u}^{2} Y_{u} Y_{d}^{\dagger} Y_{d} Y_{u}^{\dagger}\right)\right)\right) \\
& \beta_{m_{H_{u}}^{2}}^{(1)}=-\frac{6}{5} g_{1}^{2}\left|M_{1}\right|^{2}-6 g_{2}^{2}\left|M_{2}\right|^{2}+\sqrt{\frac{3}{5}} g_{1} \sigma_{1,1}+6 m_{H_{u}}^{2} \operatorname{Tr}\left(Y_{u} Y_{u}^{\dagger}\right)+6 \operatorname{Tr}\left(T_{u}^{*} T_{u}^{T}\right)+6 \operatorname{Tr}\left(m_{q}^{2} Y_{u}^{\dagger} Y_{u}\right) \\
& +6 \operatorname{Tr}\left(m_{u}^{2} Y_{u} Y_{u}^{\dagger}\right) \\
& \beta_{m_{H_{u}}^{2}}^{(2)}=+\frac{3}{5} g_{2}^{2}\left(3 g_{1}^{2}\left(2 M_{2}+M_{1}\right)+55 g_{2}^{2} M_{2}\right) M_{2}^{*}+6 g_{2}^{4} \sigma_{2,2}+\frac{6}{5} g_{1}^{2} \sigma_{2,11}+4 \sqrt{\frac{3}{5}} g_{1} \sigma_{3,1} \\
& +\frac{8}{5} g_{1}^{2} m_{H_{u}}^{2} \operatorname{Tr}\left(Y_{u} Y_{u}^{\dagger}\right)+32 g_{3}^{2} m_{H_{u}}^{2} \operatorname{Tr}\left(Y_{u} Y_{u}^{\dagger}\right)+64 g_{3}^{2}\left|M_{3}\right|^{2} \operatorname{Tr}\left(Y_{u} Y_{u}^{\dagger}\right) \\
& +\frac{1}{25} g_{1}^{2} M_{1}^{*}\left(-40 \operatorname{Tr}\left(Y_{u}^{\dagger} T_{u}\right)+45 g_{2}^{2} M_{2}+729 g_{1}^{2} M_{1}+80 M_{1} \operatorname{Tr}\left(Y_{u} Y_{u}^{\dagger}\right)+90 g_{2}^{2} M_{1}\right) \\
& -32 g_{3}^{2} M_{3}^{*} \operatorname{Tr}\left(Y_{u}^{\dagger} T_{u}\right)-\frac{8}{5} g_{1}^{2} M_{1} \operatorname{Tr}\left(T_{u}^{*} Y_{u}^{T}\right)-32 g_{3}^{2} M_{3} \operatorname{Tr}\left(T_{u}^{*} Y_{u}^{T}\right)+\frac{8}{5} g_{1}^{2} \operatorname{Tr}\left(T_{u}^{*} T_{u}^{T}\right) \\
& +32 g_{3}^{2} \operatorname{Tr}\left(T_{u}^{*} T_{u}^{T}\right)+\frac{8}{5} g_{1}^{2} \operatorname{Tr}\left(m_{q}^{2} Y_{u}^{\dagger} Y_{u}\right)+32 g_{3}^{2} \operatorname{Tr}\left(m_{q}^{2} Y_{u}^{\dagger} Y_{u}\right)+\frac{8}{5} g_{1}^{2} \operatorname{Tr}\left(m_{u}^{2} Y_{u} Y_{u}^{\dagger}\right) \\
& +32 g_{3}^{2} \operatorname{Tr}\left(m_{u}^{2} Y_{u} Y_{u}^{\dagger}\right)-6 m_{H_{d}}^{2} \operatorname{Tr}\left(Y_{d} Y_{u}^{\dagger} Y_{u} Y_{d}^{\dagger}\right)-6 m_{H_{u}}^{2} \operatorname{Tr}\left(Y_{d} Y_{u}^{\dagger} Y_{u} Y_{d}^{\dagger}\right)
\end{aligned}
$$




$$
\begin{aligned}
& -6 \operatorname{Tr}\left(Y_{d} Y_{u}^{\dagger} T_{u} T_{d}^{\dagger}\right)-6 \operatorname{Tr}\left(Y_{d} T_{u}^{\dagger} T_{u} Y_{d}^{\dagger}\right)-6 \operatorname{Tr}\left(Y_{u} Y_{d}^{\dagger} T_{d} T_{u}^{\dagger}\right)-36 m_{H_{u}}^{2} \operatorname{Tr}\left(Y_{u} Y_{u}^{\dagger} Y_{u} Y_{u}^{\dagger}\right) \\
& -36 \operatorname{Tr}\left(Y_{u} Y_{u}^{\dagger} T_{u} T_{u}^{\dagger}\right)-6 \operatorname{Tr}\left(Y_{u} T_{d}^{\dagger} T_{d} Y_{u}^{\dagger}\right)-36 \operatorname{Tr}\left(Y_{u} T_{u}^{\dagger} T_{u} Y_{u}^{\dagger}\right) \\
& -6 \operatorname{Tr}\left(m_{d}^{2} Y_{d} Y_{u}^{\dagger} Y_{u} Y_{d}^{\dagger}\right)-6 \operatorname{Tr}\left(m_{q}^{2} Y_{d}^{\dagger} Y_{d} Y_{u}^{\dagger} Y_{u}\right)-6 \operatorname{Tr}\left(m_{q}^{2} Y_{u}^{\dagger} Y_{u} Y_{d}^{\dagger} Y_{d}\right) \\
& -36 \operatorname{Tr}\left(m_{q}^{2} Y_{u}^{\dagger} Y_{u} Y_{u}^{\dagger} Y_{u}\right)-6 \operatorname{Tr}\left(m_{u}^{2} Y_{u} Y_{d}^{\dagger} Y_{d} Y_{u}^{\dagger}\right)-36 \operatorname{Tr}\left(m_{u}^{2} Y_{u} Y_{u}^{\dagger} Y_{u} Y_{u}^{\dagger}\right) \\
& \beta_{m_{d}^{2}}^{(1)}=-\frac{8}{15} g_{1}^{2} \mathbf{1}\left|M_{1}\right|^{2}-\frac{32}{3} g_{3}^{2} \mathbf{1}\left|M_{3}\right|^{2}+4 m_{H_{d}}^{2} Y_{d} Y_{d}^{\dagger}+4 T_{d} T_{d}^{\dagger}+2 m_{d}^{2} Y_{d} Y_{d}^{\dagger}+4 Y_{d} m_{q}^{2} Y_{d}^{\dagger} \\
& +2 Y_{d} Y_{d}^{\dagger} m_{d}^{2}+2 \frac{1}{\sqrt{15}} g_{1} 1 \sigma_{1,1} \\
& \beta_{m_{d}^{2}}^{(2)}=+\frac{64}{45} g_{3}^{2}\left(-30 g_{3}^{2} M_{3}+g_{1}^{2}\left(2 M_{3}+M_{1}\right)\right) \mathbf{1} M_{3}^{*}+\frac{4}{5} g_{1}^{2} m_{H_{d}}^{2} Y_{d} Y_{d}^{\dagger}+12 g_{2}^{2} m_{H_{d}}^{2} Y_{d} Y_{d}^{\dagger} \\
& +24 g_{2}^{2}\left|M_{2}\right|^{2} Y_{d} Y_{d}^{\dagger}-\frac{4}{5} g_{1}^{2} M_{1} Y_{d} T_{d}^{\dagger}-12 g_{2}^{2} M_{2} Y_{d} T_{d}^{\dagger} \\
& +\frac{4}{225} g_{1}^{2} M_{1}^{*}\left(2\left(357 g_{1}^{2} M_{1}+40 g_{3}^{2}\left(2 M_{1}+M_{3}\right)\right) 1-45 T_{d} Y_{d}^{\dagger}+90 M_{1} Y_{d} Y_{d}^{\dagger}\right)-12 g_{2}^{2} M_{2}^{*} T_{d} Y_{d}^{\dagger} \\
& +\frac{4}{5} g_{1}^{2} T_{d} T_{d}^{\dagger}+12 g_{2}^{2} T_{d} T_{d}^{\dagger}+\frac{2}{5} g_{1}^{2} m_{d}^{2} Y_{d} Y_{d}^{\dagger}+6 g_{2}^{2} m_{d}^{2} Y_{d} Y_{d}^{\dagger} \\
& +\frac{4}{5} g_{1}^{2} Y_{d} m_{q}^{2} Y_{d}^{\dagger}+12 g_{2}^{2} Y_{d} m_{q}^{2} Y_{d}^{\dagger}+\frac{2}{5} g_{1}^{2} Y_{d} Y_{d}^{\dagger} m_{d}^{2}+6 g_{2}^{2} Y_{d} Y_{d}^{\dagger} m_{d}^{2} \\
& -8 m_{H_{d}}^{2} Y_{d} Y_{d}^{\dagger} Y_{d} Y_{d}^{\dagger}-4 Y_{d} Y_{d}^{\dagger} T_{d} T_{d}^{\dagger}-4 m_{H_{d}}^{2} Y_{d} Y_{u}^{\dagger} Y_{u} Y_{d}^{\dagger} \\
& -4 m_{H_{u}}^{2} Y_{d} Y_{u}^{\dagger} Y_{u} Y_{d}^{\dagger}-4 Y_{d} Y_{u}^{\dagger} T_{u} T_{d}^{\dagger}-4 Y_{d} T_{d}^{\dagger} T_{d} Y_{d}^{\dagger}-4 Y_{d} T_{u}^{\dagger} T_{u} Y_{d}^{\dagger} \\
& -4 T_{d} Y_{d}^{\dagger} Y_{d} T_{d}^{\dagger}-4 T_{d} Y_{u}^{\dagger} Y_{u} T_{d}^{\dagger}-4 T_{d} T_{d}^{\dagger} Y_{d} Y_{d}^{\dagger}-4 T_{d} T_{u}^{\dagger} Y_{u} Y_{d}^{\dagger} \\
& -2 m_{d}^{2} Y_{d} Y_{d}^{\dagger} Y_{d} Y_{d}^{\dagger}-2 m_{d}^{2} Y_{d} Y_{u}^{\dagger} Y_{u} Y_{d}^{\dagger}-4 Y_{d} m_{q}^{2} Y_{d}^{\dagger} Y_{d} Y_{d}^{\dagger}-4 Y_{d} m_{q}^{2} Y_{u}^{\dagger} Y_{u} Y_{d}^{\dagger} \\
& -4 Y_{d} Y_{d}^{\dagger} m_{d}^{2} Y_{d} Y_{d}^{\dagger}-4 Y_{d} Y_{d}^{\dagger} Y_{d} m_{q}^{2} Y_{d}^{\dagger}-2 Y_{d} Y_{d}^{\dagger} Y_{d} Y_{d}^{\dagger} m_{d}^{2}-4 Y_{d} Y_{u}^{\dagger} m_{u}^{2} Y_{u} Y_{d}^{\dagger} \\
& -4 Y_{d} Y_{u}^{\dagger} Y_{u} m_{q}^{2} Y_{d}^{\dagger}-2 Y_{d} Y_{u}^{\dagger} Y_{u} Y_{d}^{\dagger} m_{d}^{2}+\frac{32}{3} g_{3}^{4} \mathbf{1} \sigma_{2,3}+\frac{8}{15} g_{1}^{2} \mathbf{1} \sigma_{2,11}+8 \frac{1}{\sqrt{15}} g_{1} \mathbf{1} \sigma_{3,1} \\
& -24 m_{H_{d}}^{2} Y_{d} Y_{d}^{\dagger} \operatorname{Tr}\left(Y_{d} Y_{d}^{\dagger}\right)-12 T_{d} T_{d}^{\dagger} \operatorname{Tr}\left(Y_{d} Y_{d}^{\dagger}\right)-6 m_{d}^{2} Y_{d} Y_{d}^{\dagger} \operatorname{Tr}\left(Y_{d} Y_{d}^{\dagger}\right) \\
& -12 Y_{d} m_{q}^{2} Y_{d}^{\dagger} \operatorname{Tr}\left(Y_{d} Y_{d}^{\dagger}\right)-6 Y_{d} Y_{d}^{\dagger} m_{d}^{2} \operatorname{Tr}\left(Y_{d} Y_{d}^{\dagger}\right)-8 m_{H_{d}}^{2} Y_{d} Y_{d}^{\dagger} \operatorname{Tr}\left(Y_{e} Y_{e}^{\dagger}\right) \\
& -4 T_{d} T_{d}^{\dagger} \operatorname{Tr}\left(Y_{e} Y_{e}^{\dagger}\right)-2 m_{d}^{2} Y_{d} Y_{d}^{\dagger} \operatorname{Tr}\left(Y_{e} Y_{e}^{\dagger}\right)-4 Y_{d} m_{q}^{2} Y_{d}^{\dagger} \operatorname{Tr}\left(Y_{e} Y_{e}^{\dagger}\right) \\
& -2 Y_{d} Y_{d}^{\dagger} m_{d}^{2} \operatorname{Tr}\left(Y_{e} Y_{e}^{\dagger}\right)-12 Y_{d} T_{d}^{\dagger} \operatorname{Tr}\left(Y_{d}^{\dagger} T_{d}\right)-4 Y_{d} T_{d}^{\dagger} \operatorname{Tr}\left(Y_{e}^{\dagger} T_{e}\right) \\
& -12 T_{d} Y_{d}^{\dagger} \operatorname{Tr}\left(T_{d}^{*} Y_{d}^{T}\right)-12 Y_{d} Y_{d}^{\dagger} \operatorname{Tr}\left(T_{d}^{*} T_{d}^{T}\right)-4 T_{d} Y_{d}^{\dagger} \operatorname{Tr}\left(T_{e}^{*} Y_{e}^{T}\right) \\
& -4 Y_{d} Y_{d}^{\dagger} \operatorname{Tr}\left(T_{e}^{*} T_{e}^{T}\right)-12 Y_{d} Y_{d}^{\dagger} \operatorname{Tr}\left(m_{d}^{2} Y_{d} Y_{d}^{\dagger}\right)-4 Y_{d} Y_{d}^{\dagger} \operatorname{Tr}\left(m_{e}^{2} Y_{e} Y_{e}^{\dagger}\right) \\
& -4 Y_{d} Y_{d}^{\dagger} \operatorname{Tr}\left(m_{l}^{2} Y_{e}^{\dagger} Y_{e}\right)-12 Y_{d} Y_{d}^{\dagger} \operatorname{Tr}\left(m_{q}^{2} Y_{d}^{\dagger} Y_{d}\right) \\
& \beta_{m_{u}^{2}}^{(1)}=-\frac{32}{15} g_{1}^{2} \mathbf{1}\left|M_{1}\right|^{2}-\frac{32}{3} g_{3}^{2} \mathbf{1}\left|M_{3}\right|^{2}+4 m_{H_{u}}^{2} Y_{u} Y_{u}^{\dagger}+4 T_{u} T_{u}^{\dagger}+2 m_{u}^{2} Y_{u} Y_{u}^{\dagger}+4 Y_{u} m_{q}^{2} Y_{u}^{\dagger} \\
& +2 Y_{u} Y_{u}^{\dagger} m_{u}^{2}-4 \frac{1}{\sqrt{15}} g_{1} \mathbf{1} \sigma_{1,1}
\end{aligned}
$$




$$
\begin{aligned}
& \beta_{m_{u}^{2}}^{(2)}=-\frac{128}{45} g_{3}^{2}\left(15 g_{3}^{2} M_{3}-2 g_{1}^{2}\left(2 M_{3}+M_{1}\right)\right) \mathbf{1} M_{3}^{*}-\frac{4}{5} g_{1}^{2} m_{H_{u}}^{2} Y_{u} Y_{u}^{\dagger}+12 g_{2}^{2} m_{H_{u}}^{2} Y_{u} Y_{u}^{\dagger} \\
& +24 g_{2}^{2}\left|M_{2}\right|^{2} Y_{u} Y_{u}^{\dagger}+\frac{4}{5} g_{1}^{2} M_{1} Y_{u} T_{u}^{\dagger}-12 g_{2}^{2} M_{2} Y_{u} T_{u}^{\dagger}-12 g_{2}^{2} M_{2}^{*} T_{u} Y_{u}^{\dagger} \\
& +\frac{4}{45} g_{1}^{2} M_{1}^{*}\left(8\left(75 g_{1}^{2} M_{1}+8 g_{3}^{2}\left(2 M_{1}+M_{3}\right)\right) \mathbf{1}+9\left(-2 M_{1} Y_{u} Y_{u}^{\dagger}+T_{u} Y_{u}^{\dagger}\right)\right)-\frac{4}{5} g_{1}^{2} T_{u} T_{u}^{\dagger} \\
& +12 g_{2}^{2} T_{u} T_{u}^{\dagger}-\frac{2}{5} g_{1}^{2} m_{u}^{2} Y_{u} Y_{u}^{\dagger}+6 g_{2}^{2} m_{u}^{2} Y_{u} Y_{u}^{\dagger}-\frac{4}{5} g_{1}^{2} Y_{u} m_{q}^{2} Y_{u}^{\dagger} \\
& +12 g_{2}^{2} Y_{u} m_{q}^{2} Y_{u}^{\dagger}-\frac{2}{5} g_{1}^{2} Y_{u} Y_{u}^{\dagger} m_{u}^{2}+6 g_{2}^{2} Y_{u} Y_{u}^{\dagger} m_{u}^{2}-4 m_{H_{d}}^{2} Y_{u} Y_{d}^{\dagger} Y_{d} Y_{u}^{\dagger} \\
& -4 m_{H_{u}}^{2} Y_{u} Y_{d}^{\dagger} Y_{d} Y_{u}^{\dagger}-4 Y_{u} Y_{d}^{\dagger} T_{d} T_{u}^{\dagger}-8 m_{H_{u}}^{2} Y_{u} Y_{u}^{\dagger} Y_{u} Y_{u}^{\dagger}-4 Y_{u} Y_{u}^{\dagger} T_{u} T_{u}^{\dagger} \\
& -4 Y_{u} T_{d}^{\dagger} T_{d} Y_{u}^{\dagger}-4 Y_{u} T_{u}^{\dagger} T_{u} Y_{u}^{\dagger}-4 T_{u} Y_{d}^{\dagger} Y_{d} T_{u}^{\dagger}-4 T_{u} Y_{u}^{\dagger} Y_{u} T_{u}^{\dagger} \\
& -4 T_{u} T_{d}^{\dagger} Y_{d} Y_{u}^{\dagger}-4 T_{u} T_{u}^{\dagger} Y_{u} Y_{u}^{\dagger}-2 m_{u}^{2} Y_{u} Y_{d}^{\dagger} Y_{d} Y_{u}^{\dagger}-2 m_{u}^{2} Y_{u} Y_{u}^{\dagger} Y_{u} Y_{u}^{\dagger} \\
& -4 Y_{u} m_{q}^{2} Y_{d}^{\dagger} Y_{d} Y_{u}^{\dagger}-4 Y_{u} m_{q}^{2} Y_{u}^{\dagger} Y_{u} Y_{u}^{\dagger}-4 Y_{u} Y_{d}^{\dagger} m_{d}^{2} Y_{d} Y_{u}^{\dagger} \\
& -4 Y_{u} Y_{d}^{\dagger} Y_{d} m_{q}^{2} Y_{u}^{\dagger}-2 Y_{u} Y_{d}^{\dagger} Y_{d} Y_{u}^{\dagger} m_{u}^{2}-4 Y_{u} Y_{u}^{\dagger} m_{u}^{2} Y_{u} Y_{u}^{\dagger}-4 Y_{u} Y_{u}^{\dagger} Y_{u} m_{q}^{2} Y_{u}^{\dagger} \\
& -2 Y_{u} Y_{u}^{\dagger} Y_{u} Y_{u}^{\dagger} m_{u}^{2}+\frac{32}{3} g_{3}^{4} \mathbf{1} \sigma_{2,3}+\frac{32}{15} g_{1}^{2} \mathbf{1} \sigma_{2,11}-16 \frac{1}{\sqrt{15}} g_{1} \mathbf{1} \sigma_{3,1}-24 m_{H_{u}}^{2} Y_{u} Y_{u}^{\dagger} \operatorname{Tr}\left(Y_{u} Y_{u}^{\dagger}\right) \\
& -12 T_{u} T_{u}^{\dagger} \operatorname{Tr}\left(Y_{u} Y_{u}^{\dagger}\right)-6 m_{u}^{2} Y_{u} Y_{u}^{\dagger} \operatorname{Tr}\left(Y_{u} Y_{u}^{\dagger}\right)-12 Y_{u} m_{q}^{2} Y_{u}^{\dagger} \operatorname{Tr}\left(Y_{u} Y_{u}^{\dagger}\right) \\
& -6 Y_{u} Y_{u}^{\dagger} m_{u}^{2} \operatorname{Tr}\left(Y_{u} Y_{u}^{\dagger}\right)-12 Y_{u} T_{u}^{\dagger} \operatorname{Tr}\left(Y_{u}^{\dagger} T_{u}\right)-12 T_{u} Y_{u}^{\dagger} \operatorname{Tr}\left(T_{u}^{*} Y_{u}^{T}\right) \\
& -12 Y_{u} Y_{u}^{\dagger} \operatorname{Tr}\left(T_{u}^{*} T_{u}^{T}\right)-12 Y_{u} Y_{u}^{\dagger} \operatorname{Tr}\left(m_{q}^{2} Y_{u}^{\dagger} Y_{u}\right)-12 Y_{u} Y_{u}^{\dagger} \operatorname{Tr}\left(m_{u}^{2} Y_{u} Y_{u}^{\dagger}\right) \\
& \beta_{m_{e}^{2}}^{(1)}=-\frac{24}{5} g_{1}^{2} \mathbf{1}\left|M_{1}\right|^{2}+2\left(2 m_{H_{d}}^{2} Y_{e} Y_{e}^{\dagger}+2 T_{e} T_{e}^{\dagger}+2 Y_{e} m_{l}^{2} Y_{e}^{\dagger}+m_{e}^{2} Y_{e} Y_{e}^{\dagger}+Y_{e} Y_{e}^{\dagger} m_{e}^{2}\right) \\
& +2 \sqrt{\frac{3}{5}} g_{1} \mathbf{1} \sigma_{1,1} \\
& \beta_{m_{e}^{2}}^{(2)}=\frac{2}{5}\left(-6 g_{1}^{2} m_{H_{d}}^{2} Y_{e} Y_{e}^{\dagger}+30 g_{2}^{2} m_{H_{d}}^{2} Y_{e} Y_{e}^{\dagger}+60 g_{2}^{2}\left|M_{2}\right|^{2} Y_{e} Y_{e}^{\dagger}+6 g_{1}^{2} M_{1} Y_{e} T_{e}^{\dagger}\right. \\
& -30 g_{2}^{2} M_{2} Y_{e} T_{e}^{\dagger}-30 g_{2}^{2} M_{2}^{*} T_{e} Y_{e}^{\dagger}+6 g_{1}^{2} M_{1}^{*}\left(-2 M_{1} Y_{e} Y_{e}^{\dagger}+54 g_{1}^{2} M_{1} \mathbf{1}+T_{e} Y_{e}^{\dagger}\right) \\
& -6 g_{1}^{2} T_{e} T_{e}^{\dagger}+30 g_{2}^{2} T_{e} T_{e}^{\dagger}-3 g_{1}^{2} m_{e}^{2} Y_{e} Y_{e}^{\dagger}+15 g_{2}^{2} m_{e}^{2} Y_{e} Y_{e}^{\dagger} \\
& -6 g_{1}^{2} Y_{e} m_{l}^{2} Y_{e}^{\dagger}+30 g_{2}^{2} Y_{e} m_{l}^{2} Y_{e}^{\dagger}-3 g_{1}^{2} Y_{e} Y_{e}^{\dagger} m_{e}^{2}+15 g_{2}^{2} Y_{e} Y_{e}^{\dagger} m_{e}^{2} \\
& -20 m_{H_{d}}^{2} Y_{e} Y_{e}^{\dagger} Y_{e} Y_{e}^{\dagger}-10 Y_{e} Y_{e}^{\dagger} T_{e} T_{e}^{\dagger}-10 Y_{e} T_{e}^{\dagger} T_{e} Y_{e}^{\dagger}-10 T_{e} Y_{e}^{\dagger} Y_{e} T_{e}^{\dagger} \\
& -10 T_{e} T_{e}^{\dagger} Y_{e} Y_{e}^{\dagger}-5 m_{e}^{2} Y_{e} Y_{e}^{\dagger} Y_{e} Y_{e}^{\dagger}-10 Y_{e} m_{l}^{2} Y_{e}^{\dagger} Y_{e} Y_{e}^{\dagger}-10 Y_{e} Y_{e}^{\dagger} m_{e}^{2} Y_{e} Y_{e}^{\dagger} \\
& -10 Y_{e} Y_{e}^{\dagger} Y_{e} m_{l}^{2} Y_{e}^{\dagger}-5 Y_{e} Y_{e}^{\dagger} Y_{e} Y_{e}^{\dagger} m_{e}^{2}+4 g_{1} 1\left(3 g_{1} \sigma_{2,11}+\sqrt{15} \sigma_{3,1}\right) \\
& -60 m_{H_{d}}^{2} Y_{e} Y_{e}^{\dagger} \operatorname{Tr}\left(Y_{d} Y_{d}^{\dagger}\right)-30 T_{e} T_{e}^{\dagger} \operatorname{Tr}\left(Y_{d} Y_{d}^{\dagger}\right)-15 m_{e}^{2} Y_{e} Y_{e}^{\dagger} \operatorname{Tr}\left(Y_{d} Y_{d}^{\dagger}\right) \\
& -30 Y_{e} m_{l}^{2} Y_{e}^{\dagger} \operatorname{Tr}\left(Y_{d} Y_{d}^{\dagger}\right)-15 Y_{e} Y_{e}^{\dagger} m_{e}^{2} \operatorname{Tr}\left(Y_{d} Y_{d}^{\dagger}\right)-20 m_{H_{d}}^{2} Y_{e} Y_{e}^{\dagger} \operatorname{Tr}\left(Y_{e} Y_{e}^{\dagger}\right) \\
& -10 T_{e} T_{e}^{\dagger} \operatorname{Tr}\left(Y_{e} Y_{e}^{\dagger}\right)-5 m_{e}^{2} Y_{e} Y_{e}^{\dagger} \operatorname{Tr}\left(Y_{e} Y_{e}^{\dagger}\right)-10 Y_{e} m_{l}^{2} Y_{e}^{\dagger} \operatorname{Tr}\left(Y_{e} Y_{e}^{\dagger}\right) \\
& -5 Y_{e} Y_{e}^{\dagger} m_{e}^{2} \operatorname{Tr}\left(Y_{e} Y_{e}^{\dagger}\right)-30 Y_{e} T_{e}^{\dagger} \operatorname{Tr}\left(Y_{d}^{\dagger} T_{d}\right)-10 Y_{e} T_{e}^{\dagger} \operatorname{Tr}\left(Y_{e}^{\dagger} T_{e}\right)
\end{aligned}
$$




$$
\begin{aligned}
&-30 T_{e} Y_{e}^{\dagger} \operatorname{Tr}\left(T_{d}^{*} Y_{d}^{T}\right)-30 Y_{e} Y_{e}^{\dagger} \operatorname{Tr}\left(T_{d}^{*} T_{d}^{T}\right)-10 T_{e} Y_{e}^{\dagger} \operatorname{Tr}\left(T_{e}^{*} Y_{e}^{T}\right) \\
&-10 Y_{e} Y_{e}^{\dagger} \operatorname{Tr}\left(T_{e}^{*} T_{e}^{T}\right)-30 Y_{e} Y_{e}^{\dagger} \operatorname{Tr}\left(m_{d}^{2} Y_{d} Y_{d}^{\dagger}\right)-10 Y_{e} Y_{e}^{\dagger} \operatorname{Tr}\left(m_{e}^{2} Y_{e} Y_{e}^{\dagger}\right) \\
&\left.-10 Y_{e} Y_{e}^{\dagger} \operatorname{Tr}\left(m_{l}^{2} Y_{e}^{\dagger} Y_{e}\right)-30 Y_{e} Y_{e}^{\dagger} \operatorname{Tr}\left(m_{q}^{2} Y_{d}^{\dagger} Y_{d}\right)\right) \\
& \beta_{m_{F_{+}}^{2}}^{(1)}= \frac{2}{5} g_{1}\left(-12 g_{1}\left|M_{1}\right|^{2}+\sqrt{15} \sigma_{1,1}\right) \\
& \beta_{m_{F_{+}}^{2}}^{(2)}=\frac{8}{5} g_{1}\left(3 g_{1} \sigma_{2,11}+81 g_{1}^{3}\left|M_{1}\right|^{2}+\sqrt{15} \sigma_{3,1}\right) \\
& \beta_{m_{F_{-}}^{2}=}^{(1)}=\frac{2}{5} g_{1}\left(12 g_{1}\left|M_{1}\right|^{2}+\sqrt{15} \sigma_{1,1}\right) \\
& \beta_{m_{F_{-}}^{2}}^{(2)}=\frac{8}{5} g_{1}\left(3 g_{1} \sigma_{2,11}+81 g_{1}^{3}\left|M_{1}\right|^{2}-\sqrt{15} \sigma_{3,1}\right)
\end{aligned}
$$

\section{B Renormalisation group equations for the 5D-SSM+F $\boldsymbol{F}^{ \pm}$}

In this appendix we supply the one-loop beta functions used in the main paper for the five dimensional model 1, model 2 and model 3, including the five dimensional Kaluza-Klein states and extra fields. Note that the RGEs for model 3 can be read off from model 1 and 2 as in every model the RGEs for fields in the bulk is similar to model 2 RGEs, and the RGEs for fields on the brane is similar to RGEs of model 1. The Higgs sector RGEs for model 3 are always in the bulk in both model 1 and 2 . We define $t=\log _{10} Q$ and $\beta_{A}=16 \pi^{2} d A / d t$. It is useful to also define the power law contribution, which may be written equivalently as

$$
(Q R)^{d}=10^{t} R
$$

\section{B.1 Gauge couplings}

The one-loop beta function for the gauge couplings if $t>\ln (1 / R) / \ln (10)$ are given by

$$
16 \pi^{2} \frac{d g_{i}(t)}{d t}=b_{\mathrm{MSSM}}^{i} g_{i}^{3}(t)+b_{5 D}^{i} g_{i}^{3}(t)(S(t)-1),
$$

where $i=1,2,3$ and $S(t)=R 10^{t}$, the power law contribution. For the $4 D S S M+F^{ \pm}$, $b^{i}=(39 / 5,1,-3)$ and for five dimensions $b_{5 D}^{i}=(18 / 5,-2,-6)+4 \eta$, where $\eta$ is the number of fermion generation in the bulk. The fine structure constants may be defined from $\alpha_{i}=g_{i}^{2} / 4 \pi$.

\section{B.2 Yukawa couplings}

The beta functions for the Yukawa couplings may be related to the matrices of anomalous dimensions

$$
\beta_{Y}^{i j k}=\gamma_{n}^{i} Y^{n j k}+\gamma_{n}^{i} Y^{i n k}+\gamma_{n}^{k} Y^{i j n} .
$$


B.2.1 Anomalous dimensions for model 1

$$
\begin{aligned}
\gamma_{\tilde{H}_{u}} & =3 \operatorname{Tr}\left(Y_{u} Y_{u}^{\dagger}\right)-\left(\frac{3}{10} g_{1}^{2}+\frac{3}{2} g_{2}^{2}\right) S(t) \\
\gamma_{\tilde{H}_{d}} & =3 \operatorname{Tr}\left(Y_{d} Y_{d}^{\dagger}\right)+\operatorname{Tr}\left(Y_{e} Y_{e}^{\dagger}\right)-\left(\frac{3}{10} g_{1}^{2}+\frac{3}{2} g_{2}^{2}\right) S(t) \\
\gamma_{\tilde{F}^{ \pm}} & =Y_{F} Y_{F}^{\dagger}-\frac{12}{10} g_{1}^{2} S(t) \\
\gamma_{\tilde{q}} & =\left(2\left(Y_{u} Y_{u}^{\dagger}+Y_{d} Y_{d}^{\dagger}\right)-\left(\frac{1}{15} g_{1}^{2}+3 g_{2}^{2}+\frac{16}{3} g_{3}^{2}\right)\right) S(t) \\
\gamma_{\tilde{u}} & =\left(4 Y_{u} Y_{u}^{\dagger}-\left(\frac{16}{15} g_{1}^{2}+\frac{16}{3} g_{3}^{2}\right)\right) S(t) \\
\gamma_{\tilde{d}} & =\left(4 Y_{d} Y_{d}^{\dagger}-\left(\frac{4}{15} g_{1}^{2}+\frac{16}{3} g_{3}^{2}\right)\right) S(t) \\
\gamma_{\tilde{l}} & =\left(2 Y_{e} Y_{e}^{\dagger}-\left(\frac{3}{5} g_{1}^{2}+3 g_{2}^{2}\right)\right) S(t) \\
\gamma_{\tilde{e}} & =\left(4 Y_{e} Y_{e}^{\dagger}-\frac{12}{5} g_{1}^{2}\right) S(t) .
\end{aligned}
$$

\section{B.2.2 Anomalous dimensions for model 2}

$$
\begin{aligned}
\gamma_{\tilde{H}_{u}} & =3 \operatorname{Tr}\left(Y_{u} Y_{u}^{\dagger}\right) \pi S(t)^{2}-\left(\frac{3}{10} g_{1}^{2}+\frac{3}{2} g_{2}^{2}\right) S(t) \\
\gamma_{\tilde{H}_{d}} & =\left(3 \operatorname{Tr}\left(Y_{d} Y_{d}^{\dagger}\right)+\operatorname{Tr}\left(Y_{e} Y_{e}^{\dagger}\right)\right) \pi S(t)^{2}-\left(\frac{3}{10} g_{1}^{2}+\frac{3}{2} g_{2}^{2}\right) S(t) \\
\gamma_{\tilde{F}^{ \pm}} & =Y_{F} Y_{F}^{\dagger} \pi S(t)^{2}-\frac{12}{10} g_{1}^{2} S(t) \\
\gamma_{\tilde{q}^{3}} & =\left(Y_{t} Y_{t}^{\dagger}+Y_{b} Y_{b}^{\dagger}\right) \pi S(t)^{2}-\left(\frac{1}{30} g_{1}^{2}+\frac{3}{2} g_{2}^{2}+\frac{8}{3} g_{3}^{2}\right) S(t) \\
\gamma_{\tilde{u}^{3}} & =2 Y_{t} Y_{t}^{\dagger} \pi S(t)^{2}-\left(\frac{8}{15} g_{1}^{2}+\frac{8}{3} g_{3}^{2}\right) S(t) \\
\gamma_{\tilde{d}^{3}} & =2 Y_{b} Y_{b}^{\dagger} \pi S(t)^{2}-\left(\frac{2}{15} g_{1}^{2}+\frac{8}{3} g_{3}^{2}\right) S(t) \\
\gamma_{\tilde{l}^{3}} & =Y_{\tau} Y_{\tau}^{\dagger} \pi S(t)^{2}-\left(\frac{3}{10} g_{1}^{2}+\frac{3}{2} g_{2}^{2}\right) S(t) \\
\gamma_{\tilde{e}^{3}} & =2 Y_{\tau} Y_{\tau}^{\dagger} \pi S(t)^{2}-\frac{6}{5} g_{1}^{2} S(t) .
\end{aligned}
$$




\section{B.2.3 Anomalous dimensions for model 3}

$$
\begin{aligned}
\gamma_{\tilde{H}_{u}} & =3 \operatorname{Tr}\left(Y_{t} Y_{t}^{\dagger}\right) \pi S(t)^{2}-\left(\frac{3}{10} g_{1}^{2}+\frac{3}{2} g_{2}^{2}\right) S(t) \\
\gamma_{\tilde{H}_{d}} & =\left(3 \operatorname{Tr}\left(Y_{b} Y_{b}^{\dagger}\right)+\operatorname{Tr}\left(Y_{\tau} Y_{\tau}^{\dagger}\right)\right) \pi S(t)^{2}-\left(\frac{3}{10} g_{1}^{2}+\frac{3}{2} g_{2}^{2}\right) S(t) \\
\gamma_{\tilde{F}^{ \pm}} & =Y_{F} Y_{F}^{\dagger} \pi S(t)^{2}-\frac{12}{10} g_{1}^{2} S(t) \\
\gamma_{\tilde{q}^{3}} & =2\left(Y_{t} Y_{t}^{\dagger}+Y_{b} Y_{b}^{\dagger}\right) S(t)^{2}-\left(\frac{1}{15} g_{1}^{2}+3 g_{2}^{2}+\frac{16}{3} g_{3}^{2}\right) S(t) \\
\gamma_{\tilde{u}^{3}} & =4 Y_{t} Y_{t}^{\dagger} S(t)-\left(\frac{16}{15} g_{1}^{2}+\frac{16}{3} g_{3}^{2}\right) S(t) \\
\gamma_{\tilde{d}^{3}} & =4 Y_{b} Y_{b}^{\dagger} S(t)-\left(\frac{4}{15} g_{1}^{2}+\frac{16}{3} g_{3}^{2}\right) S(t) \\
\gamma_{\tilde{l}^{3}} & =2 Y_{\tau} Y_{\tau}^{\dagger} S(t)-\left(\frac{3}{5} g_{1}^{2}+3 g_{2}^{2}\right) S(t) \\
\gamma_{\tilde{e}^{3}} & =4 Y_{\tau} Y_{\tau}^{\dagger} S(t)-\frac{12}{5} g_{1}^{2} S(t) .
\end{aligned}
$$

\section{B.2.4 Yukawa coupling RGEs for model 1}

The five dimensional contributions for model 1 are given by

$$
\begin{aligned}
& \beta_{(5 D) Y_{u}}^{(1)}=Y_{u}\left(\left(6 Y_{u}^{\dagger} Y_{u}+2 Y_{d}^{\dagger} Y_{d}+2 Y_{F}^{\dagger} Y_{F}\right)-\left(\frac{79}{30} g_{1}^{2}+\frac{9}{2} g_{2}^{2}+\frac{32}{3} g_{3}^{2}\right)\right) S(t) \\
& \beta_{(5 D) Y_{d}}^{(1)}=Y_{d}\left(\left(6 Y_{d}^{\dagger} Y_{d}+2 Y_{u}^{\dagger} Y_{u}+2 Y_{F}^{\dagger} Y_{F}\right)-\left(\frac{55}{30} g_{1}^{2}+\frac{9}{2} g_{2}^{2}+\frac{32}{3} g_{3}^{2}\right)\right) S(t) \\
& \beta_{(5 D) Y_{e}}^{(1)}=Y_{e}\left(6 Y_{e}^{\dagger} Y_{e}+2 Y_{F}^{\dagger} Y_{F}-\left(\frac{9}{2} g_{1}^{2}+\frac{9}{2} g_{2}^{2}\right)\right) S(t) \\
& \beta_{(5 D) Y_{F}}^{(1)}=Y_{F}\left(4 Y_{F}^{\dagger} Y_{F}-\left(3 g_{1}^{2}+3 g_{2}^{2}\right)\right) S(t) .
\end{aligned}
$$

\section{B.2.5 Yukawa coupling RGEs for model 2}

The five dimensional contributions for model 2 are given by

$$
\begin{aligned}
\beta_{(5 D) Y_{t}}^{(1)}= & Y_{t}\left(3 \operatorname{Tr}\left(Y_{t}^{\dagger} Y_{t}\right)+3 Y_{t}^{\dagger} Y_{t}+Y_{b}^{\dagger} Y_{b}+Y_{F}^{\dagger} Y_{F}\right) \pi S(t)^{2} \\
& -Y_{t}\left(\frac{31}{15} g_{1}^{2}+3 g_{2}^{2}+\frac{16}{3} g_{3}^{2}\right) S(t) \\
\beta_{(5 D) Y_{b}}^{(1)}= & Y_{b}\left(3 \operatorname{Tr}\left(Y_{b}^{\dagger} Y_{b}\right)+\operatorname{Tr}\left(Y_{\tau}^{\dagger} Y_{\tau}\right)+3 Y_{b}^{\dagger} Y_{b}+Y_{t}^{\dagger} Y_{t}+Y_{F}^{\dagger} Y_{F}\right) \pi S(t)^{2} \\
& -Y_{b}\left(\frac{25}{15} g_{1}^{2}+3 g_{2}^{2}+\frac{16}{3} g_{3}^{2}\right) S(t)
\end{aligned}
$$




$$
\begin{aligned}
\beta_{(5 D) Y_{\tau}}^{(1)}= & Y_{\tau}\left(3 \operatorname{Tr}\left(Y_{b}^{\dagger} Y_{b}\right)+\operatorname{Tr}\left(Y_{\tau}^{\dagger} Y_{\tau}\right)+3 Y_{\tau}^{\dagger} Y_{\tau}+Y_{F}^{\dagger} Y_{F}\right) \pi S(t)^{2} \\
& -Y_{\tau}\left(\frac{15}{5} g_{1}^{2}+3 g_{2}^{2}\right) S(t) \\
\beta_{(5 D) Y_{F}}^{(1)}= & Y_{F}\left(2 Y_{F}^{\dagger} Y_{F}+3 \operatorname{Tr}\left(Y_{b}^{\dagger} Y_{b}\right)+3 \operatorname{Tr}\left(Y_{t}^{\dagger} Y_{t}\right)+\operatorname{Tr}\left(Y_{\tau}^{\dagger} Y_{\tau}\right)\right) \pi S(t)^{2} \\
& -\left(3 g_{1}^{2}+3 g_{2}^{2}\right) S(t) .
\end{aligned}
$$

Note that the evolution equations for $Y_{u, c}, Y_{d, s}$ and $Y_{e, \mu}$ can be read from eq. (B.28), since the first and second generation live on the brane.

\section{B.2.6 Yukawa coupling RGEs for model 3}

The five dimensional contributions for model 3 are given by

$$
\begin{aligned}
& \beta_{(5 D) Y_{t}}^{(1)}=Y_{t}\left(\left(6 Y_{t}^{\dagger} Y_{t}+2 Y_{b}^{\dagger} Y_{b}+2 Y_{F}^{\dagger} Y_{F}\right)-\left(\frac{79}{30} g_{1}^{2}+\frac{9}{2} g_{2}^{2}+\frac{32}{3} g_{3}^{2}\right)\right) S(t) \\
& \beta_{(5 D) Y_{b}}^{(1)}=Y_{b}\left(\left(6 Y_{b}^{\dagger} Y_{b}+2 Y_{t}^{\dagger} Y_{t}+2 Y_{F}^{\dagger} Y_{F}\right)-\left(\frac{55}{30} g_{1}^{2}+\frac{9}{2} g_{2}^{2}+\frac{32}{3} g_{3}^{2}\right)\right) S(t) \\
& \beta_{(5 D) Y_{e}}^{(1)}=Y_{\tau}\left(6 Y_{\tau}^{\dagger} Y_{\tau}+2 Y_{F}^{\dagger} Y_{F}-\left(\frac{9}{2} g_{1}^{2}+\frac{9}{2} g_{2}^{2}\right)\right) S(t) \\
& \beta_{(5 D) Y_{F}}^{(1)}=Y_{F}\left(4 Y_{F}^{\dagger} Y_{F}-\left(3 g_{1}^{2}+3 g_{2}^{2}\right)\right) S(t) .
\end{aligned}
$$

\section{B.3 Trilinear soft breaking parameters}

\section{B.3.1 Trilinear soft breaking parameters for model 1}

$$
\begin{aligned}
\beta_{(5 D) T_{u}}^{(1)}= & T_{u}\left(\left(18 Y_{u}^{\dagger} Y_{u}+2 Y_{d}^{\dagger} Y_{d}+2 Y_{F}^{\dagger} Y_{F}\right)-\left(\frac{79}{30} g_{1}^{2}+\frac{9}{2} g_{2}^{2}+\frac{32}{3} g_{3}^{2}\right)\right) S(t) \\
& +Y_{u}\left(4 T_{d} Y_{d}^{\dagger}+4 Y_{F}^{\dagger} T_{F}+\frac{79}{15} g_{1}^{2} M_{1}+9 g_{2}^{2} M_{2}+\frac{64}{3} g_{3}^{2} M_{3}\right) S(t) \\
\beta_{(5 D) T_{d}}^{(1)}= & T_{d}\left(\left(18 Y_{d}^{\dagger} Y_{d}+2 Y_{u}^{\dagger} Y_{u}+2 Y_{F}^{\dagger} Y_{F}\right)-\left(\frac{55}{30} g_{1}^{2}+\frac{9}{2} g_{2}^{2}+\frac{32}{3} g_{3}^{2}\right)\right) S(t) \\
& +Y_{d}\left(4 Y_{F}^{\dagger} T_{F}+4 T_{u} Y_{u}^{\dagger}+2 T_{e} Y_{e}^{\dagger}+\frac{55}{15} g_{1}^{2} M_{1}+9 g_{2}^{2} M_{2}+\frac{64}{3} g_{3}^{2} M_{3}\right) S(t) \\
\beta_{(5 D) T_{e}}^{(1)}= & T_{e}\left(18 Y_{e}^{\dagger} Y_{e}+2 Y_{F}^{\dagger} Y_{F}-\left(\frac{9}{2} g_{1}^{2}+\frac{9}{2} g_{2}^{2}\right)\right) S(t) \\
& +Y_{e}\left(6 T_{d} Y_{d}^{\dagger}+4 Y_{F}^{\dagger} T_{F}+\frac{18}{2} g_{1}^{2} M_{1}+9 g_{2}^{2} M_{2}\right) S(t) \\
\beta_{(5 D) T_{F}}^{(1)}= & T_{F}\left(12 Y_{F}^{\dagger} Y_{F}-\left(3 g_{1}^{2}+3 g_{2}^{2}\right)\right) S(t)+Y_{F}\left(6 g_{1}^{2} M_{1}+6 g_{2}^{2} M_{2}\right) S(t)
\end{aligned}
$$




\section{B.3.2 Trilinear soft breaking parameters for model 2}

$$
\begin{aligned}
\beta_{(5 D) T_{t}}^{(1)}= & -T_{t}\left(\frac{31}{15} g_{1}^{2}+3 g_{2}^{2}+\frac{16}{3} g_{3}^{2}\right) S(t)+Y_{t}\left(\frac{62}{15} g_{1}^{2} M_{1}+6 g_{2}^{2} M_{2}+\frac{32}{3} g_{3}^{2} M_{3}\right) S(t) \\
& +Y_{t}\left(6 \operatorname{Tr}\left(Y_{t}^{\dagger} T_{t}\right)+6 Y_{t}^{\dagger} T_{t}+2 Y_{b}^{\dagger} T_{b}+2 Y_{F}^{\dagger} T_{F}\right) \pi S(t)^{2} \\
& +T_{t}\left(3 \operatorname{Tr}\left(Y_{t}^{\dagger} Y_{t}\right)+3 Y_{t}^{\dagger} Y_{t}+Y_{b}^{\dagger} Y_{b}+Y_{F}^{\dagger} Y_{F}\right) \pi S(t)^{2} \\
\beta_{(5 D) T_{b}}^{(1)}= & -T_{b}\left(\frac{25}{15} g_{1}^{2}+3 g_{2}^{2}+\frac{16}{3} g_{3}^{2}\right) S(t)+Y_{b}\left(\frac{50}{15} g_{1}^{2} M_{1}+6 g_{2}^{2} M_{2}+\frac{32}{3} g_{3}^{2} M_{3}\right) S(t) \\
& +Y_{b}\left(6 \operatorname{Tr}\left(Y_{b}^{\dagger} T_{b}\right)+2 \operatorname{Tr}\left(Y_{\tau}^{\dagger} T_{\tau}\right)+6 Y_{b}^{\dagger} T_{b}+2 Y_{t}^{\dagger} T_{t}+2 Y_{F}^{\dagger} T_{F}\right) \pi S(t)^{2} \\
& +T_{b}\left(3 \operatorname{Tr}\left(Y_{b}^{\dagger} Y_{b}\right)+\operatorname{Tr}\left(Y_{\tau}^{\dagger} Y_{\tau}\right)+3 Y_{b}^{\dagger} Y_{b}+Y_{t}^{\dagger} Y_{t}+Y_{F}^{\dagger} Y_{F}\right) \pi S(t)^{2} \\
\beta_{(5 D) T_{\tau}}^{(1)}= & -T_{\tau}\left(3 g_{1}^{2}+3 g_{2}^{2}\right) S(t)+Y_{\tau}\left(6 g_{1}^{2} M_{1}+6 g_{2}^{2} M_{2}\right) S(t) \\
& +Y_{\tau}\left(6 \operatorname{Tr}\left(Y_{b}^{\dagger} T_{b}\right)+2 \operatorname{Tr}\left(Y_{\tau}^{\dagger} T_{\tau}\right)+6 Y_{\tau}^{\dagger} T_{\tau}+2 Y_{F}^{\dagger} T_{F}\right) \pi S(t)^{2} \\
& +T_{\tau}\left(3 \operatorname{Tr}\left(Y_{b}^{\dagger} Y_{b}\right)+\operatorname{Tr}\left(Y_{\tau}^{\dagger} Y_{\tau}\right)+3 Y_{\tau}^{\dagger} Y_{\tau}+Y_{F}^{\dagger} Y_{F}\right) \pi S(t)^{2} \\
\beta_{(5 D) T_{F}}^{(1)} & -T_{F}\left(3 g_{1}^{2}+3 g_{2}^{2}\right) S(t)+Y_{F}\left(6 g_{1}^{2} M_{1}+6 g_{2}^{2} M_{2}\right) S(t) \\
& +Y_{F}\left(6 \operatorname{Tr}\left(Y_{b}^{\dagger} T_{b}\right)+6 \operatorname{Tr}\left(Y_{t}^{\dagger} T_{t}\right)+2 \operatorname{Tr}\left(Y_{\tau}^{\dagger} T_{\tau}\right)+4 Y_{F}^{\dagger} T_{F}\right) \pi S(t)^{2} \\
& +T_{F}\left(3 \operatorname{Tr}\left(Y_{b}^{\dagger} Y_{b}\right)+3 \operatorname{Tr}\left(Y_{t}^{\dagger} Y_{t}\right)+\operatorname{Tr}\left(Y_{\tau}^{\dagger} Y_{\tau}\right)+2 Y_{F}^{\dagger} Y_{F}\right) \pi S(t)^{2} .
\end{aligned}
$$

\section{B.4 Soft mass parameters}

\section{B.4.1 Gaugino soft mass parameters}

The gaugino soft masses in 5D run following

$$
\beta_{M_{i}}^{(1)}=2 b^{i} g_{i}^{2} M_{i}+2(S(t)-1) b_{5 D}^{i} g_{i}^{2} M_{i} .
$$

\section{B.4.2 Scalar soft mass parameters for model 1}

$$
\begin{aligned}
\beta_{m_{q}^{2}}^{(1)}= & \left(-\frac{8}{15} g_{1}^{2} \mathbf{1}\left|M_{1}\right|^{2}-\frac{64}{3} g_{3}^{2} \mathbf{1}\left|M_{3}\right|^{2}-12 g_{2}^{2} \mathbf{1}\left|M_{2}\right|^{2}+4 m_{H_{d}}^{2} Y_{d}^{\dagger} Y_{d}+4 m_{H_{u}}^{2} Y_{u}^{\dagger} Y_{u}\right) S(t) \\
& +\left(4 T_{d}^{\dagger} T_{d}+4 T_{u}^{\dagger} T_{u}+2 m_{q}^{2} Y_{d}^{\dagger} Y_{d}+2 m_{q}^{2} Y_{u}^{\dagger} Y_{u}+4 Y_{d}^{\dagger} m_{d}^{2} Y_{d}+2 Y_{d}^{\dagger} Y_{d} m_{q}^{2}\right) S(t) \\
& +\left(4 Y_{u}^{\dagger} m_{u}^{2} Y_{u}+2 Y_{u}^{\dagger} Y_{u} m_{q}^{2}+\frac{2}{\sqrt{15}} g_{1} \mathbf{1} \sigma_{1,1}\right) S(t) \\
\beta_{m_{u}^{2}}^{(1)}= & \left(-\frac{64}{15} g_{1}^{2} \mathbf{1}\left|M_{1}\right|^{2}-\frac{64}{3} g_{3}^{2} \mathbf{1}\left|M_{3}\right|^{2}+8 m_{H_{u}}^{2} Y_{u} Y_{u}^{\dagger}+8 T_{u} T_{u}^{\dagger}+4 m_{u}^{2} Y_{u} Y_{u}^{\dagger}\right) S(t) \\
& +\left(8 Y_{u} m_{q}^{2} Y_{u}^{\dagger}+4 Y_{u} Y_{u}^{\dagger} m_{u}^{2}-4 \sqrt{\frac{2}{15}} g_{1} \mathbf{1} \sigma_{1,1}\right) S(t)
\end{aligned}
$$




$$
\begin{aligned}
\beta_{m_{d}^{2}}^{(1)}= & \left(-\frac{16}{15} g_{1}^{2} \mathbf{1}\left|M_{1}\right|^{2}-\frac{64}{3} g_{3}^{2} \mathbf{1}\left|M_{3}\right|^{2}+8 m_{H_{d}}^{2} Y_{d} Y_{d}^{\dagger}+8 T_{d} T_{d}^{\dagger}+4 m_{d}^{2} Y_{d} Y_{d}^{\dagger}\right) S(t) \\
& +\left(8 Y_{d} m_{q}^{2} Y_{d}^{\dagger}+4 Y_{d} Y_{d}^{\dagger} m_{d}^{2}+2 \sqrt{\frac{2}{15}} g_{1} \mathbf{1} \sigma_{1,1}\right) S(t) \\
\beta_{m_{l}^{2}}^{(1)}= & \left(-\frac{12}{5} g_{1}^{2} \mathbf{1}\left|M_{1}\right|^{2}-12 g_{2}^{2} \mathbf{1}\left|M_{2}\right|^{2}+4 m_{H_{d}}^{2} Y_{e}^{\dagger} Y_{e}+4 T_{e}^{\dagger} T_{e}+2 m_{l}^{2} Y_{e}^{\dagger} Y_{e}\right) S(t) \\
& +\left(4 Y_{e}^{\dagger} m_{e}^{2} Y_{e}+2 Y_{e}^{\dagger} Y_{e} m_{l}^{2}-\sqrt{\frac{6}{5}} g_{1} \mathbf{1} \sigma_{1,1}\right) S(t) \\
\beta_{m_{e}^{2}}^{(1)}= & 2\left(4 m_{H_{d}}^{2} Y_{e} Y_{e}^{\dagger}+4 T_{e} T_{e}^{\dagger}+4 Y_{e} m_{l}^{2} Y_{e}^{\dagger}+2 m_{e}^{2} Y_{e} Y_{e}^{\dagger}+2 Y_{e} Y_{e}^{\dagger} m_{e}^{2}\right) S(t) \\
& +\left(2 \sqrt{\frac{6}{5}} g_{1} \mathbf{1} \sigma_{1,1}-\frac{48}{5} g_{1}^{2} \mathbf{1}\left|M_{1}\right|^{2}\right) S(t)
\end{aligned}
$$

In model 1 the two Higgs doublet soft masses obey the RGE's

$$
\begin{aligned}
\beta_{m_{H_{d}}^{2}}^{(1)}= & \left(-\frac{6}{5} g_{1}^{2}\left|M_{1}\right|^{2}-6 g_{2}^{2}\left|M_{2}\right|^{2}-\sqrt{\frac{3}{5}} g_{1} \sigma_{1,1}+6 m_{H_{d}}^{2} \operatorname{Tr}\left(Y_{d} Y_{d}^{\dagger}\right)\right) S(t) \\
& +\left(2 m_{H_{d}}^{2} \operatorname{Tr}\left(Y_{e} Y_{e}^{\dagger}\right)+6 \operatorname{Tr}\left(T_{d}^{*} T_{d}^{T}\right)+2 \operatorname{Tr}\left(T_{e}^{*} T_{e}^{T}\right)+6 \operatorname{Tr}\left(m_{d}^{2} Y_{d} Y_{d}^{\dagger}\right)\right) S(t) \\
& +\left(2 \operatorname{Tr}\left(m_{e}^{2} Y_{e} Y_{e}^{\dagger}\right)+2 \operatorname{Tr}\left(m_{l}^{2} Y_{e}^{\dagger} Y_{e}\right)+6 \operatorname{Tr}\left(m_{q}^{2} Y_{d}^{\dagger} Y_{d}\right)\right) S(t) \\
\beta_{m_{H_{u}}^{2}}^{(1)}= & \left(-\frac{6}{5} g_{1}^{2}\left|M_{1}\right|^{2}-6 g_{2}^{2}\left|M_{2}\right|^{2}+\sqrt{\frac{3}{5}} g_{1} \sigma_{1,1}+6 m_{H_{u}}^{2} \operatorname{Tr}\left(Y_{u} Y_{u}^{\dagger}\right)\right) S(t) \\
& +\left(6 \operatorname{Tr}\left(T_{u}^{*} T_{u}^{T}\right)+6 \operatorname{Tr}\left(m_{q}^{2} Y_{u}^{\dagger} Y_{u}\right)+6 \operatorname{Tr}\left(m_{u}^{2} Y_{u} Y_{u}^{\dagger}\right)\right) S(t) \\
\beta_{m_{F^{ \pm}}^{(1)}=} & \left(-\frac{24}{5} g_{1}^{2}\left|M_{1}\right|^{2}+2 m_{H_{u, d}}^{2} Y_{F}^{\dagger} Y_{F}+2 T_{F}^{\dagger} T_{F}+m_{F^{ \pm}}^{2} Y_{F}^{\dagger} Y_{F}\right) S(t) \\
& +\left(Y_{F}^{\dagger} Y_{F} m_{F^{ \pm}}^{2}\right) S(t)
\end{aligned}
$$

\section{B.4.3 Scalar soft mass parameters for model 2}

$$
\begin{aligned}
\beta_{m_{q_{3}}^{2}}^{(1)}= & \left(-\frac{2}{15} g_{1}^{2} \mathbf{1}\left|M_{1}\right|^{2}-\frac{32}{3} g_{3}^{2} \mathbf{1}\left|M_{3}\right|^{2}-6 g_{2}^{2} \mathbf{1}\left|M_{2}\right|^{2}\right) S(t)+\left(2 m_{H_{d}}^{2} Y_{b}^{\dagger} Y_{b}+2 m_{H_{u}}^{2} Y_{t}^{\dagger} Y_{t}\right) \pi S(t)^{2} \\
& +\left(2 T_{b}^{\dagger} T_{b}+2 T_{t}^{\dagger} T_{t}+m_{q_{3}}^{2} Y_{b}^{\dagger} Y_{b}+m_{q_{3}}^{2} Y_{t}^{\dagger} Y_{t}+2 Y_{b}^{\dagger} m_{d_{3}}^{2} Y_{b}+Y_{b}^{\dagger} Y_{b} m_{q_{3}}^{2}\right) \pi S(t)^{2} \\
& +\left(2 Y_{t}^{\dagger} m_{u_{3}}^{2} Y_{t}+Y_{t}^{\dagger} Y_{t} m_{q_{3}}^{2}\right) \pi S(t)^{2}+\left(\frac{1}{\sqrt{15}} g_{1} \mathbf{1} \sigma_{1,1}\right) S(t) \\
\beta_{m_{u_{3}}^{2}}^{(1)}= & \left(-\frac{32}{15} g_{1}^{2} \mathbf{1}\left|M_{1}\right|^{2}-\frac{32}{3} g_{3}^{2} \mathbf{1}\left|M_{3}\right|^{2}\right) S(t)+\left(4 m_{H_{u}}^{2} Y_{t} Y_{t}^{\dagger}+4 T_{t} T_{t}^{\dagger}+2 m_{u_{3}}^{2} Y_{t} Y_{t}^{\dagger}\right) \pi S(t)^{2} \\
& +\left(4 Y_{t} m_{q_{3}}^{2} Y_{t}^{\dagger}+2 Y_{t} Y_{t}^{\dagger} m_{u_{3}}^{2}\right) \pi S(t)^{2}-\left(4 \frac{1}{\sqrt{15}} g_{1} \mathbf{1} \sigma_{1,1}\right) S(t)
\end{aligned}
$$




$$
\begin{aligned}
\beta_{m_{d_{3}}^{2}}^{(1)}= & \left(-\frac{8}{15} g_{1}^{2} \mathbf{1}\left|M_{1}\right|^{2}-\frac{32}{3} g_{3}^{2} \mathbf{1}\left|M_{3}\right|^{2}\right) S(t)+\left(4 m_{H_{d}}^{2} Y_{b} Y_{b}^{\dagger}+4 T_{b} T_{b}^{\dagger}+2 m_{d_{3}}^{2} Y_{b} Y_{b}^{\dagger}\right) \pi S(t)^{2} \\
& +\left(4 Y_{b} m_{q_{3}}^{2} Y_{b}^{\dagger}+2 Y_{b} Y_{b}^{\dagger} m_{d_{3}}^{2}\right) \pi S(t)^{2}+\left(2 \sqrt{\frac{1}{15}} g_{1} \mathbf{1} \sigma_{1,1}\right) S(t) \\
\beta_{m_{l_{3}}^{2}}^{(1)}= & \left(-\frac{6}{5} g_{1}^{2} \mathbf{1}\left|M_{1}\right|^{2}-6 g_{2}^{2} \mathbf{1}\left|M_{2}\right|^{2}\right) S(t)+\left(4 m_{H_{d}}^{2} Y_{\tau}^{\dagger} Y_{\tau}+4 T_{\tau}^{\dagger} T_{\tau}+2 m_{l_{3}}^{2} Y_{\tau}^{\dagger} Y_{\tau}\right) \pi S(t)^{2} \\
& +\left(2 Y_{\tau}^{\dagger} m_{e_{3}}^{2} Y_{\tau}+Y_{\tau}^{\dagger} Y_{\tau} m_{l_{3}}^{2}\right) \pi S(t)^{2}-\left(\sqrt{\frac{3}{5}} g_{1} \mathbf{1} \sigma_{1,1}\right) S(t) \\
\beta_{m_{e_{3}}^{2}}^{(1)}= & 2\left(2 m_{H_{d}}^{2} Y_{\tau} Y_{\tau}^{\dagger}+2 T_{\tau} T_{\tau}^{\dagger}+2 Y_{\tau} m_{l_{3}}^{2} Y_{\tau}^{\dagger}+m_{e_{3}}^{2} Y_{\tau} Y_{\tau}^{\dagger}+Y_{\tau} Y_{\tau}^{\dagger} m_{e_{3}}^{2}\right) \pi S(t)^{2} \\
& +\left(2 \sqrt{\frac{3}{5}} g_{1} \mathbf{1} \sigma_{1,1}-\frac{24}{5} g_{1}^{2} \mathbf{1}\left|M_{1}\right|^{2}\right) S(t)
\end{aligned}
$$

In model 2 the two Higgs doublet soft masses obey the RGE's

$$
\begin{aligned}
\beta_{m_{H_{d}}^{2}}^{(1)}= & \left(-\frac{6}{5} g_{1}^{2}\left|M_{1}\right|^{2}-6 g_{2}^{2}\left|M_{2}\right|^{2}-\sqrt{\frac{3}{5}} g_{1} \sigma_{1,1}\right) S(t)+\left(6 m_{H_{d}}^{2} \operatorname{Tr}\left(Y_{b} Y_{b}^{\dagger}\right)\right) \pi S(t)^{2} \\
& +\left(2 m_{H_{d}}^{2} \operatorname{Tr}\left(Y_{\tau} Y_{\tau}^{\dagger}\right)+6 \operatorname{Tr}\left(T_{b}^{*} T_{b}^{T}\right)+2 \operatorname{Tr}\left(T_{\tau}^{*} T_{\tau}^{T}\right)+6 \operatorname{Tr}\left(m_{d_{3}}^{2} Y_{b} Y_{b}^{\dagger}\right)\right) \pi S(t)^{2} \\
& +\left(2 \operatorname{Tr}\left(m_{e_{3}}^{2} Y_{\tau} Y_{\tau}^{\dagger}\right)+2 \operatorname{Tr}\left(m_{l_{3}}^{2} Y_{\tau}^{\dagger} Y_{\tau}\right)+6 \operatorname{Tr}\left(m_{q_{3}}^{2} Y_{b}^{\dagger} Y_{b}\right)\right) \pi S(t)^{2} \\
\beta_{m_{H_{u}}^{2}}^{(1)}= & \left(-\frac{6}{5} g_{1}^{2}\left|M_{1}\right|^{2}-6 g_{2}^{2}\left|M_{2}\right|^{2}+\sqrt{\frac{3}{5}} g_{1} \sigma_{1,1}\right) S(t)+\left(6 m_{H_{u}}^{2} \operatorname{Tr}\left(Y_{t} Y_{t}^{\dagger}\right)\right) \pi S(t)^{2} \\
& +\left(6 \operatorname{Tr}\left(T_{t}^{*} T_{t}^{T}\right)+6 \operatorname{Tr}\left(m_{q_{3}}^{2} Y_{t}^{\dagger} Y_{t}\right)+6 \operatorname{Tr}\left(m_{u_{3}}^{2} Y_{t} Y_{t}^{\dagger}\right)\right) \pi S(t)^{2} \\
\beta_{m_{F^{ \pm}}^{2}}^{(1)}= & -\frac{24}{5} g_{1}^{2}\left|M_{1}\right|^{2} S(t)+\left(2 m_{H_{u, d}}^{2} Y_{F}^{\dagger} Y_{F}+2 T_{F}^{\dagger} T_{F}+m_{F^{ \pm}}^{2} Y_{F}^{\dagger} Y_{F}\right) \pi S(t)^{2} \\
& +\left(Y_{F}^{\dagger} Y_{F} m_{F^{ \pm}}^{2}\right) \pi S(t)^{2}
\end{aligned}
$$

\section{B.5 Bilinear parameters $\boldsymbol{\mu}$ and $\boldsymbol{B}_{\boldsymbol{\mu}}$}

In $5 \mathrm{D}$ these are given by:

$$
\begin{aligned}
\beta_{\mu}^{(1)}= & \mu\left(3 \operatorname{Tr}\left(Y_{u}^{\dagger} Y_{u}\right)+3 \operatorname{Tr}\left(Y_{d}^{\dagger} Y_{d}\right)+\operatorname{Tr}\left(Y_{e}^{\dagger} Y_{e}\right)-\frac{3}{5} g_{1}^{2}-3 g_{2}^{2}\right) S(t) \\
\beta_{\dot{\mu}}^{(1)}= & \left(2 \mu\left(Y_{F} Y_{F}^{\dagger}\right)-\frac{12}{5} \mu g_{1}^{2}\right) S(t) \\
\beta_{B_{\mu}}^{(1)}= & B_{\mu}\left(-3 g_{2}^{2}-\frac{3}{5} g_{1}^{2}+3 \operatorname{Tr}\left(Y_{u}^{\dagger} Y_{u}\right)+3 \operatorname{Tr}\left(Y_{d}^{\dagger} Y_{d}\right)+\operatorname{Tr}\left(Y_{e}^{\dagger} Y_{e}\right)\right) S(t) \\
& +\mu\left(6 g_{2}^{2} M_{2}+\frac{6}{5} g_{1}^{2} M_{1}+6 \operatorname{Tr}\left(Y_{u}^{\dagger} T_{u}\right)+6 \operatorname{Tr}\left(Y_{d}^{\dagger} T_{d}\right)+2 \operatorname{Tr}\left(Y_{e}^{\dagger} T_{e}\right)\right) S(t) \\
\beta_{B_{\dot{\mu}}}^{(1)}= & \left(-\frac{12}{5} B_{\dot{\mu}} g_{1}^{2}+\frac{24}{5} \mu g_{1}^{2} M_{1}+2 B_{\dot{\mu}} Y_{F}^{\dagger} Y_{F}+4 \hat{\mu} Y_{F}^{\dagger} Y_{F}\right) S(t) .
\end{aligned}
$$


Open Access. This article is distributed under the terms of the Creative Commons Attribution License (CC-BY 4.0), which permits any use, distribution and reproduction in any medium, provided the original author(s) and source are credited.

\section{References}

[1] ATLAS collaboration, Observation of a new particle in the search for the Standard Model Higgs boson with the ATLAS detector at the LHC, Phys. Lett. B 716 (2012) 1 [arXiv: 1207.7214] [INSPIRE].

[2] CMS collaboration, Observation of a new boson at a mass of $125 \mathrm{GeV}$ with the CMS experiment at the LHC, Phys. Lett. B 716 (2012) 30 [arXiv:1207.7235] [INSPIRE].

[3] P. Batra, A. Delgado, D.E. Kaplan and T.M.P. Tait, The Higgs mass bound in gauge extensions of the minimal supersymmetric standard model, JHEP 02 (2004) 043 [hep-ph/0309149] [INSPIRE].

[4] A. Maloney, A. Pierce and J.G. Wacker, D-terms, unification and the Higgs mass, JHEP 06 (2006) 034 [hep-ph/0409127] [INSPIRE].

[5] A. Bharucha, A. Goudelis and M. McGarrie, En-gauging Naturalness, Eur. Phys. J. C 74 (2014) 2858 [arXiv: 1310.4500] [INSPIRE].

[6] M. McGarrie, G. Moortgat-Pick and S. Porto, Confronting Higgs couplings from D-term extensions and Natural SUSY at the LHC and ILC, Eur. Phys. J. C 75 (2015) 150 [arXiv: 1411.2040] [INSPIRE].

[7] ATLAS collaboration, Search for pair-produced top squarks decaying into a charm quark and the lightest neutralinos with $20.3 \mathrm{fb}^{-1}$ of pp collisions at $\sqrt{\mathrm{s}}=8 \mathrm{TeV}$ with the ATLAS detector at the LHC, ATLAS-CONF-2013-068 (2013) [INSPIRE].

[8] ATLAS collaboration, Searches for direct scalar top pair production in final states with two leptons using the stransverse mass variable and a multivariate analysis technique in $\sqrt{s}=8 \mathrm{TeV}$ pp collisions using 20.3 fb $\mathrm{fb}^{-1}$ of ATLAS data, ATLAS-CONF-2013-065 (2013) [INSPIRE].

[9] ATLAS collaboration, Search for strong production of supersymmetric particles in final states with missing transverse momentum and at least three b-jets using $20.1 \mathrm{fb}^{-1}$ of pp collisions at $\sqrt{s}=8 \mathrm{TeV}$ with the ATLAS Detector, ATLAS-CONF-2013-061 (2013) [INSPIRE].

[10] ATLAS collaboration, Search for direct top squark pair production in final states with two leptons in $\sqrt{s}=8 \mathrm{TeV}$ pp collisions using $20 \mathrm{fb}^{-1}$ of ATLAS data, ATLAS-CONF-2013-048 (2013) [INSPIRE].

[11] ATLAS collaboration, Search for direct top squark pair production in final states with one isolated lepton, jets and missing transverse momentum in sqrts $=8 \mathrm{TeV}$ pp collisions using $21 \mathrm{fb}^{-1}$ of ATLAS data, ATLAS-CONF-2013-037 (2013) [INSPIRE].

[12] ATLAS collaboration, Search for direct stop pair production in events with a $Z$ boson, b-jets and missing transverse energy with the ATLAS detector using $21 \mathrm{fb}^{-1}$ from proton-proton collision at $\sqrt{s}=8 \mathrm{TeV}$, ATLAS-CONF-2013-025 (2013) [INSPIRE].

[13] ATLAS collaboration, Search for direct production of the top squark in the all-hadronic $t \bar{t}+E_{T}^{\text {miss }}$ final state in $21 \mathrm{fb}^{-1}$ of $p$-p collisions at $\sqrt{s}=8 \mathrm{TeV}$ with the ATLAS detector, ATLAS-CONF-2013-024 (2013) [INSPIRE]. 
[14] CMS collaboration, Search for top-squark pair production in the single-lepton final state in pp collisions at $\sqrt{s}=8, T e V$, Eur. Phys. J. C 73 (2013) 2677 [arXiv:1308.1586] [InSPIRE].

[15] CMS collaboration, Search for SUSY Partners of Top and Higgs Using Diphoton Higgs Decays, CMS-PAS-SUS-13-014 (2013) [INSPIRE].

[16] CMS collaboration, Search for supersymmetry in pp collisions at $\sqrt{s}=8$ TeV in events with three leptons and at least one b-tagged jet, CMS-PAS-SUS-13-008 (2013) [INSPIRE].

[17] CMS collaboration, Search for direct top squark pair production in events with a single isolated lepton, jets and missing transverse energy at $\sqrt{s}=8 \mathrm{Te}$, CMS-PAS-SUS-12-023 (2012) [INSPIRE].

[18] A. Abdalgabar, A.S. Cornell, A. Deandrea and M. McGarrie, Large $A_{t}$ Without the Desert, JHEP 07 (2014) 158 [arXiv: 1405.1038] [INSPIRE].

[19] J.F. Oliver, J. Papavassiliou and A. Santamaria, Can power corrections be reliably computed in models with extra dimensions?, Phys. Rev. D 67 (2003) 125004 [hep-ph/0302083] [INSPIRE].

[20] M. Masip, Unification through extra dimensions at two loops, Phys. Rev. D 62 (2000) 065011 [hep-ph/0001115] [INSPIRE].

[21] S. Hossenfelder, Running coupling with minimal length, Phys. Rev. D 70 (2004) 105003 [hep-ph/0405127] [INSPIRE].

[22] A. Hebecker, 5-D super Yang-Mills theory in 4-D superspace, superfield brane operators and applications to orbifold GUTs, Nucl. Phys. B 632 (2002) 101 [hep-ph/0112230] [InSPIRE].

[23] E.A. Mirabelli and M.E. Peskin, Transmission of supersymmetry breaking from a four-dimensional boundary, Phys. Rev. D 58 (1998) 065002 [hep-th/9712214] [INSPIRE].

[24] M. McGarrie and R. Russo, General Gauge Mediation in 5D, Phys. Rev. D 82 (2010) 035001 [arXiv: 1004.3305] [INSPIRE].

[25] A.S. Cornell, A. Deandrea, L.-X. Liu and A. Tarhini, Scaling of the CKM Matrix in the 5D MSSM, Phys. Rev. D 85 (2012) 056001 [arXiv:1110.1942] [INSPIRE].

[26] M. McGarrie, 5D Maximally Supersymmetric Yang-Mills in 4D Superspace: Applications, JHEP 04 (2013) 161 [arXiv: 1303.4534] [INSPIRE].

[27] A. Delgado, A. Pomarol and M. Quirós, Supersymmetry and electroweak breaking from extra dimensions at the TeV scale, Phys. Rev. D 60 (1999) 095008 [hep-ph/9812489] [INSPIRE].

[28] K.R. Dienes, E. Dudas and T. Gherghetta, Extra space-time dimensions and unification, Phys. Lett. B 436 (1998) 55 [hep-ph/9803466] [INSPIRE].

[29] K.R. Dienes, E. Dudas and T. Gherghetta, Grand unification at intermediate mass scales through extra dimensions, Nucl. Phys. B 537 (1999) 47 [hep-ph/9806292] [INSPIRE].

[30] I. Aitchison, Supersymmetry in Particle Physics. An Elementary Introduction, Cambridge University Press (2007).

[31] R.N. Mohapatra, Supersymmetric grand unification, hep-ph/9801235 [INSPIRE].

[32] M. Kakizaki, Proton stability in low-scale extra-dimensional grand unified theories, Phys. Rev. D 88 (2013) 095017 [arXiv:1307.0535] [INSPIRE].

[33] S.P. Martin, A Supersymmetry primer, Adv. Ser.Direct. High Energy Phys. 21 (2010) 1 [Adv. Ser. Direct. High Energy Phys. 18 (1998) 1] [hep-ph/9709356] [INSPIRE]. 
[34] M. Drees, R. Godbole and P. Roy, Theory and phenomenology of sparticles: An account of four-dimensional $N=1$ supersymmetry in high energy physics, 1st edition, World Scientific (2004).

[35] G. Bhattacharyya, A. Datta, S.K. Majee and A. Raychaudhuri, Power law scaling in universal extra dimension scenarios, Nucl. Phys. B 760 (2007) 117 [hep-ph/0608208] [INSPIRE].

[36] A. Abdalgabar and A.S. Cornell, Evolution of Yukawa Couplings and Quark Flavour Mixings in the 5D MSSM, J. Phys. Conf. Ser. 455 (2013) 012050 [arXiv:1305.3729] [INSPIRE].

[37] G. Bhattacharyya and T.S. Ray, A phenomenological study of $5 d$ supersymmetry, JHEP 05 (2010) 040 [arXiv:1003.1276] [INSPIRE].

[38] K. Benakli, M. Goodsell, F. Staub and W. Porod, Constrained minimal Dirac gaugino supersymmetric standard model, Phys. Rev. D 90 (2014) 045017 [arXiv:1403.5122] [INSPIRE].

[39] F. Paccetti Correia, M.G. Schmidt and Z. Tavartkiladze, Gauge coupling unification and phenomenology of selected orbifold 5-D N =1 SUSY models, Nucl. Phys. B 649 (2003) 39 [hep-ph/0204080] [INSPIRE].

[40] ATLAS collaboration, Search for squarks and gluinos with the ATLAS detector in final states with jets and missing transverse momentum and $20.3 \mathrm{fb}^{-1}$ of $\sqrt{\mathrm{s}}=8 \mathrm{TeV}$ proton-proton collision data, ATLAS-CONF-2013-047 (2013) [INSPIRE].

[41] CMS collaboration, Search for new physics in the multijet and missing transverse momentum final state in proton-proton collisions at $\sqrt{s}=7$ TeV, Phys. Rev. Lett. 109 (2012) 171803 [arXiv: 1207.1898 ] [INSPIRE].

[42] F. Staub, From Superpotential to Model Files for FeynArts and CalcHep/CompHEP, Comput. Phys. Commun. 181 (2010) 1077 [arXiv:0909.2863] [inSPIRE].

[43] F. Staub, Automatic Calculation of supersymmetric Renormalization Group Equations and Self Energies, Comput. Phys. Commun. 182 (2011) 808 [arXiv: 1002.0840] [INSPIRE].

[44] F. Staub, SARAH 3.2: Dirac Gauginos, UFO output and more, Comput. Phys. Commun. 184 (2013) 1792 [arXiv: 1207.0906] [INSPIRE].

[45] F. Staub, SARAH 4: A tool for (not only SUSY) model builders, Comput. Phys. Commun. 185 (2014) 1773 [arXiv: 1309.7223] [INSPIRE].

[46] ATLAS collaboration, Search for new phenomena in final states with large jet multiplicities and missing transverse momentum at $\sqrt{s}=8 \mathrm{TeV}$ proton-proton collisions using the ATLAS experiment, JHEP 10 (2013) 130 [Erratum ibid. 01 (2014) 109] [arXiv:1308.1841] [INSPIRE].

[47] ATLAS collaboration, Search for strongly produced supersymmetric particles in decays with two leptons at $\sqrt{s}=8 \mathrm{TeV}$, ATLAS-CONF-2013-089 (2013) [INSPIRE].

[48] ATLAS collaboration, Search for squarks and gluinos in events with isolated leptons, jets and missing transverse momentum at $\sqrt{s}=8 \mathrm{TeV}$ with the ATLAS detector, ATLAS-CONF-2013-062 (2013) [INSPIRE].

[49] ATLAS collaboration, Search for squarks and gluinos with the ATLAS detector in final states with jets and missing transverse momentum and $20.3 \mathrm{fb}^{-1}$ of $\sqrt{s}=8 \mathrm{TeV}$ proton-proton collision data, ATLAS-CONF-2013-047 (2013) [INSPIRE]. 
[50] CMS collaboration, Search for supersymmetry in hadronic final states with missing transverse energy using the variables $\alpha_{T}$ and b-quark multiplicity in pp collisions at $\sqrt{s}=8$ TeV, Eur. Phys. J. C 73 (2013) 2568 [arXiv:1303.2985] [InSPIRE].

[51] CMS collaboration, Search for new physics in events with same-sign dileptons and jets in pp collisions at $8 \mathrm{TeV}$, CMS-PAS-SUS-13-013 (2013) [INSPIRE].

[52] CMS collaboration, Search for New Physics in the Multijets and Missing Momentum Final State in Proton-Proton Collisions at 8 TeV, CMS-PAS-SUS-13-012 (2013) [INSPIRE].

[53] CMS collaboration, Search for supersymmetry using razor variables in events with b-jets in pp collisions at $8 \mathrm{TeV}$, CMS-PAS-SUS-13-004 (2013) [INSPIRE].

[54] F. Brümmer, M. McGarrie and A. Weiler, Light third-generation squarks from flavour gauge messengers, JHEP 04 (2014) 078 [arXiv: 1312.0935] [INSPIRE].

[55] S. Abel and M. McGarrie, Natural supersymmetry and dynamical flavour with meta-stable vacua, JHEP 07 (2014) 145 [arXiv:1404.1318] [INSPIRE].

[56] M. McGarrie, Gauge Mediated Supersymmetry Breaking in Five Dimensions, arXiv:1109.6245 [INSPIRE].

[57] S. Heinemeyer, W. Hollik and G. Weiglein, FeynHiggs: A Program for the calculation of the masses of the neutral CP even Higgs bosons in the MSSM, Comput. Phys. Commun. 124 (2000) 76 [hep-ph/9812320] [INSPIRE].

[58] T. Hahn, S. Heinemeyer, W. Hollik, H. Rzehak and G. Weiglein, FeynHiggs: A program for the calculation of MSSM Higgs-boson observables - Version 2.6.5, Comput. Phys. Commun. 180 (2009) 1426 [INSPIRE].

[59] J.R. Ellis, G. Ridolfi and F. Zwirner, On radiative corrections to supersymmetric Higgs boson masses and their implications for LEP searches, Phys. Lett. B 262 (1991) 477 [INSPIRE].

[60] J.L. Lopez and D.V. Nanopoulos, New theoretical lower bounds on the Higgs sector of minimal SUSY, Phys. Lett. B 266 (1991) 397 [INSPIRE].

[61] M.S. Carena, J.R. Espinosa, M. Quirós and C.E.M. Wagner, Analytical expressions for radiatively corrected Higgs masses and couplings in the MSSM, Phys. Lett. B 355 (1995) 209 [hep-ph/9504316] [INSPIRE].

[62] H.E. Haber, R. Hempfling and A.H. Hoang, Approximating the radiatively corrected Higgs mass in the minimal supersymmetric model, Z. Phys. C 75 (1997) 539 [hep-ph/9609331] [INSPIRE].

[63] G. Degrassi, S. Heinemeyer, W. Hollik, P. Slavich and G. Weiglein, Towards high precision predictions for the MSSM Higgs sector, Eur. Phys. J. C 28 (2003) 133 [hep-ph/0212020] [INSPIRE]. 